\title{
Implementing BetterBack - a Best Practice Physiotherapy Healthcare Model for Low Back Pain Clinician and Patient Evaluation
}

\author{
Karin Schröder
}

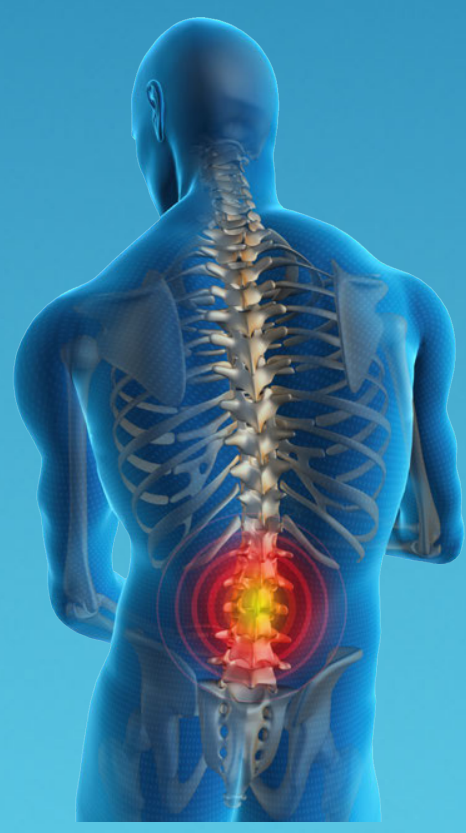





\title{
Implementing BetterBack - a Best Practice Physiotherapy Healthcare Model for Low Back Pain
}

Clinician and Patient Evaluation

\author{
Karin Schröder
}

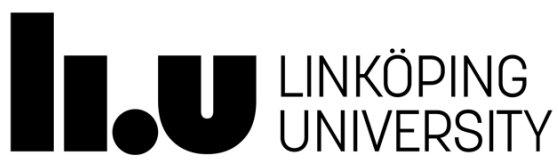

Department of Health, Medicine and Caring Sciences

Linköping University, Sweden

Linköping 2021 
(c) EY-NC This work is licensed under a Creative Commons AttributionNonCommercial 4.0 International License.

https://creativecommons.org/licenses/by-nc/4.0/

@ Karin Schröder, 2021

Pictures on the back cover: Emma Busk Winquist

Published articles have been reprinted with the permission of the copyright holder.

Printed in Sweden by LiU-Tryck, Linköping, Sweden, 2021

ISBN 978-91-7929-639-1

ISSN 0345-0082 
"All progress takes place outside the comfort zone"

Michael John Bobak 



\section{CONTENTS}

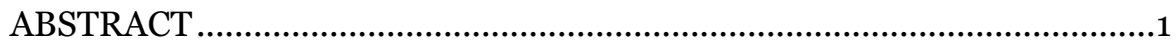

SVENSK SAMMANFATTNING …………………................................ 3

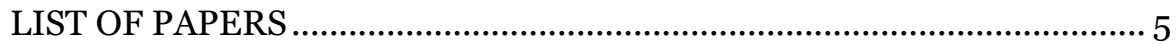

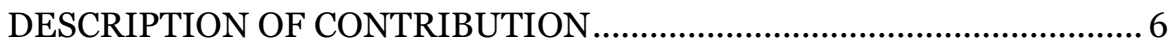

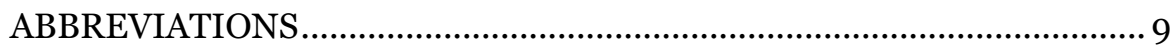

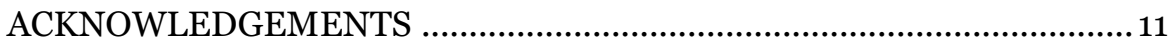

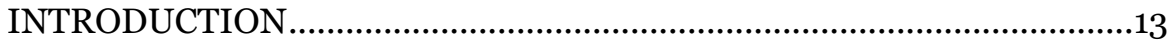

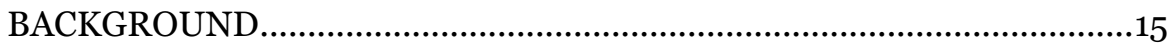

Low back pain .........................................................................................15

Prevalence and impact on health and welfare ……………………........ 15

Pathogenesis, risk, and prognosis....................................................... 16

Evidence-based practice (EBP)............................................................17

Clinical practice guidelines $(C P G)$....................................................... 17

Barriers in CPG implementation ...................................................... 19

Low back pain CPG implementation in primary care physiotherapy..... 20

Implementation Science ......................................................................... 21

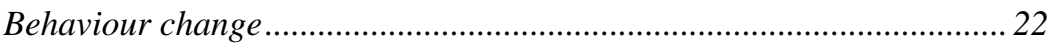

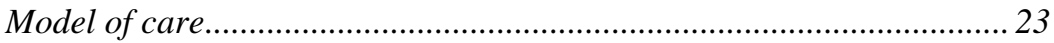

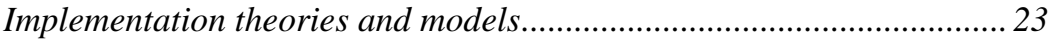

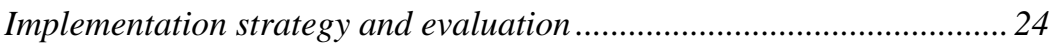

Measurement properties in questionnaire development ......................... 25

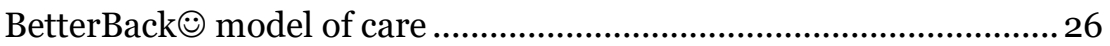

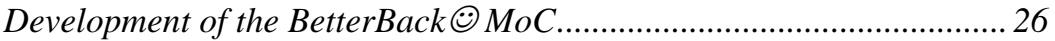

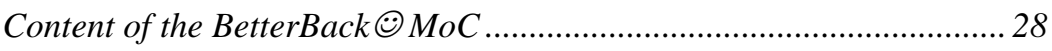

Rationale of the thesis ............................................................................31

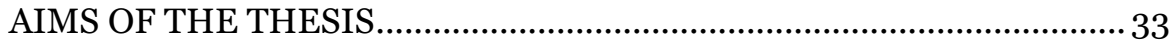

Overall aim ......................................................................................... 33 


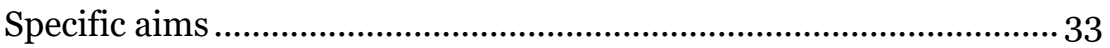

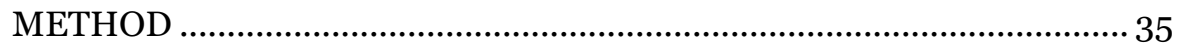

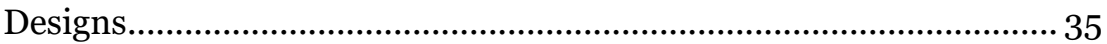

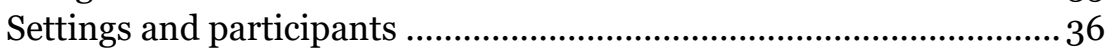

Inclusion criteria for expert panel and clinicians ................................... 37

Inclusion criteria for patients................................................................ 38

Measurements ......................................................................................... 39

Evaluation of the implementation intervention.................................... 39

Practitioner Self-Confidence Scale (PCS) ............................................. 39

Pain Attitudes and Beliefs Scale for Physiotherapists (PABS-PT) .......... 39

Determinants of Implementation Behaviour Questionnaire $(D I B Q)$...... 40

Clinical Reasoning and Process Evaluation tool (CRPE)...................... 40

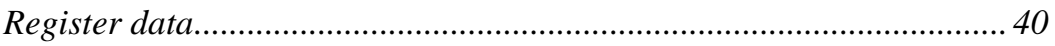

Evaluation of the clinical intervention .................................................40

STarT Back Screening Tool (SBT) ...................................................... 40

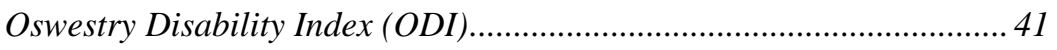

Numeric Rating Scale (NRS-LBP)...................................................... 41

European Quality of Life Questionnaire (EQ-5D).................................. 41

Brief Illness Perception Questionnaire (BIPQ) ..................................... 41

Patient Enablement Instrument (PEI) ................................................... 41

Patient Satisfaction (PS) ................................................................ 42

Patient Global Impression of Change (PGIC) ........................................ 42

Intervention ...................................................................................... 43

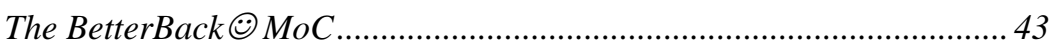

Data analyses and methodological considerations ............................... 45

Questionnaire development (Study A, Paper I)....................................... 45

Confidence, attitudes, beliefs, and implementation behaviours (Study B,

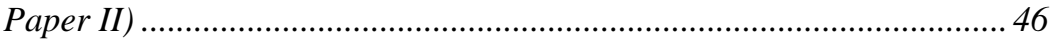

Adherence to clinical practice guidelines (Study B, Paper III) ............... 47

Patient-reported outcomes (Study B, Paper IV)...................................... 50

Ethical considerations ........................................................................51

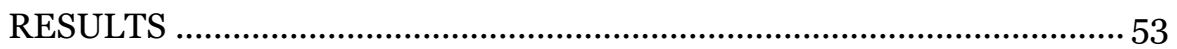


The Determinants of Implementation Behaviour Questionnaire (DIBQ) (Papers I and II) ................................................................. 53

Questionnaire development (Study A) ...................................................53

Longitudinally evaluation with DIBQ (Paper II)...................................56

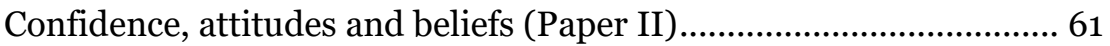

Adherence to clinical practice guidelines (Papers III and IV) ............. 62

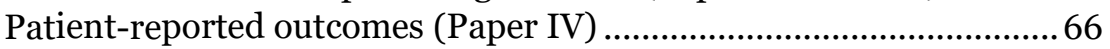

The effectiveness of implementation on patient-reported outcomes ....... 68

Guideline adherence effect on patient-reported outcomes ...................... 70

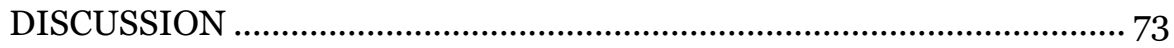

Main findings....................................................................................... 73

Evaluation of guideline implementation .............................................. 73

The implementation intervention........................................................... 74

Behaviour change is time dependent .................................................... 76

Desired behaviour can already exist ........................................................ 77

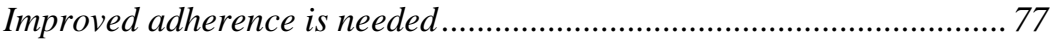

The multifaceted implementation strategy …………………………........ 77

Evaluation of the clinical intervention .................................................. 79

Patient-reported outcomes ................................................................... 79

The content of the BetterBack $\odot \mathrm{MoC}$..................................................... 80

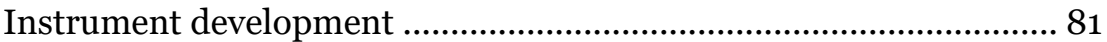

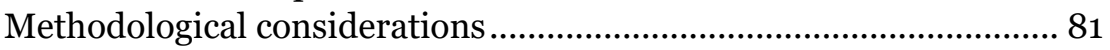

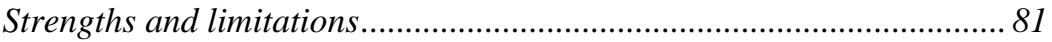

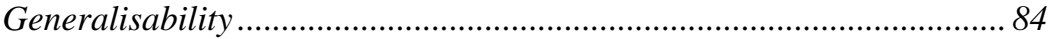

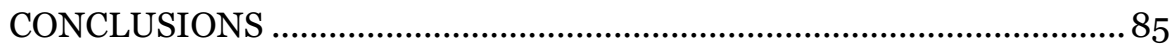

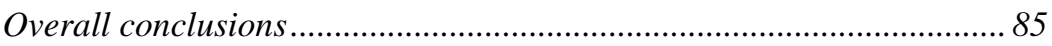

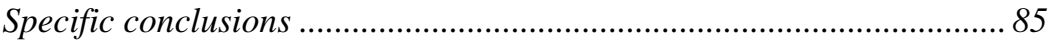

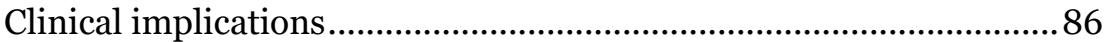

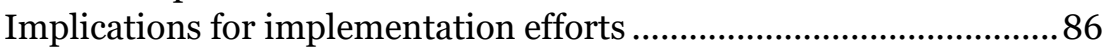

Future research …........................................................................... 87

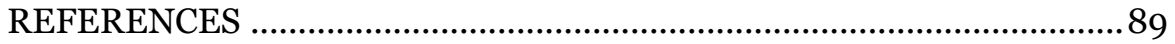




\section{ABSTRACT}

Background: Low back pain (LBP) occurs in all ages, and first-line treatment by physiotherapists (PTs) is common. However, national evidence-based LBP clinical guidelines are still lacking in Sweden. To facilitate guideline uptake, we developed and with a multifaceted strategy implemented a best practice physiotherapy healthcare model (BetterBack(:) MoC) with the aim of supporting management of LBP in primary care.

The overall aim of this thesis was to evaluate a multifaceted implementation strategy and a best practice physiotherapy healthcare model for LBP.

Methods: This thesis is based on one methodological study and three experimental trials with PTs and patients with LBP. In Paper I a mixed method design was used to translate, tailor, validate and feasibility-test the Determinants of Implementation Behaviour Questionnaire (DIBQ). This tailored DIBQ Questionnaire (DIBQ- $t$ ) was used to evaluate potential barriers/facilitators during the implementation process together with evaluation of PTs' confidence, attitudes and beliefs in managing LBP. In a stepped cluster randomised controlled trial, PTs and their patients in three clusters were allocated to intervention group (after implementation of BetterBack $\odot$ MoC) or control group (routine physiotherapy care). The proportions of guideline-adherent care were compared between groups (Paper III). This evaluation was based on PTs' adherence to eight clinical practice recommendations and three clinical practice quality indices (CPQI). The overall CPQI containing the five most prioritised recommendations are: no referral to specialist care; no referral to medical imaging for benign LBP; use of educational interventions; use of exercise interventions; and no use of non-evidence-based physiotherapy. Finally, in Paper IV patient-reported outcome measures (PROMs) were compared between intervention (after the implementation of BetterBack $\odot$ - MoC) and routine care. In Paper IV an additional secondary analysis was performed, comparing PROMs based on whether or not PTs had delivered care that met all five criteria of the overall CPQI or not.

Results: A tailored, feasible and valid questionnaire was developed, DIBQ- $t$ to be used for evaluation of the implementation of LBP primary 
care programmes. After implementation workshop, PTs' $(\mathrm{n}=116)$ confidence increased, and PTs' attitudes and beliefs shifted towards a more biopsychosocial orientation. PTs had high expectations of the BetterBack:MoC, which decreased after using the MoC. When evaluating received treatment for 500 patients with LBP, a more frequent delivery of guidelineadherent care was seen after implementation $(\mathrm{n}=278)$ compared to routine care $(\mathrm{n}=222)$. The overall $\mathrm{CPQI}$ containing the five prioritised recommendations was fulfilled in $59 \%$ of all patients in intervention versus $26 \%$ in routine care group. Analysis of adherence to specific recommendations showed a significantly improved use of stratification of number of PT visits and patient educational interventions, frequent use of exercise was maintained, and use of non-evidence-based treatment and medical imaging decreased after implementation. The primary outcome, i.e. referral to specialist consultation, was low in both groups, with no between-group differences. After implementation of the BetterBack $:$ MoC, no between-group differences in PROMs were seen except for greater satisfaction with LBP care, greater improvement in illness perception and better health-related quality of life, compared with routine care. However, when PTs' care adhered to all five CPQI criteria, a greater improvement of most patient-reported outcomes was seen compared to patient care that did not adhere to all five CPQI criteria.

Conclusions: The implementation of a best practice physiotherapy healthcare model (BetterBack $\odot$ MoC) for LBP improved both clinician and patient outcomes. PTs increased use of guideline-adherent care, confidence and biopsychosocial orientation in managing LBP. Patients reported improved satisfaction, illness perception and health-related quality of life. The implementation did not decrease referral to specialist consultation from an initial low level; nor did it improve patients' disability and pain more than routine care. However, when guideline-adherent care was fulfilled, most PROMs improved. It is therefore important to highlight the importance of guideline-based primary care for improving patientreported LBP outcomes. 


\section{SVENSK SAMMANFATTNING}

Bakgrund: Ländryggssmärta (LS) förekommer i alla åldrar och behandling av fysioterapeut som första instans är vanligt. För närvarande saknas evidensbaserade nationella kliniska riktlinjer i Sverige. För att främja riktlinjeanvändning utvecklades och implementerades ett vårdprogram (BättreRygg@) för att stödja behandling av LS i primärvård.

Övergripande syfte med denna avhandling var att utvärdera en mulifacetterad implementeringsstrategi och ett fysioterapeutiskt vårdprogram för ländryggsmärta.

Metoder: Avhandlingen är baserad på en metodologisk studie och tre experimentella studier med fysioterapeuter och patienter med LS. I första delarbetet användes en "mixed method design" för att översätta, anpassa samt testa validitet och användarvänlighet av frågeformuläret Determinants of Implementation Behaviour Questionnaire (DIBQ). Denna anpassade version av DIBQ (DIBQ-t) användes i delarbete II för att utvärdera potentiella hinder/facilatorer under implementeringsprocessen tillsammans med utvärdering av fysioterapeuters självförtroende, attityder och uppfattningar vid bedömning och behandling av LS. I en stegvis klusterrandomiserad kontrollerad studie, delades fysioterapeuter och deras patienter från tre kluster in i en interventionsgrupp (efter implementering av vårdprogrammet BättreRygg $($ ) ) eller en kontrollgrupp (sedvanlig fysioterapeutisk vård). Andelen riktlinjebaserad vård jämfördes mellan grupperna (delarbete III). Denna utvärdering baserades på fysioterapeuters följsamhet till åtta riktlinje rekommendationer och tre kvalitets index. Det övergripande kvalitetsindexet innehöll de fem mest prioriterade rekommendationerna; ingen remittering till specialistvård; ingen remittering till medicinsk röntgen vid godartad LS; användning av undervisningsinterventioner; användning av träningsinterventioner; och ingen användning av icke evidensbaserad fysioterapi. Slutligen, i delarbete IV jämfördes patientrapporterade utfallsmått mellan intervention (patienter behandlade efter implementering av vårdprogrammet BättreRygg(:) och patienter som erhållit sedvanlig vård. En tilläggsanalys gjordes också i delarbete IV som jämförde patientrapporterade utfallsmått baserat på om fysioterapeuternas vård uppfyllde alla fem kriterierna för det övergripande kvalitetsindexet eller inte. 
Resultat: Ett anpassat, användarvänligt och valitt frågeformulär utvecklades, DIBQ-t, för att användas vid utvärdering av implementering av vårdprogram för LS i primärvård. Efter implementeringsworkshopen ökade fysioterapeuternas $(n=116)$ självförtroende samt en förändring av attityder och uppfattningar mot en mer biopsykosocial orientering kunde visas. Fysioterapeuterna hade höga förväntningar på vårdprogrammet som minskade efter praktiskt användande. Vid utvärdering av behandling för 500 patienter med LS framkom en mer frekvent användning av riktlinjebaserad vård efter implementering $(\mathrm{n}=278)$ jämfört med kontrollgruppen som erhållit sedvanlig vård $(n=222)$. Det övergripande kvalitetsindexet med de fem prioriterade rekommendationerna uppfylldes hos $59 \%$ av alla patienter i interventionsgruppen jämfört med $26 \%$ i gruppen som fătt sedvanlig vård. Analys av följsamheten till specifika rekommendationer visade en signifikant förbättrad användning av stratifiering av antal besök och patientundervisningsinterventioner, hög användning av träning bibehölls, och en minskning av icke evidensbaserad fysioterapi och medicinsk röntgen efter implementering. Det primära utfallsmåttet, remittering till specialistvård var låg i båda grupperna utan mellangruppsskillnad. Efter implementering av vårdprogrammet BättreRygg(:) fanns ingen mellangruppsskillnad avseende patientrapporterade utfallsmått förutom högre tillfredställelse med sin ryggvård, större förbättring av uppfattningar av ryggbesvär och förbättrad hälsorelaterad livskvalitet, jämfört med sedvanlig vård. När fysioterapeuternas vård innehöll alla fem rekommendationerna i det övergripande kvalitetsindexet, förbättrades de flesta patientrapporterade måtten mer jämfört med vård som inte innehöll de fem rekommendationerna.

Konklusioner: Implementering av ett fysioterapeutiskt vårdprogram (BättreRygg(-) ) för LS förbättrade både klinik- och patientrapporterade utfallsmått. Fysioterapeuterna ökade användningen av riktlinjebaserad vård, förbättrade självförtroende och biopsykosocial orientering vid bedömning och behandling av LS. Patienterna rapporterade förbättrad tillfredställelse, uppfattningar av ryggbesvär, och hälsorelaterad livskvalitet. Implementeringen minskade inte remittering till specialistvård från den initialt låga nivån och förbättrade inte patienternas funktion och smärta mer än sedvanlig vård. Däremot, förbättrades de flesta patientutfallsmått när vården var riktlinjebaserad. Det är därför viktigt att poängtera vikten av riktlinjebaserad primärvård för att förbättra patientrapporterade utfallsmått vid LS. 


\section{LIST OF PAPERS}

I. Ris I, Schröder K, Kongsted A, Abbott A, Nilsen P, Hartvigsen J, Öberg B. Adapting the Determinants of Implementation Behaviour Questionnaire to evaluate implementation of a structured low back pain programme using mixed-methods. Health Sci Rep. 2021;4:e266. http://doi.org/10.1002/hsr2.266

II. Schröder K, Öberg B, Enthoven P, Kongsted A, Abbott A. Confidence, attitudes, beliefs and determinants of implementation behaviours among physiotherapists towards clinical management of low back pain before and after implementation of the BetterBack model of care. BMC Health Serv. Res. 2020;20:443.

https://rdcu.be/b4hBn

III. Schröder K, Öberg B, Enthoven P, Hedevik H, Abbott A. Improved adherence to clinical guidelines for low back pain after implementation of the BetterBack model of care: A stepped cluster randomized controlled trial within a hybrid type 2 trial. Manuscript

IV. Schröder K, Öberg B, Enthoven P, Hedevik H, Fors M, Abbott A. Effectiveness and Quality of Implementing a Best Practice Model of Care for Low Back Pain (BetterBack) Compared with Routine Care in Physiotherapy: A Hybrid Type 2 Trial. J. Clin Med. 2021;10(6):1230. https://doi.org/10.3390/jcm10061230 


\section{DESCRIPTION OF CONTRIBUTION}

\section{Paper I}

Study Design:

Karin Schröder, Inge Ris, Alice Kongsted, Allan

Abbott, Per Nilsen, Jan Hartvigsen, Birgitta Öberg

Data Collection: Karin Schröder, Inge Ris, Birgitta Öberg, Alice Kongsted, Allan Abbott

Data Analysis: $\quad$ Karin Schröder, Inge Ris, Birgitta Öberg, Allan Abbott, Henrik Hedevik

Manuscript Writing: Inge Ris, Karin Schröder, Allan Abbott, Per Nilsen, Jan Hartvigsen Birgitta Öberg

Manuscript Revision: Inge Ris, Karin Schröder, Alice Kongsted, Allan Abbott, Per Nilsen, Jan Hartvigsen, Birgitta Öberg

Corresponding author: Inge Ris

\section{Paper II}

Study Design:

Karin Schröder, Birgitta Öberg, Paul Enthoven, Alice Kongsted, Allan Abbott

Data Collection: Karin Schröder

Data Analysis:

Karin Schröder, Birgitta Öberg, Paul Enthoven, Allan Abbott, Henrik Hedevik

Manuscript Writing: Karin Schröder

Manuscript Revision: Karin Schröder, Birgitta Öberg, Paul Enthoven, Alice Kongsted, Allan Abbott

Corresponding author: Karin Schröder

\section{Paper III}

Study Design:

Data Collection:

Data Analysis:

Manuscript Writing

Manuscript Revision:

Corresponding author:
Karin Schröder, Birgitta Öberg, Paul Enthoven, Allan Abbott

\section{Karin Schröder}

Karin Schröder, Birgitta Öberg, Paul Enthoven, Henrik Hedevik, Allan Abbott

\section{Karin Schröder}

Karin Schröder, Birgitta Öberg, Paul Enthoven, Henrik Hedevik, Allan Abbott

\section{Karin Schröder}




\section{Paper IV}

Study Design:

Karin Schröder, Birgitta Öberg, Paul Enthoven, Allan Abbott

Data Collection: $\quad$ Karin Schröder, Maria Fors, Johan Nystrand

Data Analysis:

Karin Schröder, Birgitta Öberg, Paul Enthoven, Henrik Hedevik, Maria Fors, Allan Abbott

Manuscript Writing: Karin Schröder

Manuscript Revision: Karin Schröder, Birgitta Öberg, Paul Enthoven, Henrik Hedevik, Maria Fors, Allan Abbott

Corresponding author: Karin Schröder 
Implementing BetterBack - a Best Practice Physiotherapy Healthcare Model for Low Back Pain 


\section{ABBREVIATIONS}

$\begin{array}{ll}\text { AUC } & \text { Area under the curve } \\ \text { BCW } & \text { Behaviour change wheel } \\ \text { BIPQ } & \text { Brief Illness Perception Questionnaire } \\ \text { BM } & \text { Biomedical } \\ \text { BPS } & \text { Biopsychosocial } \\ \text { CFA } & \text { Confirmatory factor analysis } \\ \text { CFI } & \text { Comparative fit index } \\ \text { CI } & \text { Confidence interval } \\ \text { COM-B } & \text { Capability opportunity motivation-behaviour system } \\ \text { COSMIN } & \text { COnsensus-based Standards for the selection of health } \\ & \text { Measurements INstruments } \\ \text { CPG } & \text { Clinical practice guideline } \\ \text { CPQI } & \text { Clinical Practice Quality Index } \\ \text { CRPE } & \text { Clinical Reasoning and Process Evaluation tool } \\ \text { CSM } & \text { Common-sense model of self-regulation } \\ \text { CT } & \text { Computerised tomography } \\ \text { CVI } & \text { Content validity index } \\ \text { DIBQ } & \text { Determinants of Implementation Behaviour Questionnaire } \\ \text { DIBQ-t } & \text { Determinants of Implementation Behaviour Questionnaire } \\ & \text { - tailored } \\ \text { EBP } & \text { Evidence-based practice } \\ \text { EQ-5D } & \text { EuroQol-5 Dimensions scale } \\ \text { ICC } & \text { Intracluster correlation coefficient } \\ & \text { ICD-10 International statistical classification of diseases } \\ \text { ICF } & \text { and related health problems } \\ & \text { International classification of functioning, disability, and } \\ \text { LBP } & \text { health } \\ \text { MCID } & \text { Low back pain } \\ \text { MoC } & \text { Minimal clinically important difference } \\ \text { MRI } & \text { Model of care } \\ \text { NICE } & \text { Magnetic resonance imaging } \\ \text { NRS-LBP } & \text { National Institute for Health and Care Excellence } \\ & \text { Numeric Rating Scale for low back pain } \\ & \end{array}$


ODI Oswestry Disability Index

OCP Optimal cut-off point

OR Odds ratio

PABS Pain Attitude and Beliefs Scale

PCS Practitioner Confidence Scale

PEI Patient Enablement Instrument

PGIC Patient Global Impression of Change

PHRR Public health regional registry

PROM Patient-reported outcome measure

PS Patient Satisfaction

PT Physiotherapist

RCT Randomised controlled trial

RMANOVA Repeated measures analysis of variance

RMSEA Root mean square error of approximation

ROC Receiver operating curve

SBT STarT Back Screening Tool

SD Standard deviation

SRMR Standardized root mean squared residual

TDF Theoretical Domains Framework

TIDieR Template for intervention description and replication checklist

TLI Tucker-Lewis index

ÖMPSQ Örebro Musculoskeletal Pain Screening Questionnaire 


\section{ACKNOWLEDGEMENTS}

First, I would like to thank all of you who have contributed to this work in different ways, and who have supported me throughout my PhD studies. I would especially like to thank:

Allan Abbott, main supervisor, for your patience and your valued support. Thank you for unselfishly sharing your great knowledge in the low back pain field, and your statistical and writing skills.

Birgitta Öberg, supervisor, for knowing how to challenge me, and your extraordinary ability to take fragments, and to re-interpret them as a whole picture.

Paul Enthoven, supervisor, for valuable feedback, and your ability to see details in manuscripts, Power Point presentations and other texts.

Alice Kongsted, supervisor, for having an eye for structure and the ability to explain complicated things in an easily understood way. Thank you for inviting me to Denmark to participate in the GLA:D ${ }^{\circledR}$ Back course and to visit your family.

Henrik Hedevik, statistician, and co-author, for statistical support and for being so precise and rigorous in your work. A special thanks for the idea of using a clinical practice quality index in Papers III and IV.

Inge Ris Hansen, co-author, for sharing knowledge and experiences from your research group, always with enthusiasm.

Danish research team, Alice Kongsted, Inge Ris, Jan Hartvigsen and Per Kjaer for your deep knowledge in low back pain research that you always share with us. Thank you for collaboration in developing DIBQ-t and giving us lots of fruitful input along the way. I hope for a continued collaboration and more face-to-face visits in the future.

Åse Engholm, Angelica Ivarsson, Fariba Gharachorloo, Nina Dahlholm and Lennart Sandelius, Clinical champions in initial MoC support team, 
for enthusiasm, valuable input from the "real world" and for the acting as local ambassadors for the BetterBack $\odot$ MoC during the implementation.

Physiotherapists in the "Development and sustainability group" for valuable inputs in developing and sustaining the BetterBack@) MoC.

All participating physiotherapists, for taking time to include patients and answer questionnaires. Without your efforts this research would not have been possible.

Maria Fors and Johan Nystrand for helping collect patient questionnaires. Thank you, Maria, for fruitful discussions along the whole PhD journey and valuable inputs on Paper IV.

Rehabilitation managers in primary care, Östergötland, who supported us along the way and especially for making the implementation workshop mandatory for all physiotherapists.

My fellow PhD students, colleagues and administrative staff at the unit of Physiotherapy, Linköping University, for interesting and valuable discussions, and positive support.

Friends who have patiently listened to me when needed, and for all the fun during these years, reminding me that I am not a PhD student, I just work as one.

Jan Schröder, my husband, for love, being a great listener, always believing in me and for an active happy life outside the sometime absorbing $\mathrm{PhD}$ work.

My family Emil Schröder, Eric Schröder and Carl Schröder with your partners Emma Sandström, Sofia Wikberg and Tilda Nestun. When being with you I feel refilled with love and energy, and I forget unfinished manuscripts, statistical problems and unsolved research questions.

Frost, our dog who has been the best working mate during these years, especially during times of social distancing. Always sleeping beside my desk, and forcing me to take long lunch breaks in the forest.

Parents and relatives for being there and supporting me along the way. 


\section{INTRODUCTION}

In Sweden and many other countries, patients with low back pain (LBP) are one of the most common patient groups in primary care, and physiotherapists (PTs) are often their first contact with healthcare. Following the evidence-based practice movement in the 1990s, researchbased evidence became increasingly important in physiotherapy. Treatment interventions for LBP changed from mainly passive strategies to predominantly active interventions (1). Implementation science as a research field also grew rapidly in the light of the evidence-based practice movement. To bridge the gap between evidence and practice, numerous guidelines for managing LBP started to develop. However, use of guidelines in physiotherapy has been a challenge, with less than half of the Swedish PTs in Västra Götaland County's primary care setting reporting guideline use in 2014 (2). At the same time, studies showed use of non-evidencebased interventions among Swedish primary care PTs (3) and insufficient primary care physiotherapy before spinal surgery (4).

In 2016, healthcare managers in the region of Östergötland issued a request to improve LBP physiotherapy, as they experienced that LBP care was fragmented with inequitable quality. This was the start of the development of the BetterBack $:$ model of care, and the start of my PhD journey. After more than 25 years as a physiotherapist and 10 years as a manager for a private physiotherapy clinic, I needed a challenge. I agreed to be a part of the BetterBack $\odot$ research team 2016, and began my $\mathrm{PhD}$ studies in 2017. In 2016 a team of researchers and selected 'clinical champions' from involved rehabilitation centres in Östergötland adapted existing international LBP guidelines to local conditions and incorporated them into a model of care (MoC). This $\mathrm{MoC}$ was named BetterBack -$)^{-}$The next step was to implement the BetterBack - MoC during a mandatory twoday workshop in Östergötland Region's publicly financed primary care physiotherapy.

After the initial development phase, our research group collaborated with a Danish research group who developed a similar care package for a Danish primary care context (5). There were few translated instruments suitable for evaluating implementation behaviours among clinicians. Therefore, in Study I our research groups collaborated with the Danish research group in translating and tailoring, as well as feasibility and validity-testing of an existing instrument, the Determinants of Implementation Behaviours Questionnaire (DIBQ). This adapted instrument DIBQ-t (DIBQ tailored), was then used in Study II. This thesis 
is the first in Sweden to evaluate the effectiveness of implementing a best practice physiotherapy healthcare model for LBP with evaluation of physiotherapists' confidence, attitudes and beliefs and adherence to guideline recommendations together with patient outcomes, with the aim of improving LBP care in primary care. 


\section{BACKGROUND}

\section{Low back pain}

Low back pain is a common symptom in populations worldwide, and it occurs in all ages $(6,7)$. LBP is commonly described by its location and symptoms of pain and discomfort below the costal margins and above the inferior gluteal folds, or back pain with or without radiculopathy (8). Back pain has also been described by its intensity, frequency, influence on activity and duration (9). However, the accepted duration classification acute 0-3 weeks, subacute 4-12 weeks, and chronic pain $>12$ weeks (10) has recently been challenged, following findings that back pain fluctuates over time with symptoms on and off for years $(11,12)$. Up to $90 \%$ of all LBP cases can be categorised as non-specific, where the underlying pathology or disease cannot be identified through diagnostic assessment (13). In primary care approximately $<1 \%-4 \%$ of LBP cases are non-benign and show signs of underlying malignancy, cauda equina syndrome, fracture or infection $(14,15)$. Furthermore 5-15 \% of all cases have neuropathic pain presentation clinically as lumbar radiculopathy or lumbar spinal stenosis $(16,17)$. Medical imaging is still common and increasingly utilised as a diagnostic assessment method for LBP, but is problematic since spinal degeneration is present in high proportions of asymptomatic individuals (18). Studies have shown that $37 \%$ of 20-year-old asymptomatic individuals and $96 \%$ of 80-year-olds have disc degeneration findings (19). Multiple factors have been described to contribute to LBP, and LBP has been described as more typically a result of benign biological and psychosocial dysfunctions with social contextual factors influencing the complex pain experience (6). In conclusion this complexity of LBP needs to be addressed in diagnosing and treating LBP with a broad biopsychosocial approach.

\section{Prevalence and impact on health and welfare}

The lifetime prevalence of LBP is high, ranging from $51 \%$ to $84 \%$ (20) with a reported point prevalence of $12 \%(7)$. LBP occurs in all ages and is more common among females and ages 40-80 years (7). Globally, years lived with disability caused by LBP has increased by $54 \%$ between 1990 and 2015, and is now the number one cause of disability globally (6). Most people with a new episode of LBP recover quickly, however recurrence is common and for a smaller proportion of people, pain becomes persistent (6). The prevalence of persistent LBP has been reported to range from $3.9 \%$ 
to $25.4 \%$ in individuals aged 18 and above depending on age group and socioeconomic status (21). The high costs of LBP for society can be explained by the costs of both loss of working ability and costs for healthcare (22). Patients with LBP in Sweden consume nearly twice as much healthcare than the general population (23). In Sweden and many other countries most patients with LBP are treated in primary care, and for approximate $70 \%$ of patients with LBP a PT is the initial care contact (24). The consequences of LBP are considerable for the individuals, healthcare system and society, which highlights the need for awareness and improved knowledge, in order to reduce the burden of LBP.

\section{Pathogenesis, risk, and prognosis}

LBP is nowadays described as multi-factorial, with involvement of many different factors and mechanisms contributing to its cause and its recurrence (25). In recent decades the biopsychosocial model has been the dominant model for understanding the aetiology and prognosis of back pain (26). However, a critical review showed that PTs' main focus when managing LBP was on biological aspects, at the expense of social and broader aspects such as culture and interpersonal dynamics (27). Although clinical tests and medical imaging are unable to identify the accurate tissue source of most LBP, several innervated structures such as intervertebral disc, facet joint and vertebral endplates have been shown to reproduce pain when stimulated (6). Other biological causes in persistent LBP are central nervous system sensitisation and abnormal central processing of pain (28). Psychological and social factors have been shown to modulate pain in the pain neuromatrix (29). Recently, epigenetics has also been suggested as a potentially important biological mechanism in back pain (30).

Hartvigsen et al. (6) have presented a model of multifactorial contributions to LBP and disability. In this model, key contributions are shown but with no attempt to show the complex interactions between the different contributors (Figure 1). However, the biopsychosocial model shows how biological, social and psychological factors influence pain but do not inform the prognosis. Therefore, different risk stratification assessment screening tools have been developed. However, a review by Karran et al. (31) showed that LBP screening instruments in primary care perform poorly at assigning high risk scores to individuals who develop chronic pain. The two most common and recommended screening tools (32) are the STarT Back Screening Tool (SBT) (33) and the Örebro Musculoskeletal Pain Screening Questionnaire (ÖMPSQ) (34). ÖMPSQ assists in the early identification of yellow flags (psychosocial factors) and patients at risk of developing work disability (34). SBT is designed for primary care and predicts long-term disabling pain. 


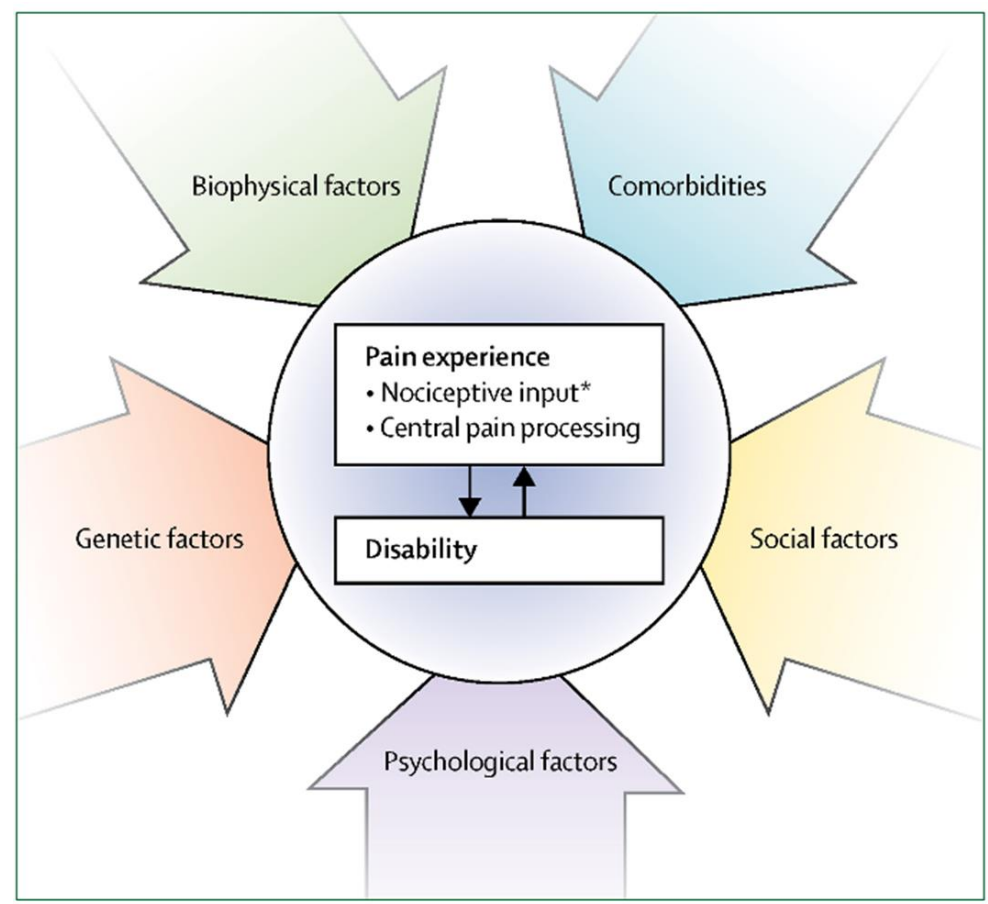

Figure 1. Contributors to low back pain and disability (with permission from Elsevier).* Nociceptive input includes non-identifiable sources in non-specific low back pain, neurological sources and specific pathology.

\section{Evidence-based practice (EBP)}

\section{Clinical practice guidelines (CPG)}

Over the last decades, a myriad of treatment options and a growing body of clinical trials and scientific publications for LBP have emerged. To be able to offer evidence-based healthcare, it is important that healthcare practitioners (HCP) involved in LBP care have easy access to up-to-date, evidence-based information to assist in treatment decision-making (35). Clinical practice guidelines (CPGs) also called 'clinical guidelines' can improve healthcare by promoting best practice, reducing the use of lowvalue interventions and unwarranted variations of healthcare (35). According to the Institute of Medicine, CPGs are defined as:

"Statements that include recommendations intended to optimize patient care that are informed by a systematic review of evidence and an assessment of the benefits and harms of alternative care options" (36) 
The term guideline is more general and broader, developed and used by service organisations, policymakers, and authorities. CPGs are in contrast typically intended for use by healthcare providers to support their clinical decisions (37). However, the terms CPGs and guidelines are often used interchangeably, as they are in this thesis. In the field of LBP, CPGs have been rapidly developed.

O'Connell et al. (35) have compared major international LBP guidelines. The consistent recommendations across the guidelines were diagnostic triage with red flag screening, exercise therapy, patient information, and education (35). The information and education should be directed towards an "expected" good prognosis to help moderate patients' expectations and to reduce risk of fear/catastrophising. Furthermore, core recommendations are advice to stay active and return to normal activities as soon as possible. Manual therapies and psychological interventions were recommended in the various guidelines, but in the National Institute for Health and Care Excellence (NICE) guidelines are to be used only as a part of a treatment package and not as an essential component for all patients (38). All guidelines recommended some form of exercise therapy; the NICE guidelines placed exercise therapy at the centre of LBP treatment to a greater extent than the others. Across all guidelines, multi-modal care options including self-management approaches in the management of pain-related symptoms were recommended. All guidelines recommended against routine use of interventions such as imaging, opioids, spinal surgery, spinal traction, and bedrest for patients with benign LBP. Recommendations were not consistent in the guidelines regarding use of clinical pathways with a stepped care (all patients are offered the same basic care and treatment efforts are only increased if patients do not improve sufficiently) or use of risk stratification approach (35). In the risk stratification approach, individuals are matched with appropriate target treatments according to their risk profile in the three risk groups low, medium and high, according to SBT (39). Individuals in low-risk groups with a good prognosis are advised to stay active, informed of their overall good prognosis, educated, and informed to use self-management strategies. The medium-risk group individuals with a possible poor prognosis are offered an additional evidence-based physiotherapy such as exercise and manual therapy. High-risk group individuals with a probable poor prognosis with significant impact and distress are offered the same as low-risk individuals plus a more complex treatment including a deeper biopsychosocial assessment with both physical and psychological interventions. This complex treatment will require a higher number of healthcare visits. An SBT stratification approach with both screening and matching treatment has been shown to improve LBP care and save costs (40), and in a recent systematic review and meta-analysis this was evident 
for medium- and high-risk patient groups but not low-risk groups (41). Despite lack of evidence for long-term benefits, the SBT approach is suggested to be part of a well-designed model of care for LBP $(41,42)$. In 2021 a new systematic review of European LBP and neck pain guidelines by Corp et al. (43) recommended entirely non-pharmacological interventions for benign back pain. This review showed similar results as a previous review by O'Connell et al. (35), with additional recommendations on return-to-work programmes (moderate evidence), group exercise program including back schools (moderate evidence) and for specific subgroups both psychological therapies (moderate evidence), and multidisciplinary treatments (strong evidence). Furthermore, manual therapy was now recommended in combination with other treatments (moderate evidence). This review also included a recommendation against orthoses and electrotherapies, and for acupuncture the recommendation was inconsistent (43). In both reviews, the dosage, duration, and other details around delivery of the recommended treatments, and consensus recommendations about clinical pathways, were not described $(35,43)$. In 2020, Castellini et al. (44) reviewed the quality of different LBP guidelines and found that the NICE guidelines (38) had the highest quality (96\%) in the area of educational/behavioural, physiotherapy and pharmaceutical interventions.

\section{Barriers in CPG implementation}

Despite guidelines with clear and consistent recommendations, there is still an evidence-to-practice gap for LBP care internationally with poor uptake of many of these assessment/diagnostic-related and treatment recommendations (6). This gap can be due to the challenge of implementing back pain guidelines in clinical practice, and many barriers to clinical guideline implementation have been identified (45). Less focus has been on facilitators for CPG implementation. However, consistent leadership, committed team members, administrative support and education regarding the guidelines have been shown to be facilitators (46). Barriers in implementation of LBP guidelines in physiotherapy have been described, such as challenges in integrating guidelines in clinical practice, allocation of time, and expectations from patients and managers $(35,47)$. Some clinicians consider guidelines a threat to their professional autonomy (48). Many clinicians continue to manage patients with LBP with a biomedical orientation, and this appears to negatively impact upon adherence to treatment guidelines which nowadays have a biopsychosocial approach (48). This highlights the importance of addressing potential barriers and facilitators when planning and evaluating guideline implementation. 


\section{Low back pain CPG implementation in primary care physiotherapy}

Implementing LBP guidelines in clinical practice is challenging because guidelines are just one component in a more multi-component and complex process of translating research into clinical practice (49). Furthermore, implementation success depends to a high degree on the contextual factors, which limits the generalisability between different health settings, countries and healthcare systems (50). Like other healthcare disciplines, it has been evident that there are challenges in guideline implementation in the physiotherapy context, with difficulties improving adherence to these guidelines (51). Adherence has been described as the extent a person's behaviour corresponds to recommendations (52). It is suggested that training and support tools would facilitate the use of this management approach (53). Use of a biomedical management orientation has been found to be associated with poor adherence to recent LBP guidelines (54) as well as advices to delay a return to normal activities and work for patients with LBP (55). A systematic review on how PTs follow evidence-based guidelines based on surveys completed by PTs showed that a median percentage of $85 \%$ of all PTs provide or would provide LBP treatments that were recommended (56). Furthermore, the median percentage of PTs who provided or would provide LBP treatments that were not recommended was $38 \%$, however when data was based on what the patients received from treatment records and billing codes, $79 \%$ of patients had received non-recommended treatments (56).

In Sweden Bernhardsson et al. (2) showed that $96 \%$ of a cohort of Swedish primary care PTs considered guidelines important, but fewer than half reported using guidelines frequently. After a tailored, multicomponent guideline implementation, Bernhardsson et al. (57) showed an increased awareness and knowledge of where to find the local LBP, neck pain and shoulder pain guidelines, but no significant effect on guideline use. Fritz et al. (58) have recently evaluated a multifaceted implementation of a behavioural approach for persistent musculoskeletal pain including LBP in primary care. This small quasi-experimental pre-/post trial with a total of 24 PTs and 159 patients showed a significantly changed clinical behaviour among PTs immediately after the implementation period, but this effect did not remain to the three-months follow-up (58). Patients treated by these trained PTs in the behavioural approach did not achieve further improvements compared to a control group (59). In Sweden there is a lack of national clinical guidelines for LBP, and Swedish PTs still consider the use of interventions despite lack of evidence or treatment effects (3). Furthermore, evidence-based physiotherapy interventions have been shown to be underused. In a Swedish study from the healthcare region 
of Östergötland, only $58 \%$ of patients on surgical waiting lists saw a PT within one year prior to a spine surgeon consultation, despite guideline recommendations to exhaust non-surgical interventions before surgery (4). A qualitative study showed that these LBP patients after a pre-surgery physiotherapy intervention with cognitive behavioural approach still describe their back-related symptoms in line with a biomedical explanatory model (60).

\section{Implementation Science}

The term implementation is used to describe the process of integrating evidence-based interventions within a setting or of bridging the researchto-practice gaps (61). In North America the term knowledge translation is more commonly used, while in other parts of the world the term implementation is more frequently used, as it is in this thesis. A new intervention/innovation can be spread a long a continuum (Figure 2), where diffusion can be described as a passive and unplanned informal spread of a new practice. While dissemination is an active spread using formal and planned strategies implementation at the end of the continuum is the process where new practice integrates within the actual setting (61).

\section{Diffusion Dissemination Implementation}

"let it happen" "help it happen" "make it happen"

Figure 2. The diffusion to implementation continuum, based on Greenhalgh et al. (62)

In recent research, a greater focus has been on what happens after implementation with addressing sustainability along the continuum (63). It is important to distinguish between implementation science and intervention research. Implementation research focusses on strategies used to implement evidence-based practice, while intervention research focusses on intervention effectiveness (64). Implementation Science has been defined as:

"applied research that aims to develop the critical evidence
base that informs the effective, sustained and embedded
adoption of interventions by health systems and communities." (65) 


\section{Behaviour change}

The problems of getting clinicians to act on evidence-based guidelines are widely recognised. In implementation-related, evidence-based practice, the target population must undergo a behaviour change if it is to adopt to a new practice. An increased focus on behaviour change in guideline implementation has been suggested; this involves specifying clinicians/patients target behaviour and with a behavioural analysis to assist in developing effective interventions (66). To help with understanding behaviour and behaviour change, different models and frameworks can be used. One such model is the behaviour model (capability, opportunity and motivation) termed COM-B model (67). In this model capability, opportunity and motivation interact over time and generate a behaviour. This model is also used when planning, implementing and evaluating behavioural interventions, and it aims to identify the appropriate targets for the behaviour change interventions (67, 68).

Other behaviour change frameworks aiming at implementation research have been developed. The Theoretical Domains Framework (TDF), which is a synthesis of 33 theories of behaviour and behaviour change models into originally 12 TDF domains, is one of the most common (69). The TDF was developed to help implementation researchers to identify influence on HCPs' behaviour related to implementation of evidence-based recommendations $(69,70)$. The TDF can be used to identify attitudinal and behavioural barriers and facilitators of uptake of behaviour change in clinical interventions and to assist in the development of effective implementation strategies (71).

In the behaviour change wheel (BCW), both the COM-B and the TDF are integrated (72) (Figure 3). The COM-B model acts here as a hub identifying the source of the target behaviour for the intervention with linkage to TDF domains. Surrounding this is a layer of intervention functions to choose from depending on the result of the COM-B analysis. Then the outer layer consists of different policy categories that one can use to deliver these intervention functions $(67,73)$. The aim of the BCW is to allow researchers to use a systematic method for understanding behaviour and linking this understanding to techniques known to change behaviour. The BCW is commonly used in designing and evaluating behaviour change interventions $(72,74)$. The use of the BCW is suggested in a framework by Briggs et al. (75) intended to assist in planning, implementing and evaluating phases for implementation of musculoskeletal model of care. 

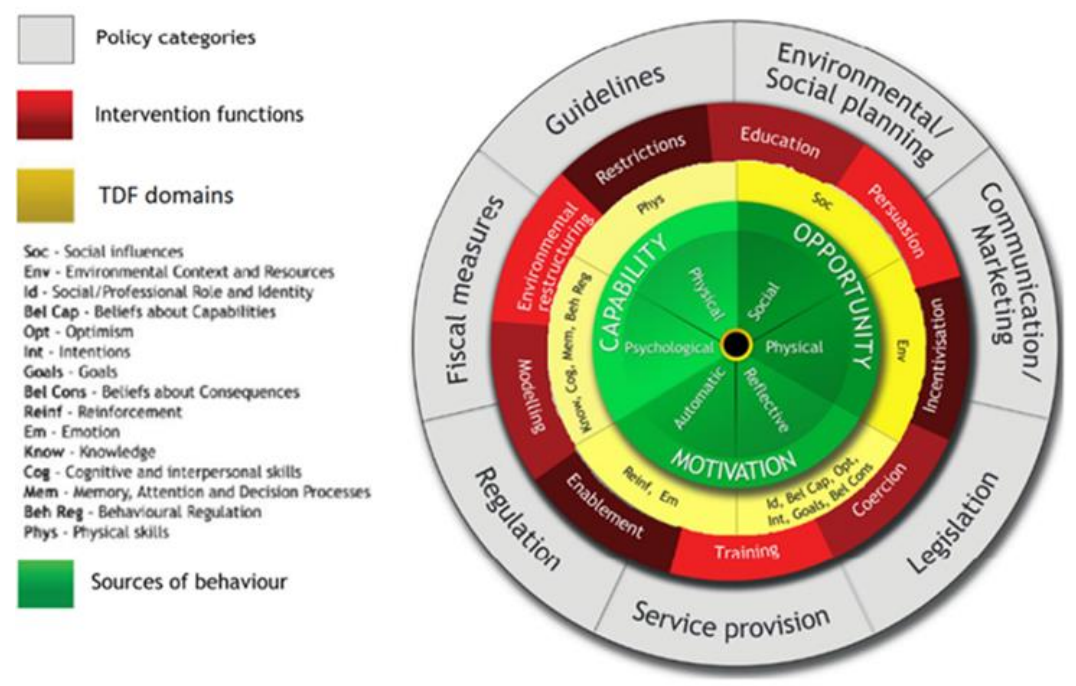

Figure 3. The behaviour change wheel (BCW) (76)

\section{Model of care}

A model of care (MoC) provides a framework for implementing evidence into practice, describing the principles of evidence-informed healthcare delivered to consumers in the actual setting for a specific disease. The right care should be delivered at the right time, in the right place, by the right team using the right resources (77). A MoC describes not only what to deliver, but also how to deliver it within a healthcare system and serves as a complement to clinical practice guidelines (77).

\section{Implementation theories and models}

Use of theories and models can support the analysis of potential success and of factors related to outcomes in implementation processes (78). Various theories and models for change point to a large number of factors that may affect successful implementation of evidence. There has been an increasing use of theories to assist in designing or evaluating interventions, and according to a scoping review in 2017, half of the published guideline implementation studies were based on theory (79). In a review by Tabak et al. (80) 91 different models were identified, showing that there is a vast amount to choose from. Prihodova et al. (81) reviewed over 60 models in health service research and found six key components used in the models. The first primary key component was the Message that should be need-driven, accessible and credible. Moreover it should use of different methods to deliver the message. The second key component was Process - timely, targeted and multifaceted activities to implement the transfer of knowledge using 'push-pull' dynamic exchanges of information 
with involved opinion leaders/champions involved. The third key component, the Stakeholders, represents involvement of people on either side of the exchange process here also with involvement and support from leaders and managers in the organisation. The two contextual key components were Inner Context (often representing the organisational environments) and the wider Social, Cultural and Economic Context. The final component is the importance of Evaluating Efficacy of the implementation activity. The authors used these six key components and created a new synthesized model to be used when planning implementation activities, however a need for further studies of this new model was suggested (81).

There are also different frameworks to assist in planning the implementation, often with checklists of important factors and aspects when implementing a model of care. BetterBack $\odot$ MoC has used the framework by Briggs et al. (75). This framework has been developed for implementation of musculoskeletal models of care to assist in both planning and implementation and evaluating phases with the components involved suggested by Prihodova et al. (81). This framework includes checklists for: (1) development of a clear model of care document (Message) acceptable to the local stakeholders; (2) judgement whether a model of care is ready for implementation; (3) guidance of the initial implementation process with different stakeholders involved, and adapted to the local context (Process, Stakeholders, Local context); and (4) how to consider performance measures that are likely to indicate to what degree the model of care is successful (Evaluating Efficacy) (75).

\section{Implementation strategy and evaluation}

Implementation strategies are the methods used to facilitate the uptake of EBP or research findings into routine practice (82). The effectiveness of different implementation strategies such as educational events, use of clinical champions, and passive or active implementation in LBP guideline implementation is unclear. However, it seems that increased duration and frequency of the implementation intervention leads to greater success (83).

Evaluation during and after implementation is important. To understand failure (or success) of transferring evidence into clinical practice, it is critical to distinguish implementation effectiveness from treatment effectiveness. This to understand if a failure (or success) was caused by an intervention that was ineffective in the actual setting (intervention failure), or was a good intervention deployed incorrectly (implementation failure) - or was it a combination of both (84). In effectiveness studies, evaluations focus on patient-level health and functioning outcomes while in implementation research evaluations focuses on the system-level with adoption, cost, fidelity, reach and 
sustainability as common outcomes (85). Adoption is described as uptake, utilisation, or the action to try the innovation or EBP, while fidelity is defined as the degree to which an intervention was implemented as described in the protocol (84). Three types of fidelity have been described by Proctor et al. (84): 1) adherence to the program protocol; 2) dose or amount of program delivery and 3) quality of delivery. Reach is described as the absolute number of individuals who are willing to participate (86), and sustainability as the extent to which the newly implemented treatment is maintained (84).

An implementation intervention aims to change the behaviour by targeting HCP, organisations, or systems, in contrast to a clinical intervention, which aims to affect patients. Distinguishing between these two is not always easy, and hybrid designs which address both the implementation intervention, and the clinical intervention are common (87). These hybrid designs will typically take one of three design types: I) primarily testing effects of a clinical intervention on relevant outcomes while observing and collecting information on implementation, II) dual testing of both the clinical and implementation interventions or III) primarily testing an implementation intervention while observing and collecting information on the clinical intervention's impact on outcomes (64). In this thesis a hybrid type II design has been used. In the protocol (76), outcomes are divided into implementation process, implementation, and patient outcomes. In this thesis outcomes are described and reported as implementation intervention outcomes targeting PTs and clinical intervention outcomes targeting patients.

Implementation outcomes can be measured by using administrative data and clinical indicators. Clinical indicators have a long tradition of measuring the quality of patient care delivered $(88,89)$. A clinical indicator measures the quality of care, and relies on adequate research evidence, clinical effectiveness, safety, and efficiency (89).

\section{Measurement properties in questionnaire development}

When using a questionnaire to measure something, it is important to examine its design and if it is suitable for use in the intended population. Therefore, it is important to test and evaluate the measurement properties with appropriate methodology (90). The most common measurement properties in medicine are reliability, validity and responsiveness. There is an international consensus among the COSMIN group (COnsensus-based Standards for the selection of health Measurements INstruments) on defining these properties (91). Reliability is defined as "the degree to which the measurement is free from measurement error" with the three measurement properties: internal consistency (often tested by a Cronbach's alpha), reliability, and measurement error. Validity is "the 
degree to which an instrument measures the construct(s) it purports to measure" with the measurement properties: content, construct, and criterion validity. Content validity is described as the degree to which the content of an instrument is an adequate reflection of the construct to be measured and includes face validity (91). Face validity is a subjective assessment if the measurement instrument appears to be an adequate reflection of the construct, this is done by simply looking at the questions. Construct validity is described as the degree to which scores of an instrument are consistent with hypotheses, e.g., with regards to internal relationships (often measured with factor analysis), relationships with scores of other instruments or differences between relevant groups. Criterion validity is described as the degree to which scores of an instrument are an adequate reflection of a gold standard. Finally, responsiveness is the ability of an instrument to detect changes over time (91).

\section{BetterBack@; model of care}

\section{Development of the BetterBack:-) MoC}

The implementation process started in 2016, when primary care physiotherapy managers expressed a need for improved PT delivered LBP management. Their arguments were justified by the high prevalence and burden of LBP $(7,92)$ together with a perceived fragmented LBP care, the use of PT intervention not in line with evidence-based international guidelines (3), and the lack of Swedish best practice clinical guidelines. The current routine care clinical pathway for patients with LBP before implementation of the BetterBack $\odot$ is outlined in Figure 4. 


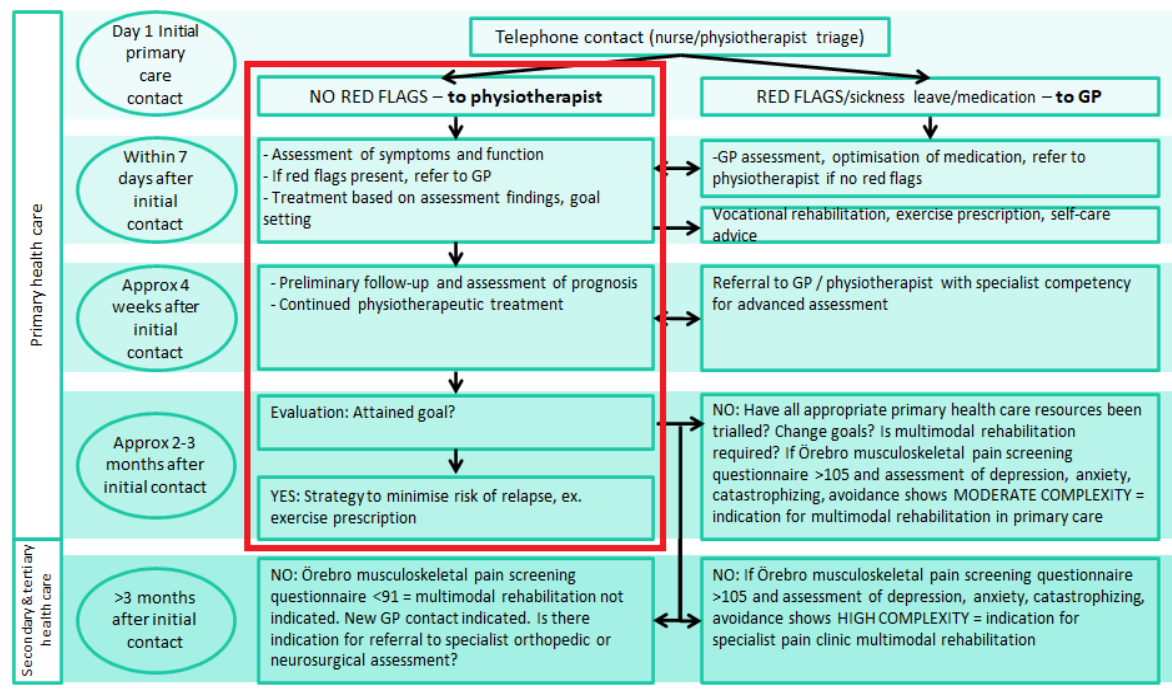

Figure 4. Current routine care clinical pathway for patients LBP in the healthcare region of Östergötland before implementation. The red square is the focus area for the implementation of the BetterBack $;$ - MoC. GP $=$ General Practitioner, LBP $=$ Low Back Pain, $\mathrm{MoC}=$ Model of Care

To guide the development of the BetterBack $:$ MoC, an international musculoskeletal framework was used. It contained checklists to assist in developing, implementing and evaluation phases (75). A multifaceted implementation strategy was used, with the aim of targeting different organisational levels (93). A top-down strategy started the process with a request and support from the rehabilitation managers in an existing implementation forum. This implementation forum is a group of all rehabilitation managers from the three rehabilitation units and clinical researchers; its purpose is to facilitate the implementation process in different phases as regards timeline, logistics and goals (94). A senior researcher in the implementation forum, Birgitta Öberg (BÖ), initiated the development of the MoC. Secondly, two clinical researchers, Allan Abbott and Karin Schröder, along with 'clinical champions', were involved in a bottom-up strategy to develop and locally adapt two international LBP guidelines $(38,95,96)$ into the BetterBack $:)$ MoC. The six clinical champions were selected by the managers from each unit. These were trusted clinicians with special skills in LBP diagnosing and treatment. The clinical champions and the two clinical researchers formed the MoC support team. The development of the BetterBack $:-$ MoC followed six steps with the MoC support team involved in step all the steps: 
1. Discussion of the current routine care clinical pathway for LBP and areas needing improvement outlined by the red square in Figure 4.

2. Discussion and analysis of the existing international best practice clinical guidelines for LBP with or without radiculopathy by the English National Institute for Health and Care Excellence (The NICE guidelines) and by the Danish Health and Medicine Authority (38, 95, 96).

3. Local adaptation of the international best practice clinical guidelines to the Swedish context using the Swedish National Board of Health and Welfare methods for guideline construction (97). The clinical guideline recommendations were supported by a clinical priority ranking based on overall grade of evidence together with a consensus based on professional experience and patient benefits versus harms and costs (98). The clinical practice guideline recommendations with their priority ranking as well as support tools used in the BetterBack(-) MoC can be found in Appendix 1.

Identification and discussion of PTs' target behaviours of relevance for the BetterBack - MoC and its modifiable barriers for uptake. Support tools for LBP patient management were developed; the aim was to further educate and enable PTs' clinical reasoning in providing LBP assessment and treatment according to the Swedish adaptation of the best practice clinical guidelines. A total of 13 learning goals were identified, and these were linked to learning activities with different behavioural change techniques (Table 1 in Paper II). The behaviour change wheel (BCW) $(67,72)$ was used as a logic model to theorise the process of designing the BetterBack $-:$ MoC (Figure 3).

4. Developing of a multifaceted implementation strategy (described in the method section).

5. Once behaviour change among PTs has occurred; it was expected that PTs' use of the BetterBack:-) MoC may influence patient outcomes. The rationale for this can be based on the common sense model of self-regulation (CSM) (99). This suggests a potential effect of the BetterBack $\odot$ MoC on improved patient-reported outcomes measuring disability, function, pain, patient illness beliefs and coping and self-enablement strategies.

\section{Content of the BetterBack:- MoC}

The content of the BetterBack $\odot$ MoC aims to change patients' maladaptive illness beliefs, low expectation of recovery, fear avoidance, anxiety and catastrophising, low self-care enablement and low physical activity level. We hypothesised that increased ability of being physically 
active and improved illness beliefs lead to increased perceived physical capacity, reduced fear avoidance and improved expectations. These changes were then hypothesised to translate to an increase of patients' selfefficacy and self-management strategies, which in turn was expected to improve PROMs.

The content that differs from routine practice includes the following support tools linked to each guideline recommendations (Appendix 1): evidence-based guideline recommendations; patient centred coordinated care pathways (Figure 5); structured assessment proformas; SBT (33); clinical process and reasoning pathway flowchart; clinical reasoning and process evaluation tool (CRPE); standardised patient brochure and a powerpoint with additional material for group-based patient education; and exercise resources.

The education/information parts addressed these components: The good prognosis of LBP, this to reduce patient's negative thoughts and beliefs about LBP. Pain neuroscience and information about back pain, this to enable patients to understand the complexity of pain perception with involvement of thoughts and beliefs. Advice to handle flare ups with the aim to improve patient's understanding and pain acceptance and to improve patient's sense of control and self-efficacy. The natural cause of back pain and that LBP is a recurrent condition where medical imaging generally is not recommended because of unspecific findings, this with the aim to reduce patient's expectation of finding the exact source of pain. Education about physical activity and encouragement to stay physically active, this with the aim to reduce patient's fear avoidance, improve pain control and improve physical fitness. Furthermore, standardised tools to support the design and progression of home-based and/or group-based exercise program with the aim to improve patient's function, muscle strength, flexibility and physical fitness.

Steps involved in the content delivery are outlined in Figure 5:

1) Patients where first assessed using structured proformas, and the SBT. The use of SBT allowed PTs to use the SBT approach, with matching patients to treatment packages appropriate for them, based on prognosis (39). Less treatment with few visits was therefore recommended for patients in the low-risk group, and more treatment with additional training, professional support to address barriers to recovery with more use of information/education with a behavioural medicine approach in higher risk groups.

2) Based on the assessment findings, the patient's functional impairments, activity limitations and ICD-10-SE (Swedish international classification of 
diseases and related health problems), diagnoses were registered with the Clinical Reasoning and Process Evaluation tool (CRPE).

3) Treatment was matched to identified functional impairments and activity limitations. All patients received individual information about the findings in the assessment. Other treatment options were mobilisation techniques, individualised training, and patient education with a brochure. The intervention delivery and dosage were based on the PTs' clinical reasoning regarding patients' risk of developing persistent pain and towards patients' goals. After step 3, patients received a follow-up assessment and evaluation of treatment outcomes, and if needed receive 4) group-based care (BetterBack $;$; part 2) with continued education on backpain with a biopsychosocial approach delivered in one 90-minute session with 2-10 participants and/or group training for six weeks twice a week with a recommended 12-week intervention period. Choice of exercise, dosage and progression was done by the supervising PT together with the patient. The group-based intervention could be done by another PT than the assessment PT, however follow-ups were always performed by the assessment PT. Appendix 1 shows the content in detail.

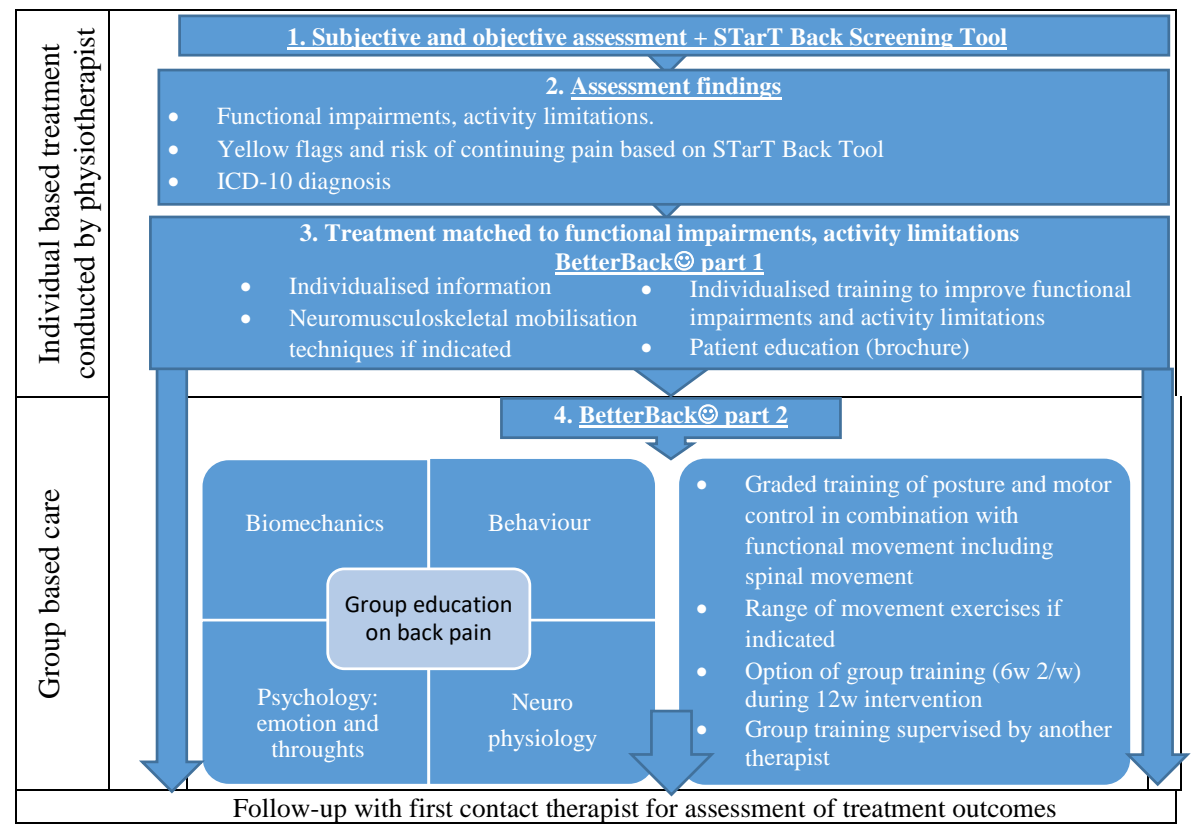

Figure 5. Patient centred coordinated care pathways with content of the BetterBack $\odot$ MoC. 


\section{Rationale of the thesis}

In Sweden and in many other countries, most patients with LBP are treated in primary care, and often by PTs. However in Sweden a formal clinical guideline for the treatment of LBP was still lacking. Furthermore, there are interventions that Swedish PTs in primary care consider relevant in clinical practice despite absence of consensus about effects or evidence, which results in non-evidence-based treatment with non-optimal care (3). There was also a call from rehabilitation managers from publicly funded financed healthcare to improve LBP care in the region, which they perceived as fragmented and not always in line with evidence. Therefore, implementation of an LBP model of care was needed with evaluation of both clinicians and patient outcomes. There is also a need for more valid, feasible and adapted instruments to measure determinants of healthcare practitioners' implementation behaviour, in order to understand success or failure in LBP guideline implementation. This thesis, with its robust design, can facilitate the integration of evidence-based practice into primary care physiotherapy, with the aim of improving LBP quality of care and patient outcomes. 
Implementing BetterBack - a Best Practice Physiotherapy Healthcare Model for Low Back Pain 


\section{AIMS OF THE THESIS}

\section{Overall aim}

The overall aim of this thesis was to evaluate a multifaceted implementation strategy and a best practice physiotherapy healthcare model for LBP.

\section{Specific aims}

- To tailor the Determinants of Implementation Behaviour Questionnaire (DIBQ) for evaluation of clinician expectations regarding the implementation of best practice LBP primary care programs in Sweden and Denmark (Paper I).

- To describe and evaluate determinants of implementation behaviour among PTs after implementation of the BetterBack:-) MoC (Paper II).

- To evaluate and compare PTs' confidence, attitudes and beliefs in managing patients with LBP before with after implementation of the BetterBack:) MoC (Paper II).

- To evaluate and compare PTs' adherence to clinical practice guideline recommendations for LBP after implementation of the BetterBack $:$ MoC with routine care (control) (Paper III).

- To evaluate and compare patient-reported outcomes after implementation of the BetterBack $\odot$ MoC with routine care (control) (Paper IV).

- To evaluate and compare patient-reported outcomes based on the fidelity of clinical practice quality index (CPQI) adherence regarding PTs' care (Paper IV). 
Implementing BetterBack - a Best Practice Physiotherapy Healthcare Model for Low Back Pain 


\section{METHOD}

\section{Designs}

An overview of the studies is presented in Table 1. The four papers in this thesis are based on one methodological study (Study A) and on a series of experimental studies packaged in a hybrid type II trial (Study B) (Papers II-IV).

Table 1. Overview of the four papers included in this thesis

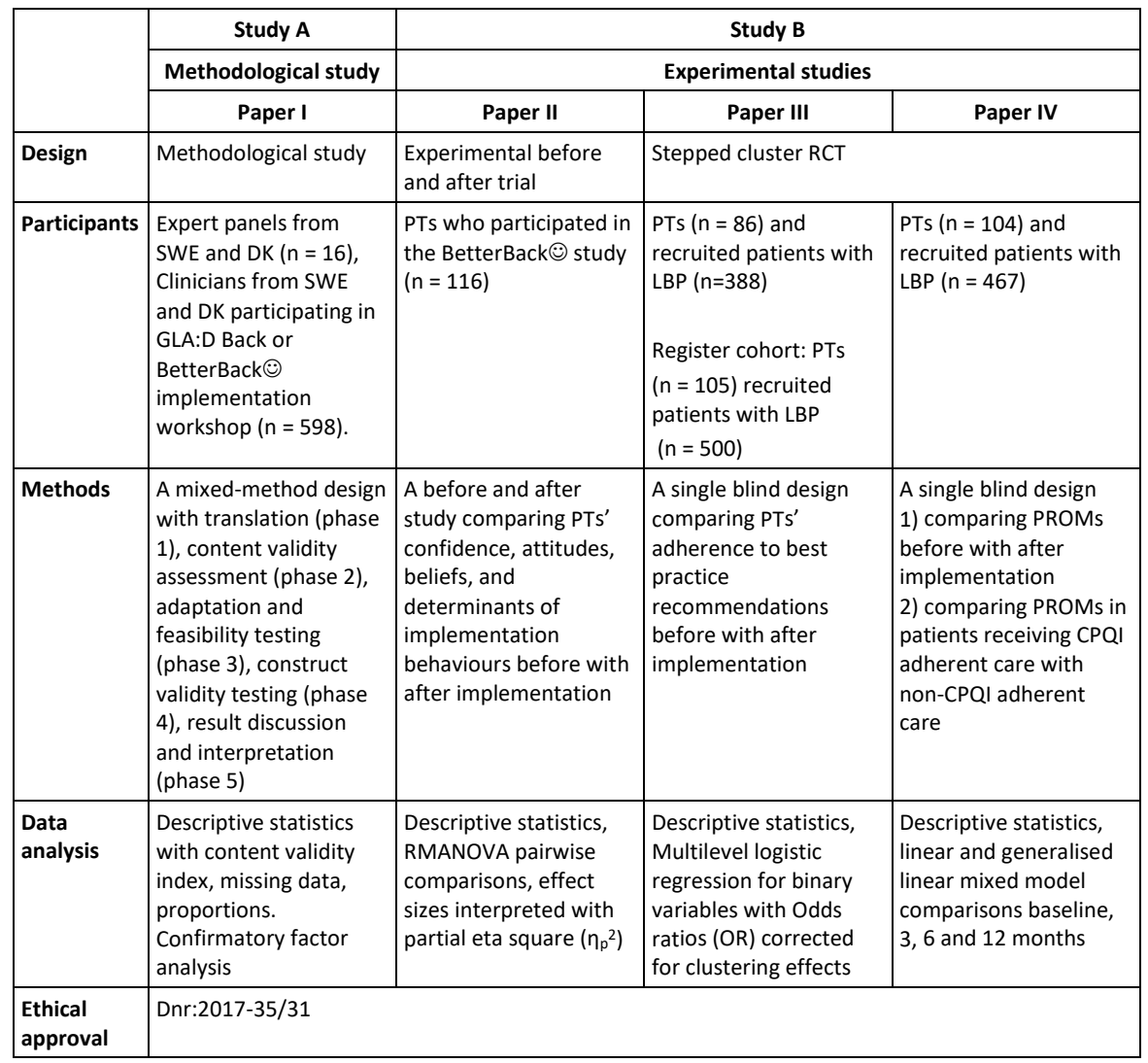

$\mathrm{RCT}=$ Randomised Controlled Trial, SWE $=$ Sweden, $\mathrm{DK}=$ Denmark, $\mathrm{n}=$ number of participants, $\mathrm{PT}=$ physiotherapist, PROMs = Patient-reported Outcome Measures, LBP = Low Back Pain, RMANOVA = Repeated Measures Analysis of Variance, $\mathrm{CPQI}=$ Clinical Practice Quality Index 


\section{Settings and participants}

All studies have been conducted in the publicly funded primary care physiotherapy clinics in the healthcare region of Östergötland, Sweden. Additionally, in Study A, academics from Linköping University were involved in the expert panel along with clinicians and academics from Denmark. In Study B, PTs came from the three organisational clusters within the healthcare region and patients were nested in these three clusters based on the existing geographical and organisational structure outlined in Figure 6.

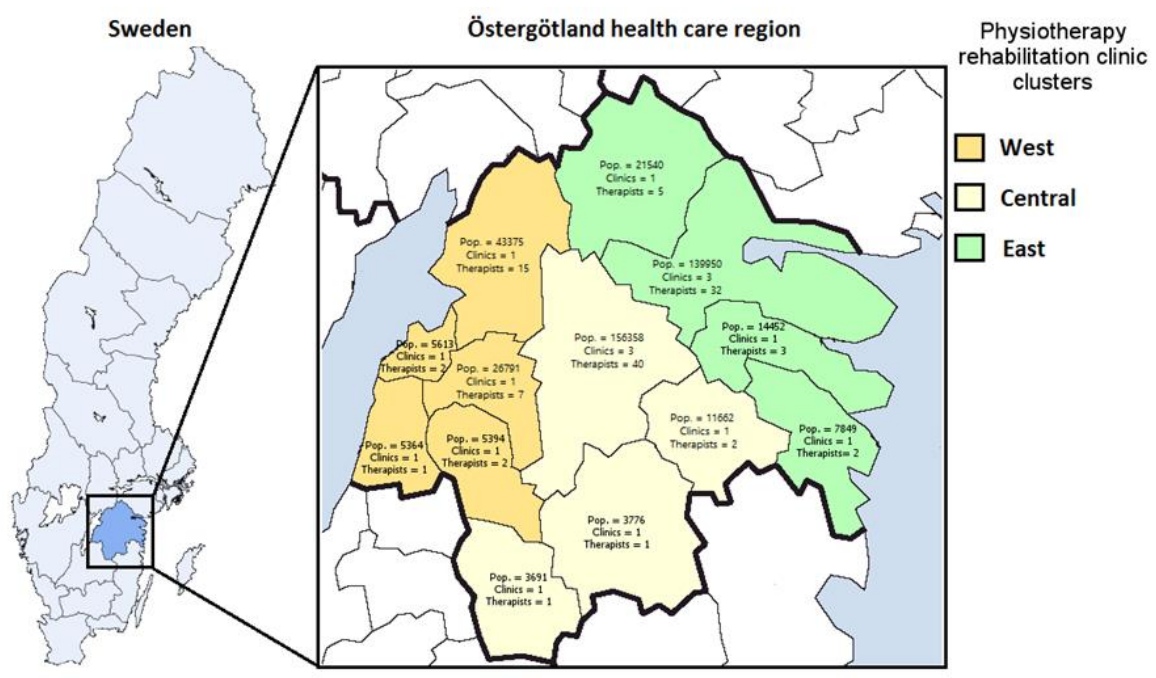

Figure 6. Municipal resident population and number of physiotherapy rehabilitation clinics and physiotherapists in the west, central and east cluster in Östergötland healthcare region year 2016

In Papers III-IV a stepped cluster randomised control trial (RCT) design was used as outlined in Table 2. 
Table 2. The stepped structure (dog leg design) of the cluster randomised controlled trial

\begin{tabular}{|c|c|c|c|c|c|c|c|c|c|c|c|c|}
\hline \multicolumn{10}{|c|}{2017} & \multicolumn{3}{|c|}{2018} \\
\hline Mar & Apr & May & Jun & Jul & Aug & Sep & Oct & Nov & Dec & Jan & Feb & Mar \\
\hline $\begin{array}{l}\text { PT Cluster } \\
\text { random } \\
\text { allocation }\end{array}$ & \multicolumn{2}{|c|}{$\begin{array}{l}\text { Patient recruitment } \\
\text { during internal pilot } \\
\text { phase }\end{array}$} & \multicolumn{10}{|c|}{ Patient recruitment during main trial phase } \\
\hline $\begin{array}{l}\text { Cluster } 1 \\
\text { West }\end{array}$ & MoC & $\mathrm{MoC}$ & $\mathrm{MoC}$ & MoC & MoC & $\mathrm{MoC}$ & MoC & $\mathrm{MoC}$ & $\mathrm{MoC}$ & $\mathrm{MoC}$ & MoC & $\mathrm{MoC}$ \\
\hline \multicolumn{13}{|c|}{ MoC implementation } \\
\hline \multirow{2}{*}{$\begin{array}{l}\text { Cluster } 2 \\
\text { Central }\end{array}$} & \multirow[t]{2}{*}{ Routine } & \multirow[t]{2}{*}{ Routine } & \multirow[t]{2}{*}{ Routine } & \multirow[t]{2}{*}{ Routine } & Routine & $\mathrm{MoC}$ & MoC & \multirow[t]{2}{*}{ MoC } & \multirow[t]{2}{*}{ MoC } & \multirow[t]{2}{*}{$\mathrm{MoC}$} & \multirow[t]{2}{*}{$\mathrm{MoC}$} & \multirow[t]{2}{*}{ MoC } \\
\hline & & & & & MoC imp & lementati & & & & & & \\
\hline $\begin{array}{l}\text { Cluster } 3 \\
\text { East }\end{array}$ & Routine & Routine & Routine & Routine & Routine & Routine & Routine & Routine & Routine & Routine & Routine & Routine \\
\hline
\end{tabular}

PT $=$ physiotherapist, $\mathrm{MoC}$ model of care $=$ intervention group, Routine $=$ control group

\section{Inclusion criteria for expert panel and clinicians}

In Study A participants came from both Sweden and Denmark. The inclusion criteria for the expert panel were experts with methodological research background in the implementation and/or musculoskeletal fields. Demographics of the expert panel are outlined in Table 3. The inclusion criteria for clinicians were clinicians who had participated in the Swedish BetterBack () (all publicly funded primary care rehabilitation clinics in the Östergötland healthcare region of Sweden) or in the Danish GLA:D® Back (snowball sampled PTs and chiropractors) two-day workshop.

Table 3. Demographics of expert panel

\begin{tabular}{lccc}
\hline Variables & Sweden $(\mathbf{n}=\mathbf{7})$ & Denmark ( $\mathbf{~ = ~ 9 ) ~}$ & Total (n = 16) \\
\hline Male / female, $\mathrm{n}$ & $1 / 6$ & $3 / 6$ & $4 / 12$ \\
$\begin{array}{l}\text { Physiotherapist / chiropractor / } \\
\text { other, } \mathrm{n}\end{array}$ & $3 / 0 / 4$ & $4 / 5 / 0$ & $7 / 5 / 4$ \\
$\begin{array}{l}\text { Age, mean (SD) } \\
\text { Years of clinical experience, }\end{array}$ & $55.4(7.6)$ & $44.9(11.5)$ & $49.5(11.1)$ \\
$\begin{array}{l}\text { mean (SD) } \\
\text { Years of research experience, } \\
\text { mean (SD) }\end{array}$ & $14.7(10.6)$ & $15.6(12.5)$ & $15.2(11.3)$ \\
\hline
\end{tabular}

SD $=$ Standard Deviation

Inclusion criteria for Study A and B were PTs regularly working with patients with LBP in all primary care rehabilitation clinics in the Östergötland public healthcare region of Sweden. In Study A, also PTs and chiropractors from Denmark were involved. Clinician demographics are displayed in Table 4 . 
Table 4. Demographics of clinicians in Studies I-IV

\begin{tabular}{|c|c|c|c|c|c|}
\hline \multirow[t]{2}{*}{ Variables } & \multirow{2}{*}{$\begin{array}{c}\text { Paper I } \\
(n=598)\end{array}$} & \multirow{2}{*}{$\begin{array}{l}\text { Paper II } \\
(n=116)\end{array}$} & \multicolumn{2}{|c|}{ Paper III } & \multirow{2}{*}{$\begin{array}{l}\text { Paper IV } \\
(\mathrm{n}=104)\end{array}$} \\
\hline & & & $(n=86)^{1}$ & $(n=105)^{2}$ & \\
\hline Sex, female, n (\%) & $226(39)$ & $82(71)$ & $59(69)$ & $74(70)$ & $73(70)$ \\
\hline Age, mean (SD) & $39(11)$ & $38(12)$ & $36(11)$ & $37(11)$ & $37(11)$ \\
\hline $\begin{array}{l}\text { Physiotherapist / } \\
\text { chiropractor, } \mathrm{n}\end{array}$ & $550 / 48$ & $116 / 0$ & $86 / 0$ & $105 / 0$ & $104 / 0$ \\
\hline \multicolumn{6}{|l|}{ Clinical experience, $\mathrm{n}(\%)$} \\
\hline $1-5$ years & $197(33)$ & $53(46)$ & $44(52)$ & $50(50)$ & $49(49)$ \\
\hline $6-10$ years & $103(17)$ & $21(18)$ & $13(16)$ & $16(16)$ & $16(16)$ \\
\hline $11-15$ years & $89(15)$ & $10(9)$ & $6(7)$ & $10(10)$ & $10(10)$ \\
\hline $16-20$ years & $81(14)$ & $9(8)$ & $8(10)$ & $8(8)$ & $8(8)$ \\
\hline$>20$ years & $123(21)$ & $23(20)$ & $13(15)$ & $17(17)$ & $17(17)$ \\
\hline \multicolumn{6}{|l|}{ Education level, n (\%) } \\
\hline Bachelor's degree & & $100(87)$ & $72(86)$ & $85(87)$ & $84(87)$ \\
\hline Post graduate major & & $8(7)$ & $8(10)$ & $8(8)$ & $8(8)$ \\
\hline Clinical specialist* & & $4(3)$ & $4(5)$ & $4(4)$ & $4(4)$ \\
\hline Post graduate master & & $3(3)$ & $3(4)$ & $1(1)$ & $1(1)$ \\
\hline
\end{tabular}

${ }^{1}$ Data from physiotherapist Clinical Reasoning and Process Evaluation tool, ${ }^{2}$ Register data, $\mathrm{SD}=$ Standard Deviation, ${ }^{*}$ Clinical specialist by the Swedish association of registered physiotherapy

\section{Inclusion criteria for patients}

In the experimental studies that apply a stepped randomised controlled trial design (Papers III-IV), the inclusion criteria for patients were 18-65 years, fluent in Swedish and accessing public primary care due to a firsttime or recurrent episode of acute, subacute, or chronic phase benign LBP with or without radiculopathy. Exclusion criteria were current diagnosis of a malignancy, previous malignancy in the last five years, infection, spinal fracture, cauda equina syndrome, spinal surgery in the last two years, current pregnancy, or previous pregnancy up to three months, ankylosing spondylitis or rheumatic disease, participants who fulfill the criteria for multi-modal/multiprofessional rehabilitation for complex long-standing pain and severe psychiatric diagnosis. Demographics are displayed in Table 5 . 
Table 5. Demographics of patients in the stepped randomised controlled trial (Papers III-IV)

\begin{tabular}{|c|c|c|}
\hline Variables & Paper III $(n=500)$ & Paper IV $(n=467)$ \\
\hline Sex, female, n (\%) & $279(56)$ & $261(56)$ \\
\hline Age, mean (SD) & $45(12)$ & $45(12)$ \\
\hline \multicolumn{3}{|l|}{ Educational level, n (\%) } \\
\hline Elementary & \multicolumn{2}{|c|}{$58(13)$} \\
\hline High school & \multicolumn{2}{|c|}{$270(58)$} \\
\hline University & \multicolumn{2}{|c|}{$137(30)$} \\
\hline \multicolumn{3}{|l|}{ Pain Duration, $\mathrm{n}(\%)$} \\
\hline$<12$ weeks & \multicolumn{2}{|c|}{$269(58)$} \\
\hline$>12$ weeks & \multicolumn{2}{|c|}{$198(42)$} \\
\hline Employed, n (\%) & \multicolumn{2}{|c|}{$381(82)$} \\
\hline Sick leave due to back pain, $\mathrm{n}(\%)$ & \multicolumn{2}{|c|}{$82(19)$} \\
\hline NRS back pain, mean (SD) & \multicolumn{2}{|c|}{$6.3(2.2)$} \\
\hline NRS leg pain, mean (SD) & \multicolumn{2}{|c|}{$3.7(3.3)$} \\
\hline ODI, mean (SD) & \multicolumn{2}{|c|}{$31(16)$} \\
\hline EQ-5D index, mean (SD) & \multicolumn{2}{|c|}{$0.535(0.301)$} \\
\hline General health, mean (SD) & \multicolumn{2}{|c|}{$6.4(2.1)$} \\
\hline \multicolumn{3}{|l|}{ SBT risk groups, $\mathrm{n}(\%)$} \\
\hline Low-risk group & \multicolumn{2}{|c|}{$172(37)$} \\
\hline Medium-risk group & \multicolumn{2}{|c|}{$234(50)$} \\
\hline High-risk group & \multicolumn{2}{|c|}{$61(13)$} \\
\hline
\end{tabular}

SD $=$ Standard Deviation

\section{Measurements}

Measurements and source of data are displayed in Table 6.

\section{Evaluation of the implementation intervention}

\section{Practitioner Self-Confidence Scale (PCS)}

PCS contains four items producing a total score of 20, where 4 represents the greatest self-confidence and 20 represents the lowest selfconfidence in diagnosing and clinical management of patients with LBP (100). PCS has shown an acceptable range of reliability with a Cronbach's coefficient above 0.70 (100).

Pain Attitudes and Beliefs Scale for Physiotherapists (PABS-PT)

PABS-PT consists of two domains, a biomedical orientation domain PABS-BM (score 10-60) and a biopsychosocial orientation domain PABS- 
BPS (score 9-54) (101). Higher scores represent higher biopsychosocial/biomedical orientation among PTs. The PABS-PT is the most used and thoroughly tested measure of attitudes and beliefs in physiotherapy research and the PABS-PT has shown acceptable construct and content validity $(101,102)$.

\section{Determinants of Implementation Behaviour Questionnaire (DIBQ)}

DIBQ evaluates healthcare professionals' potential determinants of implementation behaviours. It is used to understand barriers and facilitators for implementation success, in order to develop strategies to improve healthcare professionals' implementation behaviours (103). The questionnaire is based on the TDF (69) and BCW frameworks (68). The English original version with 93 items and 18 domains has good construct validity, and most domains showed good reliability, internal consistency and discriminant validity $(103,104)$.

\section{Clinical Reasoning and Process Evaluation tool (CRPE)}

CRPE enables analysis of PTs treatment protocols. It is based on the International Classification of Functioning, disability, and health (ICF) brief core set for LBP (105). The CRPE contains PT-reported choice of treatment for each patient during first and last consultation during the PT treatment period. The CRPE data formed the clinical practice quality index.

\section{Register data}

Register data from the public healthcare regional registry (PHRR) for each patient with number of PT treatments, referral to specialist consultation and medical imaging during PT period was collected from the public healthcare regional registry and used to evaluate adherence to CPGs. This data was also used in the clinical practice quality index.

\section{Evaluation of the clinical intervention}

\section{STarT Back Screening Tool (SBT)}

SBT is a nine-item questionnaire with questions relating to modifiable physical (items 1-4) and psychosocial risk factors (items 5-9) for long-term disabling back pain (33). The SBT produces three risk groups, which classify patients into low, medium, and high risk for poor LBP disability outcomes. The SBT produces an overall score between 0-9 and with a psychosocial subscale score (between 0-5) from items 5-9. A total score of $\leq 3$ points indicates low-risk group, $\geq 4$ points in combination with $<4$ 
points on the psychosocial subscale indicates medium-risk group, and a psychosocial subscale score of $\geq 4$ points indicate high-risk group (33). Apart from risk stratification to predict long-term disabling back pain, SBT can be used to match individuals to different care levels (39).

\section{Oswestry Disability Index (ODI)}

ODI evaluates back-pain-related function and activity limitations, reported as a score of $0-100 \%$ disability (106). It contains ten items, each item with six answer options, $0-5$ where higher values represent greater disability. ODI has been recommended as a core outcome by international consensus for clinical trials in non-specific LBP. Furthermore, ODI has shown good construct validity and reliability (107). ODI together with Numeric rating scale (NRS) for pain intensity and health-related quality of life with EQ 5-D index have been suggested by international consensus to be used as standardised outcomes to evaluate LBP management (108).

\section{Numeric Rating Scale (NRS-LBP)}

NRS-LBP evaluates low back pain intensity during the past week in an 11-point scale ranges from $0-10$, where $o$ represents 'no pain' and 10 represents 'worst imaginable pain'. NRS has adequate validity (109).

\section{European Quality of Life Questionnaire (EQ-5D)}

EQ-5D evaluates generic health-related quality of life and is computed into an index from 1 (highest health-related quality of life) to -0.59 (lowest health-related quality of life) (110). The EQ-5D is one of the most widely used generic questionnaires evaluating treatment success for CLBP (111).

\section{Brief Illness Perception Questionnaire (BIPQ)}

BIPQ evaluates cognitive illness representation. Eight items produce a score ranging from $0-80$, representing the degree to which the LBP is perceived as benign or threating. Higher scores indicate more threatening views of the illness (112). A review and meta-analysis has concluded that BIPQ is widely used and has good psychometric properties (113).

\section{Patient Enablement Instrument (PEI)}

PEI evaluates patient self-care enablement using six items. Answers are rated on a three-point scale ranging from 0-2 points, producing a score from 0-12, where a higher score reflects higher self-care enablement (114). The Swedish version of the PEI has shown high internal consistency and moderate to good reliability for patients in a primary care setting (115). PEI is a transition rating score and used only at 3-, 6- and 12-month follow-ups. 


\section{Patient Satisfaction (PS)}

PS evaluates patient satisfaction with their LBP care, using a single item question: "How satisfied are you with the results of your LBP treatment" (116). The five response options are satisfied (1), somewhat satisfied (2), neither satisfied nor dissatisfied (3), somewhat dissatisfied (4), very dissatisfied (5). The scale was dichotomized into satisfied $=1$ and 2 , dissatisfied $=3$ to 5 . This single item is included in the Swedish six item Treatment Outcome Satisfaction Questionnaire (TOSQ-S) (117). TOSQ-S has shown acceptable psychometric properties (117). PS is a transition rating score and used only at 3-, 6- and 12-months follow-ups.

\section{Patient Global Impression of Change (PGIC)}

PGIC evaluates how patients perceive that their LBP has changed during their time in the study, from the beginning of treatment to the present (118). The question used was 'How is your LBP today compared to before treatment?'. PGIC is a balanced 11-point scale with a midpoint unchanged ranging from -5 (very much worse) through o (unchanged) to 5 (completely recovered), and this version with its bipolar construct is suggested as the best scale for measuring global rating of change (118), with good patient preference and test-retest reliability (119). 
Table 6. Description of measurements used in Papers I-IV

\begin{tabular}{|c|c|c|c|c|c|c|}
\hline Measurements & & $\begin{array}{c}\text { Data } \\
\text { source }\end{array}$ & Paper I & Paper II & $\begin{array}{l}\text { Paper } \\
\text { III }\end{array}$ & $\begin{array}{l}\text { Paper } \\
\text { IV }\end{array}$ \\
\hline \multicolumn{7}{|l|}{$\begin{array}{l}\text { Evaluation of Implementation } \\
\text { intervention }\end{array}$} \\
\hline $\begin{array}{l}\text { Determinants of implementation } \\
\text { behaviours }\end{array}$ & DIBQ & $\mathrm{CR}$ & $x$ & $x$ & & \\
\hline Treatment orientation & PABS-PT & $C R$ & & $x$ & & \\
\hline Practitioners' confidence & PCS & $\mathrm{CR}$ & & $x$ & & \\
\hline Guideline adherence & CRPE & CR & & & $x$ & $x$ \\
\hline Number of PT treatments & \multicolumn{2}{|c|}{ PHRR } & & & & $x$ \\
\hline Referral to specialist consultation & \multicolumn{2}{|c|}{ PHRR } & & & $x$ & $x$ \\
\hline Medical imaging & \multicolumn{2}{|c|}{ PHRR } & & & $x$ & $x$ \\
\hline \multicolumn{7}{|l|}{ Evaluation of clinical intervention } \\
\hline Risk stratification & SBT & PR & & & $x$ & \\
\hline Disability & ODI & PR & & & & $x$ \\
\hline Pain intensity & NRS & PR & & & & $x$ \\
\hline Health-related quality of life & $E Q-5 D$ & PR & & & & $x$ \\
\hline Illness perception & $\mathrm{BIPQ}$ & PR & & & & $x$ \\
\hline Patient enablement & PEI & PR & & & & $x$ \\
\hline Patient satisfaction & PS & PR & & & & $x$ \\
\hline Perceived change in LBP disability & PGIC & PR & & & & $x$ \\
\hline
\end{tabular}

$\mathrm{DIBQ}=$ Determinants of Implementation Behaviour Questionnaire, CPRE = Clinical Reasoning Process Evaluation tool, PHCRR = Public Healthcare Regional Registry, $C R=$ Clinician Reported, $P R=$ Patient Reported

\section{Intervention}

\section{The BetterBack $\odot$ MoC}

The BetterBack $:$ MoC development and content is briefly described in the thesis background, and in the protocol (76). A sustained multifaceted implementation strategy was used to overcome the modifiable barriers facilitated by clinical champions and researchers in an MoC support team and the implementation forum. The managers supported the implementation by making participation to the workshop mandatory for all PTs and by organising the workshop on two alternative occasions in each setting. The clinical champions in the MoC support team acted as clinical ambassadors and local facilitators at their rehabilitation unit. To support sustainability, the clinical champions educated new staff and were the clinic contact person throughout the study period. The MoC support team designed the two-day (13.5 hours) mandatory workshop in the three geographical units of public primary care rehabilitation clinics in 
Östergötland. The key components of the workshop education described in the protocol (76) were: persuasion and education about evidence-based recommendations for LBP and the content and use of BetterBack:) MoC. The implementation process was introduced and followed up with one to two outreach visits by the two clinical researchers to the participating clinics. To facilitate communication between participating PTs and researchers, a webpage with a chat function, support tools and guideline recommendations was available. The following is a description of the BetterBack $\odot$ MoC content summarised according to the TIDieR template (Template for intervention description and replication) (120).

- WHY: The main PT target behaviour was the adoption of the BetterBack $\odot$ MoC to influence PT delivered care coherent with best practice guideline recommendations.

- WHAT: This would require the content of the BetterBack -$)$ MoC to change barrier behaviours such as low confidence in skills/capabilities for improving LBP patient management, low awareness of the model, beliefs of the negative consequences of the $\mathrm{MoC}$ and a biomedical treatment orientation rather than a biopsychosocial orientation (48).

- HOW: BetterBack $\odot$ MoC content used to overcome modifiable barriers includes support tools aimed to further educate and enable PTs' clinical reasoning in providing assessment and treatment coherent with the Swedish adaptation of best practice LBP guidelines. These support tools are described in the background section and in Appendix 1.

- WHEN/HOW MUCH/TAILORING: Intervention delivery, frequency, dosing and progression were stratified based on PTs' clinical reasoning regarding risk of pain persistence and towards patient goals.

- PROCEDURE: The procedure for delivering the BetterBack@: MoC is displayed in Figure 5 and in the background section under the heading "Content of the BetterBack $\odot$ MoC". 


\section{Data analyses and methodological considerations}

Statistical analyses applied in all papers are displayed in Table 7.

Table 7. Statistical analyses applied in Papers I-IV

\begin{tabular}{lcccc}
\hline Statistical analysis & Paper I & Paper II & Paper III & Paper IV \\
\hline Descriptive statistics & $\mathrm{X}$ & $\mathrm{X}$ & $\mathrm{X}$ & $\mathrm{X}$ \\
Content validity index (CVI) & $\mathrm{X}$ & & & \\
Confirmatory factor analysis & $\mathrm{X}$ & & & \\
Effect size, partial eta square & & $\mathrm{X}$ & $\mathrm{X}$ & $\mathrm{X}$ \\
Repeated Measures Analysis of Variance (RMANOVA) & & & $\mathrm{X}$ \\
Multi-level logistic regression & & & $\mathrm{X}$ \\
Linear mixed model regression & & & $\mathrm{X}$ \\
Generalised mixed model regression & & & $\mathrm{X}$ \\
Minimal clinically important change (MCID) & & & \\
Optimal cut-off point (OPC) & & & \\
\hline
\end{tabular}

\section{Questionnaire development (Study A, Paper I)}

A mixed methods design was used as outlined in Figure 7. First was a forward translation of the original 94 item DIBQ, then adaptation of the instrument using content validity assessment into a tailored version (DIBQ-t). This tailored version was first feasibility-tested and then construct-validity-tested using confirmatory factor analysis with data from clinicians from Denmark and Sweden. Finally, the result of the DIBQ-t was mapped to the COM-B model.

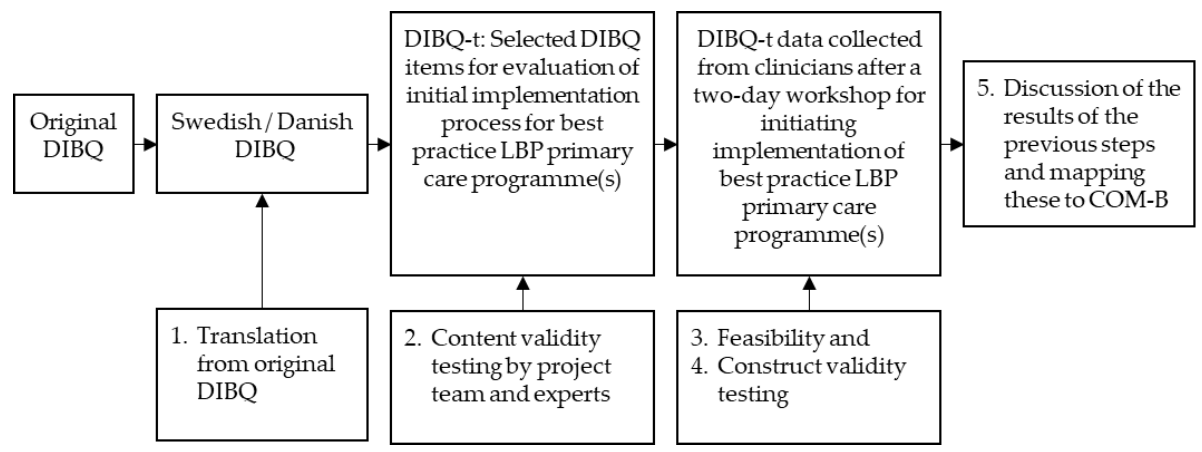

Figure 7. The mixed method design for Study A

Expert panel and clinician demographics are presented in Tables 3 and 4 as means with standard deviation (SD) and proportions (\%). Translation of the original DIBQ was performed according to the guideline by Beaton 
(121) using a panel of experts in implementation research and musculoskeletal health (122). Content validity was first assessed by the project team through selecting relevant and realistic number of items from the original DIBQ.

Thereafter, experts in the Swedish and Danish expert panels were asked to rate all DIBQ items on a 1-4 Likert scale ranging from "not relevant" to "very relevant". These ratings were indexed using a Content Validity Index (CVI), and this was analysed and reported descriptively (123). To consider an item 'relevant', $80 \%$ or more of the experts had to rate the question as 'relevant' or 'very relevant' (CVI $\geq 0.80$ ). Items included in the DIBQ-t were based on three criteria 1) selected by project leaders + experts rated CVI $\geq 0.80$ or 2 ) experts rated CVI 1.0o, irrespective of the selection by the project leaders, or 3) project leaders selected items which were rated with CVI $\geq 0.80$ country-wise to allow context differences between the two countries.

To determine feasibility in phase three, the proportion of missing data for specific items from clinicians from the two countries was used. Item and domain-level data were analysed as categorical data and reported as the proportion of clinicians responding to each response category. Ratings of 'agree' or 'strongly agree' were classified as positive expectations to implementation, whereas items and domains rated as 'neither nor', 'disagree' or 'strongly disagree' were classified as neutral/negative expectations to implementation. For items 5-9, response options were changed to 'very easy', 'easy', 'neither easy nor difficult'.

To assess the internal construct validity in phase four for the DIBQ- $t$ domains, a confirmatory factor analysis (124) with Laavan package in R version 3.5.1 was used. Standardized root mean squared residual (SRMR), root mean square error of approximation (RMSEA), (both with cut-off < o.05), comparative fit index (CFI) and Tucker-Lewis index (TLI) (both with cut-off score $>0.9$ ) were used to evaluate the model fit (125). In phase five the project group discussed and interpreted the results using the COM-B model.

\section{Confidence, attitudes, beliefs, and implementation behaviours (Study B, Paper II)}

This was a before and after experimental design involving PTs before and after implementation of the BetterBack-; MoC. It evaluated implementation outcomes such as change of attitudes and beliefs, confidence and determinants of implementation behaviours. In Table 4, PTs' baseline characteristics are presented as means with standard deviation (SD) and proportions (\%). In Figure 8 shows measurement time points for the different outcomes. An a-priori sample size calculation was performed for improvement of PCS directly after the workshop, as well as 
after 3 and 12 months compared to baseline based on a correlation between repeated measures $=0.5$, an $80 \%$ statistical power and a significance level of $\mathrm{p}=0.05$. A sample of 43 patients was needed, calculated with an estimated 20\% loss of follow-up for an RMANOVA, assuming at least a small Cohen's d effect of 0.2. PTs' confidence, attitudes and beliefs and determinants of implementation behaviour were evaluated longitudinally with RMANOVA. Main effect size and pairwise comparisons' effect sizes were interpreted with partial eta square $\left(\eta_{\mathrm{p}}{ }^{2}\right)$, where a small effect size is considered when $\eta_{p^{2}}=0.01$, medium effect size $\eta_{p^{2}}=0.06$ and large effect size $\eta_{\mathrm{p}}{ }^{2}=0.14$ (126). Multiple imputation was used to replace missing values based on values from baseline and actual time point. A chained equations procedure (fully conditional specification method in SPSS version 25, IMB Corporation, New York, USA) with 10 data sets was used in multiple imputation (127). For each variable, constraints were applied according to the range of the scale. A sensitivity analysis was performed where per protocol data without imputation was compared with intention to treat imputed data showing no substantial differences (128).

\section{workshop}

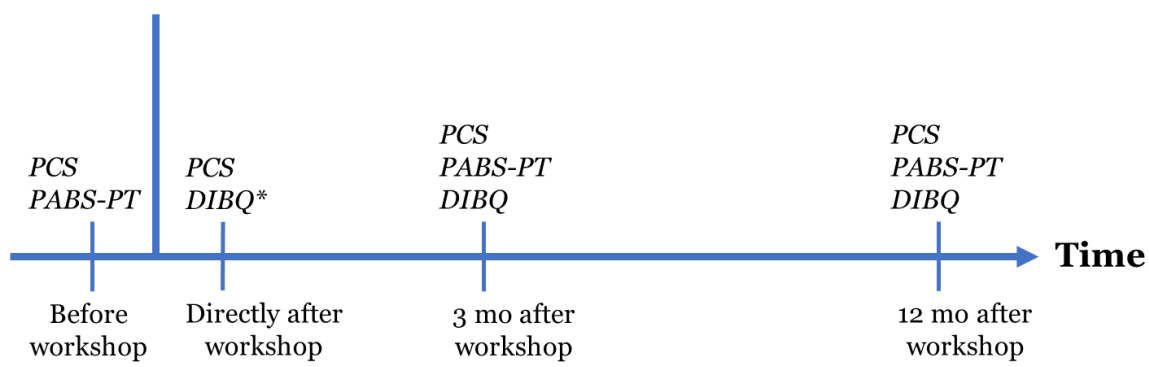

Figure 8. Measurement time points. PCS = Practitioner Self-Confidence Scale, PABS-PT = Pain Attitudes and Beliefs Scale for Physiotherapists, DIBQ = Determinants of Implementation Behaviour Questionnaire, mo $=$ months, ${ }^{*}$ Expected determinants: questions were rephrased to expected implementation behaviours since experiences at this time phase was lacking.

\section{Adherence to clinical practice guidelines (Study B, Paper III)}

This was a stepped, single-blind cluster RCT. PTs along with their patients were allocated to the BetterBack $\odot \mathrm{MoC}$ or routine care group according to cluster designation (Table 2 and Figure 6). The implementation outcome was PTs change of adherence to best practice recommendations. Descriptive statistics were used to compare independent variables between the two groups of patients and PTs at baseline, unpaired Student's t-test were used for continuous data and the Chi-Square test and Fishers Exact test for categorical data. Based on an a- 
priori hypothesised small-medium effect size difference from the trial protocol (76) and a previously reported $<50 \%$ CPG adherence in Swedish physiotherapy context (2) which has equivalence with an Odds ratio of 2 , a one-tailed p-value of 0.05 and $80 \%$ power 112 patient cases treated by the PTs were needed for logistic regression. Adherence to CPG recommendations were analysed by comparing the proportions of adherence in intervention versus control group. The clinical practice recommendations (with definitions) and target behaviour are presented in Table 8 . The primary outcome was referral to specialist consultation. In a secondary analysis, the highest clinical priority ranked CPG-related indicator variables (core recommendations) were merged and analysed using an overall, an assessment and a treatment clinical practice quality index (CPQI) (Table 9). Odds ratios (OR) with 95\% confidence intervals, pvalue and intracluster correlation coefficient (ICC) are presented from the multilevel logistic regression analyses for binary variables. Scores were corrected for clustering effects, and ICC was calculated according to the three regions that formed the two groups intervention and control group, with equal groups assumed. Sub-analyses in adherence according to SBT risk groups divided into low and medium/high risk were also performed. These SBT sub-analyses were used to analyse two target behaviours outlined in table $8 ; 1$ ) Fewer visits in the SBT low risk subgroup (1-3 visits) and more in the SBT medium/high-risk subgroup ( $\geq 4$ visits); 2) Increase overall use and stratified use of educational interventions with higher proportions in higher risk groups. The regression coefficients were transformed to odds ratios with 95\% confidence intervals (CI), indicating the effect of the intervention. The level of significance was 0.05. All statistical analysis were performed using SPSS statistical software (SPSS V25, IBM Corporation, New York, USA) and with the statistical package R 3.6.o (R Core Team, 2018) (https://cran.r-project.org/). 
Table 8. CPG assessment- and treatment-related quality indicator variables, definitions, source of data and target behaviours

\begin{tabular}{|c|c|c|c|}
\hline & $\begin{array}{l}\text { CPG-related indicator } \\
\text { variables }\end{array}$ & Definition & $\begin{array}{l}\text { CPG-related target } \\
\text { behaviour, Source of data }\end{array}$ \\
\hline \multirow[t]{2}{*}{$\begin{array}{l}\text { Assessment } \\
\text { interventions }\end{array}$} & $\begin{array}{l}\text { 1. Proportion of } \\
\text { patients receiving } \\
\text { referral to specialist } \\
\text { consultation }\end{array}$ & $\begin{array}{l}\text { Referral to pain clinic, } \\
\text { orthopaedic or } \\
\text { neurosurgical care during PT } \\
\text { treatment period }\end{array}$ & $\begin{array}{l}\text { Reduce referral for benign } \\
\text { non-complex LBP, Register } \\
\text { data }\end{array}$ \\
\hline & $\begin{array}{l}\text { 2. Proportion of } \\
\text { patients receiving } \\
\text { medical imaging }\end{array}$ & $\begin{array}{l}\text { X-ray, MRI, CT scan during } \\
\text { PT treatment period }\end{array}$ & $\begin{array}{l}\text { Reduce imaging for benign } \\
\text { non-complex LBP, Register } \\
\text { data }\end{array}$ \\
\hline \multirow[t]{6}{*}{$\begin{array}{l}\text { Treatment } \\
\text { interventions }\end{array}$} & $\begin{array}{l}\text { 1. Proportion of } \\
\text { patients receiving } \\
\text { stratified number of } \\
\text { PT visits }\end{array}$ & $\begin{array}{l}\text { The number of visits to PT } \\
\text { within actual treatment } \\
\text { period for patients sub- } \\
\text { grouped in low- or medium- } \\
\text { /high-risk of persistent } \\
\text { symptoms }\end{array}$ & $\begin{array}{l}\text { Fewer visits in the SBT low- } \\
\text { risk subgroup (1-3 visits) and } \\
\text { more in the SBT medium- } \\
\text { /high-risk subgroup ( } \geq 4 \\
\text { visits), Register data }\end{array}$ \\
\hline & $\begin{array}{l}\text { 2. Proportion of } \\
\text { patients receiving } \\
\text { educational } \\
\text { interventions }\end{array}$ & $\begin{array}{l}\text { Reassuring } \\
\text { information/education on } \\
\text { pain, pain management and } \\
\text { relapse prevention applying } \\
\text { behavioural medicine } \\
\text { approaches }\end{array}$ & $\begin{array}{l}\text { Increase overall use and } \\
\text { stratified use with higher } \\
\text { proportions in higher risk } \\
\text { groups, CRPE }\end{array}$ \\
\hline & $\begin{array}{l}\text { 3. Proportion of } \\
\text { patients receiving } \\
\text { exercise interventions }\end{array}$ & $\begin{array}{l}\text { Supervised/monitored } \\
\text { individualised home or } \\
\text { group-based exercise } \\
\text { programme }\end{array}$ & Increase overall use, CRPE \\
\hline & $\begin{array}{l}\text { 4. Proportion of } \\
\text { patients receiving } \\
\text { manual therapy }\end{array}$ & $\begin{array}{l}\text { Joint and/or soft-tissue } \\
\text { mobilisation/manipulation }\end{array}$ & $\begin{array}{l}\text { Short-term use as a part of } \\
\text { tmultimodal treatment } \\
\text { when LBP with segmental } \\
\text { movement impairments is } \\
\text { found, CRPE }\end{array}$ \\
\hline & $\begin{array}{l}\text { 5. Proportion of } \\
\text { patients receiving } \\
\text { acupuncture }\end{array}$ & Acupuncture & $\begin{array}{l}\text { Short-term use as an adjunct } \\
\text { to treatment intervention } \\
2 \& 3 \text { when additional short- } \\
\text { term analgesic effect as } \\
\text { goal, CRPE }\end{array}$ \\
\hline & $\begin{array}{l}\text { 6. Proportion of } \\
\text { patients receiving } \\
\text { non-evidence-based } \\
\text { treatments }\end{array}$ & $\begin{array}{l}\text { Passive physical modalities } \\
\text { e.g. corset, static traction, } \\
\text { electrotherapy, ultrasound, } \\
\text { *non-evidence-based } \\
\text { manual therapy, }{ }^{* *} \text { non- } \\
\text { evidence-based } \\
\text { acupuncture }\end{array}$ & Decrease use, $C R P E$ \\
\hline
\end{tabular}

CPG = Clinical Practice Guideline, PT = physiotherapy, LBP = Low Back pain, MRI = Magnetic Resonance Imaging, CT = Computerised Tomography, CRPE = clinical reasoning and process evaluation data from physiotherapists, SBT = STarT Back Screening Tool, + multimodal treatment $=$ exercise and/or educational interventions, *non-evidence manual therapy = manual therapy without reported segmental movement impairments or used as unimodal intervention not combined with exercise and/or educational interventions, ${ }^{* *}$ non-evidence acupuncture $=$ acupuncture used as unimodal intervention not combined with exercise and educational interventions 
Table 9. Clinical practice quality index for PTs' care delivery to patients with LBP used in Papers III-IV

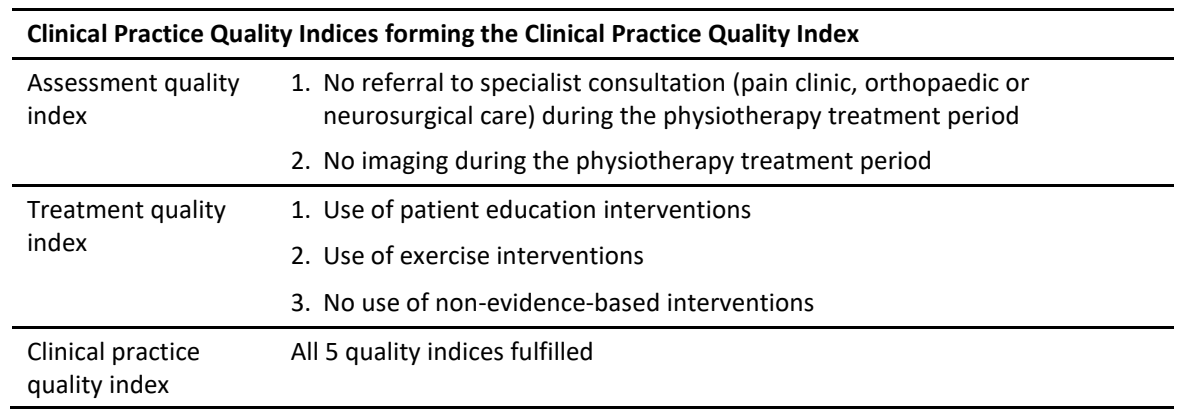

\section{Patient-reported outcomes (Study B, Paper IV)}

This was a stepped, single-blinded cluster RCT. To evaluate effectiveness outcomes, patients with LBP were allocated to the BetterBack $;$ MoC or routine care. Patient-reported outcomes were evaluated. An additional explorative analysis of patient outcomes was performed, with patients grouped according to fulfilment of a clinical practice quality index (CPQI) or non-fulfilment. Descriptive statistics were used to compare independent variables at baseline between PTs and patients in intervention and control groups using unpaired Student's t-test or Mann-Whitney U test for continuous data and the Chi-Square test for categorical data. Based on the a-priori hypothesised small effect size of $\mathrm{d}=$ 0.35 on changes in primary and secondary outcomes with a one tailed $\mathrm{p}=$ 0.05 for the superiority of the intervention compared with control group and with 80\% power, 204 participants were needed. Adjusting for the design effect due to cluster randomisation, an ICC of 0.01 , a cluster autocorrelation of 0.80 and the dog-leg stepped design, 100 participants in each cluster step with a total of 402 participants over 2.4 clusters were needed for $80 \%$ statistical power (129). For our primary outcome time period baseline to three-month follow-up for ODI and NRS-LBP, a significance level of $\mathrm{p} \leq 0.05$ was used in line with the a-priori protocol, but for all other measures and time points a Bonferroni corrected $\mathrm{p} \leq 0.017$ was used to adjust for multiplicity of repeated measures.

The study-cohort-specific minimal clinically important difference (MCID) was assessed for primary and secondary outcomes at 3-, 6- and 12month follow-up to interpret the between and within group changes. MCID was based on recommendations for use of an anchor method where PGIC could serve as a sufficiently strong anchor when correlation coefficients are $\geq 0.30$ in association to PROMs (130). The OCP on a Receiver Operating Curve (ROC) was defined by the sensitivity and specificity with lowest percentage of misclassification regarding improvement on the PGIC (131). 
PGIC was dichotomised into not improved $=-5$ to 0 and improved $=1$ to 5 . As a secondary criterion, if the OCP indicated worsening of the PROM, the nearest value indicating an improvement on the PROM was chosen as the MCID. An Area Under the Curve (AUC) greater than or equal to 0.70 represents satisfactory accuracy for the model $(132,133)$.

PROMs data at different time points were analysed and compared according to an intention to treat principle using restricted maximum likelihood approach in mixed models adjusted for unstructured covariance structure (134). A sensitivity analysis comparing intention to treat data with per protocol data showed no significant differences. However, analyses of the PEI and PGIC, which are transition rating scales with no baseline values, were therefore analysed only on per protocol data. Generalised mixed models were used for dichotomous dependent variables and linear mixed model were used for continuous dependent variables. All outcome scores were corrected for the clustering effects. ICC was calculated according to the three regions that formed the control and intervention groups, with equal groups assumed (129). In the secondary analysis the CPQI was used as the dependent variable. For PTs'care to be considered as fulfilling the CPQI, the care to patients had to have all five clinical practice indices fulfilled. This group is defined as the CPQI-adherent care group (Table 9) while the fulfilment of less than five clinical practice indices was defined as non CPQI adherent group. Between-group and within-group changes from baseline across all follow-up assessments were analysed for all outcome measures with a Bonferroni adjusted two-sided significance level of $\mathrm{p} \leq$ 0.017. IBM SPSS version 25 with the statistical package R 3.6.0 ( $\mathrm{R}$ Core Team, 2018) was used in all statistical analyses.

\section{Ethical considerations}

The studies were performed in accordance with the Declaration of Helsinki ethical principles for medical research. All patients and healthcare managers provided written informed consent to participate prior to inclusion. The study was described in the written information document to participating patients, and it was clearly stated that participation is voluntary and that non-participation in the studies would not affect future treatment. Furthermore, one could choose to withdraw one's participation at any time without explanation. All data and information would be anonymized. The Regional Ethics Committee in Linköping considered that a written consent from each participating physiotherapist was not necessary because the intervention was at the practice level, and the participating physiotherapists consented to participate. There was no position of dependence between researchers and study participants, or commercial interest tied to these studies. The results of the studies are not 
presented such individual study participants can be identified. The studies were approved by the Swedish Ethical Review Authority in Linköping.

All data were anonymised and stored in a fire-safe security locker at Linköping University, in order to fulfil data protection requirements for storage of personal information. To maintain anonymity, only coded data was discussed by the involved researchers. 


\section{RESULTS}

\section{The Determinants of Implementation Behaviour Questionnaire (DIBQ) (Papers I and II)}

\section{Questionnaire development (Study A)}

The translated Swedish and Danish versions of the original DIBQ are presented as Appendix S1 and Appendix S2 in study A (135). The tailoring of the original 93-item DIBQ resulted in a 28 -item version to assess clinicians' expectations to implementation. (Table 10). Of these 28 items, 18 were included based upon selection by project leaders + having $\geq 80 \%$ CVI from Danish and Swedish experts. A further 10 items were selected by project leaders and had $\geq 80 \%$ CVI either by the Danish or the Swedish experts. This tailored 28-item DIBQ-t had a representation of 10 of the initial 18 DIBQ domains and covered all the categories of the COM-B model for Capability (domains: Knowledge, Skills and Behaviour regulation), Opportunity (domains: Organisation, Social influence, Patient and Innovation) and Motivation (domains: Beliefs about capabilities, Beliefs about consequences and Intentions). DIBQ-t with 28 items demonstrated good feasibility with only $2 \%$ missing data from 598 Danish and Swedish clinicians. At the domain level, at least $72 \%$ of the clinicians rated positive expectations to implementation except for the domain Behaviour regulation where $55 \%$ strongly agreed/agreed with the items in the domain (Figure 9). When interpretating the results with linkage to the COM-B, the strongest domains were Knowledge and Skills within the COM-B category Capability where $\geq 90 \%$ strongly agreed/agreed with the items. However, in this Capability category the domain Behaviour regulation had the lowest rating, with $44 \%$ having neutral or negative expectation of implementation. This especially regarding the item number 28 "planning management of unmotivated patients" where $63 \%$ clinicians rated neutral /negative expectations to implementation. In three domains of the COM-B category Opportunity, 26-28\% of clinicians responded with neutral or negative expectations to implementation. 
Table 10. DIBQ-t 28 item: Expectations for implementation: domains and items

\begin{tabular}{|c|c|c|}
\hline DIBQ-t item & TDF domain & Items \\
\hline DIBQ-t 1 & knowledge & I know how to deliver Better Back/GLA:D Back following the programme. \\
\hline DIBQ-t 2 & knowledge & Objectives of Better Back/GLA:D Back and my role in this are clearly defined for me. \\
\hline DIBQ-t 3 & skills & I have the skills to deliver Better Back/GLA:D Back. \\
\hline DIBQ-t 4* & $\begin{array}{l}\text { beliefs about } \\
\text { capability }\end{array}$ & I am confident that I can deliver Better Back/GLA:D Back. \\
\hline DIBQ-t 5 & $\begin{array}{l}\text { beliefs about } \\
\text { capability }\end{array}$ & I expect that delivering Better Back/GLA:D Back is (very easy - very difficult). \\
\hline DIBQ-t 6 & $\begin{array}{l}\text { beliefs about } \\
\text { capability }\end{array}$ & I expect that performing the intake is (very easy - very difficult). \\
\hline DIBQ-t 7 & $\begin{array}{l}\text { beliefs about } \\
\text { capability }\end{array}$ & I expect that delivering the training programme is (very easy - very difficult). \\
\hline DIBQ-t 8 & $\begin{array}{l}\text { beliefs about } \\
\text { capability }\end{array}$ & $\begin{array}{l}\text { I expect that giving attention to participant's maintenance of physical activity } \\
\text { behaviour outside Better Back/GLA:D Back is (very easy - very difficult). }\end{array}$ \\
\hline DIBQ-t 9 & $\begin{array}{l}\text { beliefs about } \\
\text { capability }\end{array}$ & $\begin{array}{l}\text { I expect that reporting about the Better Back/GLA:D Back to the referring } \\
\text { professional is (very easy - very difficult). }\end{array}$ \\
\hline DIBQ-t 10 & $\begin{array}{l}\text { beliefs about } \\
\text { consequences }\end{array}$ & $\begin{array}{l}\text { I expect that delivering Better Back/GLA:D Back is (not worthwhile at all - very } \\
\text { worthwhile). }\end{array}$ \\
\hline DIBQ-t 11 & $\begin{array}{l}\text { beliefs about } \\
\text { consequences }\end{array}$ & If I deliver Better Back/GLA:D Back, Better Back/GLA:D Back will be most effective. \\
\hline DIBQ-t 12 & $\begin{array}{l}\text { beliefs about } \\
\text { consequences }\end{array}$ & $\begin{array}{l}\text { If I deliver Better Back/GLA:D Back, it will help participants to be able to cope better } \\
\text { with their back problems. }\end{array}$ \\
\hline DIBQ-t 13* & $\begin{array}{l}\text { beliefs about } \\
\text { consequences }\end{array}$ & $\begin{array}{l}\text { I expect that, when I deliver Better Back/GLA:D Back, I get recognition from the } \\
\text { work context. }\end{array}$ \\
\hline DIBQ-t 14 & intentions & I intend to deliver Better Back/GLA:D Back in the next three months. \\
\hline DIBQ-t 15 & innovation & It will be possible to tailor Better Back/GLA:D Back to participants' needs. \\
\hline DIBQ-t 16 & innovation & It will be possible to tailor Better Back/GLA:D Back to professionals' needs. \\
\hline DIBQ-t 17 & innovation & Better Back/GLA:D Back will be compatible with daily practice. \\
\hline DIBQ-t 18 & innovation & Better Back/GLA:D Back will be simple to deliver. \\
\hline DIBQ-t 19 & organisation & $\begin{array}{l}\text { I expect that, in the organisation I work in, all necessary resources are available to } \\
\text { deliver Better Back/GLA:D Back. }\end{array}$ \\
\hline DIBQ-t 20 & organisation & $\begin{array}{l}\text { I expect that I can count on support from the management of the organisation I } \\
\text { work in when things get tough in the programme. }\end{array}$ \\
\hline DIBQ-t 21 & patient & I expect that participants of Better Back/GLA:D Back are motivated. \\
\hline DIBQ-t 22 & patient & $\begin{array}{l}\text { I expect that participants of Better Back/GLA:D Back are positive about Better } \\
\text { Back/GLA:D Back. }\end{array}$ \\
\hline DIBQ-t 23 & social influences & $\begin{array}{l}\text { Most people who are important to me think that I should deliver Better Back/GLA:D } \\
\text { Back. }\end{array}$ \\
\hline DIBQ-t 24 & social influences & $\begin{array}{l}\text { Professionals with whom I deliver Better Back/GLA:D Back think I should deliver } \\
\text { Better Back/GLA:D Back. }\end{array}$ \\
\hline DIBQ-t 25 & social influences & $\begin{array}{l}\text { I can count on support from professionals with whom I deliver Better Back/GLA:D } \\
\text { Back when things get tough with delivering Better Back/GLA:D Back. }\end{array}$ \\
\hline DIBQ-t 26 & $\begin{array}{l}\text { behavioural } \\
\text { regulation }\end{array}$ & I have a clear plan of how I will deliver Better Back/GLA:D Back. \\
\hline DIBQ-t 27 & $\begin{array}{l}\text { behavioural } \\
\text { regulation }\end{array}$ & I have a clear plan when I will deliver Better Back/GLA:D Back. \\
\hline DIBQ-t 28 & $\begin{array}{l}\text { behavioural } \\
\text { regulation }\end{array}$ & $\begin{array}{l}\text { I have a clear plan about delivering Better Back/GLA:D Back when participants are } \\
\text { not motivated. }\end{array}$ \\
\hline
\end{tabular}

*Item not included in the final 26-item DIBQ-t 


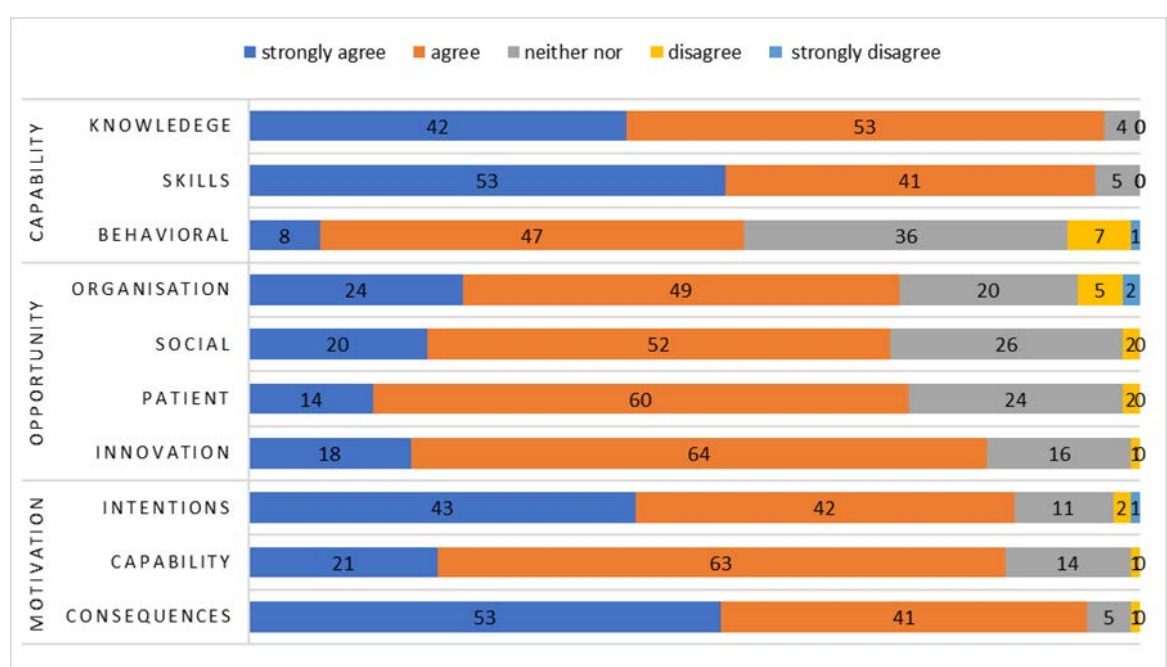

Figure 9. Distribution of responses of the DIBQ-t at domain level in percentage on a $5^{-}$ point Likert scale, sorted by COM-B

The confirmatory factor analysis (CFA) showed good fit after removing two items with the lowest domain loadings (Table 11). These items were item 4 "I am confident that I can deliver BetterBack-;/GLA:D Back" and item 13 "I expect that, when I deliver BetterBack-(-)GLA:D Back, I get recognition from the work context". The estimated factor loadings of the items related to the domains were between 0.365 and 0.819 where 3 items (items 12,15 and 16 were below 0.4 , however with a p-value $<0.001$. After removing these two weakest loading items (items 4 and 13), the different domains in the questionnaire did not overlap and with a Cronbach's Alpha value of 0.90 where over 0.70 indicates an acceptable internal consistency (136) (Table 12). This 26-item questionnaire was the DIBQ version used in Paper II.

Table 11. Results from the construct validity testing after removal of 2 items

\begin{tabular}{ccccccc}
\hline $\mathbf{X}^{2}$ & Df & p-value & CFI & TLI & RMSEA (90\% CI) & SRMR \\
\hline 635.844 & 256 & $<0.001$ & 0.933 & 0.916 & $0.050(0.045-0.055)$ & 0.047 \\
\hline
\end{tabular}

CFI = Comparative Fit Index, TLI = Tucker-Lewis Index, RMSEA = Root Mean Square Error of

Approximation, $\mathrm{SRMR}=$ Standardised Root Mean Square Residual 
Table 12. Estimated factor loading ranges and internal consistency for the domains

\begin{tabular}{lccc}
\hline DIBQ Domain & $\begin{array}{c}\text { Items } \\
(\mathrm{n})\end{array}$ & $\begin{array}{c}\text { Estimated factor loading } \\
\text { range for items }\end{array}$ & $\begin{array}{c}\text { Reliability analysis -Internal } \\
\text { consistency (Cronbach's alpha) }\end{array}$ \\
\hline Knowledge & 2 & $0.455-0.490$ & 0.788 \\
Skills & 1 & 0.632 & 1.000 \\
Beliefs about capabilities & 5 & $0.406-0.589$ & 0.779 \\
Beliefs about consequences & 3 & $0.399-0.443$ & 0.730 \\
Intentions & 1 & 0.819 & 1.000 \\
Innovation & 4 & $0.365-0.536$ & 0.717 \\
Organisation & 2 & $0.689-0.696$ & 0.721 \\
Patient & 2 & $0.521-0.582$ & 0.855 \\
Social influences & 3 & $0.435-0.652$ & 0.736 \\
Behavioural regulation & 3 & $0.435-0.739$ & 0.774 \\
\hline
\end{tabular}

\section{Longitudinally evaluation with DIBQ (Paper II)}

Figure 10 presents the CONSORT flowchart describing the result of recruitment and data collection in Paper II. The DIBQ version used in longitudinal evaluation was the 26-item version with the best fit according to the CFA in Study A (DIBQ-t). The RMANOVA displayed high implementation behaviours (scores $>50$ ) in all DIBQ domains over time, implying that all were potential facilitators of implementation (Tables 13 ac and Figure 11). Directly after the two-day workshop, expected determinants of implementation behaviours were moderate to high potential facilitators (scores $>75$ ) for six of the 10 domains: Knowledge, Skills, Social influence, Innovation, Beliefs about consequences and Intention domains. The four domains: Behaviour regulation, Organisation, Patient and Beliefs about capabilities were small to moderate potential facilitators (scores 50-75). Pairwise comparisons between directly after workshop and 3-month follow-up showed a significant decreased in all domains except for Organisation, Social influence and Patients. However, the domain Organisation and Social influence decreased at 12-month follow-up while Knowledge, Skills and Beliefs about capabilities returned to initial levels. Ratings of patients' expectation of the MoC were maintained at $3^{-}$and 12- month follow-up. With the linkage to COM-B category Capability, the DIBQ domains showed a pattern of short-term decrease followed by a long-term sustainability. For COM-B Opportunity, PTs maintained the view that patients have positive expectations (DIBQ domain Patients) for the MoC. However, both the organisation and social influence domains showed a pattern of short-termed sustainability, but they decreased in the long term. Furthermore, the domain innovation 
decreased both in short and long term. Similarly, with regards to COM-B Motivation, a decrease in all domains was shown in both the short and long term, except for beliefs about capabilities that returned to initial levels at 12 months.

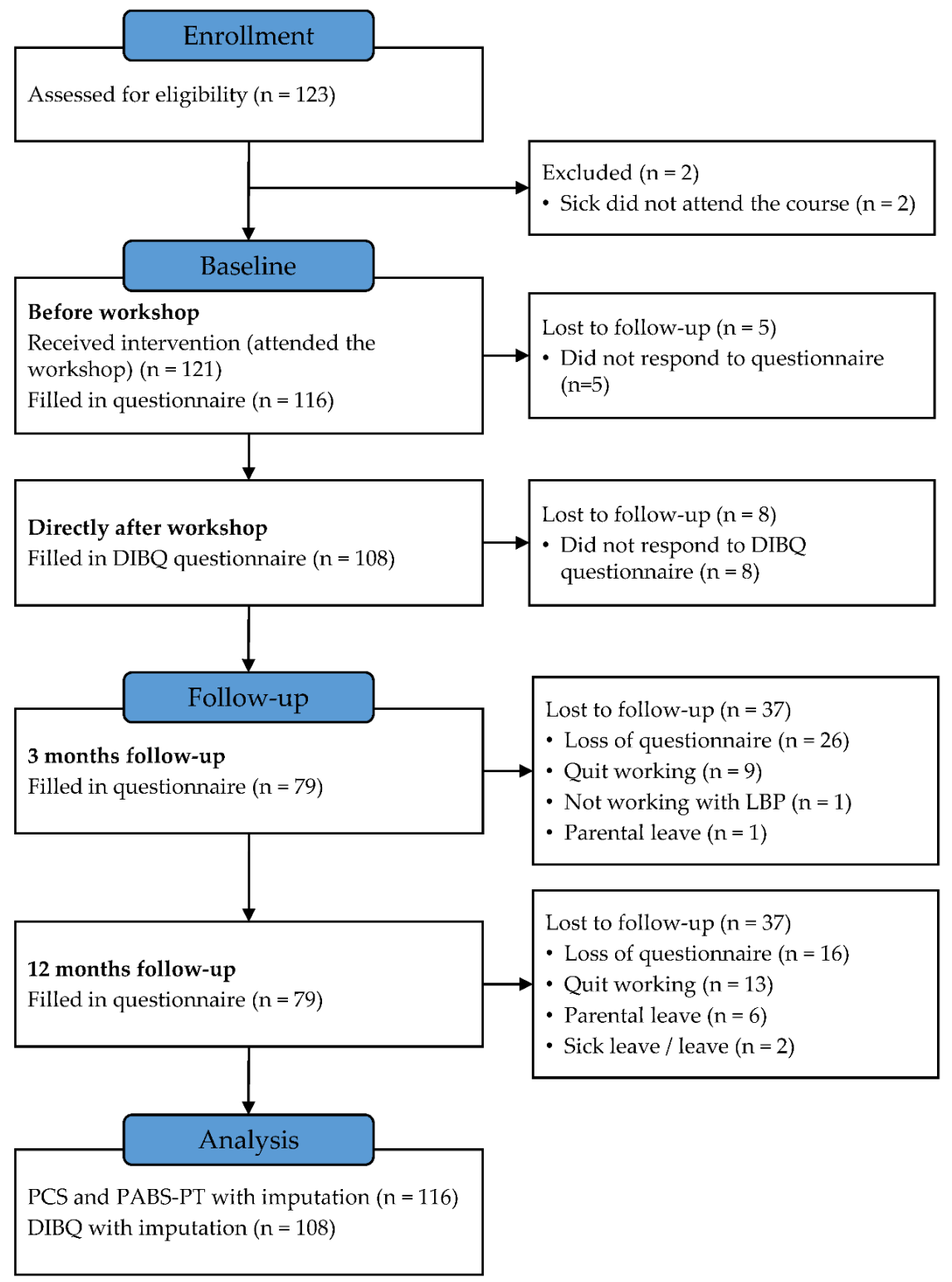

Figure 10. CONSORT flowchart of participants in Paper II 
Table 13a. Changes in physiotherapists' determinants of implementation behaviour from directly after workshop, COM-B Capability $(n=108)$

\begin{tabular}{|c|c|c|c|c|}
\hline \multirow[b]{2}{*}{$\begin{array}{l}\text { COM-B } \\
\text { Capability }\end{array}$} & \multirow[b]{2}{*}{ Mean (SD) } & \multirow{2}{*}{$\begin{array}{c}\text { Within-subjects effects } \\
\text { F-value; } p \text {-value; } \\
\text { Effect Size }\end{array}$} & \multicolumn{2}{|c|}{ Within-subjects simple contrasts } \\
\hline & & & $\begin{array}{l}\text { Change from } \\
\text { baseline } \\
\text { Mean }(95 \% \mathrm{Cl})\end{array}$ & $\begin{array}{l}\text { F-value; p-value; } \\
\text { Effect Size }\end{array}$ \\
\hline Knowledge & & $\begin{array}{c}F(2,214)=12.6 ; p<0.001 \\
\eta_{p}^{2}=0.105\end{array}$ & & \\
\hline Directly after & $82.3(14.4)$ & & & \\
\hline 3 months after & $74.9(17.1)$ & & $-7.4(-10.8$ to -4.1$)$ & $\begin{array}{c}F(1,107)=19.1 ; p<0.001 ; \\
\eta_{p}^{2}=0.152\end{array}$ \\
\hline $\begin{array}{l}12 \text { months } \\
\text { after }\end{array}$ & $80.7(16.8)$ & & $-1.6(-4.7$ to 1.5$)$ & $\begin{array}{c}F(1,107)=1.1 ; p=0.30 \\
\eta_{p}^{2}=0.010\end{array}$ \\
\hline Skills & & $\begin{aligned} \mathrm{F}(1.7,182) & =2.3 ; \mathrm{p}=0.11 \\
\eta_{\mathrm{p}}^{2} & =0.021\end{aligned}$ & & \\
\hline Directly after & $85.6(18.2)$ & & & \\
\hline 3 months after & $81.5(19.4)$ & & $-4.2(-8.3$ to -0.1$)$ & $\begin{array}{c}F(1,107)=4.1 ; p<0.05 \\
\eta_{p}^{2}=0.037\end{array}$ \\
\hline $\begin{array}{l}12 \text { months } \\
\text { after }\end{array}$ & $84.3(19.5)$ & & $-1.4(-5.9$ to 3.1$)$ & $\begin{array}{c}F(1,107)=0.4 ; p=0.54 \\
\eta_{p}^{2}=0.004\end{array}$ \\
\hline $\begin{array}{l}\text { Behavioural } \\
\text { regulation }\end{array}$ & & $\begin{aligned} F(2,214) & =2.1 ; p=0.13 \\
\eta_{p}^{2} & =0.019\end{aligned}$ & & \\
\hline Directly after & $61.4(18.5)$ & & & \\
\hline 3 months after & $58.5(17.6)$ & & $-2.9(-6.4$ to 0.5$)$ & $\begin{array}{c}F(1,107)=2.9 ; p<0.05 \\
\eta_{p}^{2}=0.026\end{array}$ \\
\hline $\begin{array}{l}12 \text { months } \\
\text { after }\end{array}$ & $58.5(17.0)$ & & $-2.9(-6.4$ to 0.6$)$ & $\begin{array}{c}F(1,107)=2.7 ; p<0.05 \\
\eta_{p}^{2}=0.025\end{array}$ \\
\hline
\end{tabular}


Table 13b. Changes in physiotherapists' determinants of implementation behaviour from directly after workshop, COM-B Opportunity $(n=108)$

\begin{tabular}{|c|c|c|c|c|}
\hline \multirow[b]{2}{*}{$\begin{array}{l}\text { COM-B } \\
\text { Opportunity }\end{array}$} & \multirow[b]{2}{*}{ Mean (SD) } & \multirow{2}{*}{$\begin{array}{l}\text { Within-subjects effects } \\
\text { F-value; } p \text {-value; } \\
\text { Effect Size }\end{array}$} & \multicolumn{2}{|c|}{ Within-subjects simple contrasts } \\
\hline & & & $\begin{array}{l}\text { Change from } \\
\text { baseline } \\
\text { Mean }(95 \% \mathrm{Cl})\end{array}$ & $\begin{array}{l}\text { F-value; p-value; } \\
\text { Effect Size }\end{array}$ \\
\hline Organisation & & $\begin{aligned} F(1.9,201) & =5.1 ; p<0.01 \\
\eta_{p}^{2} & =0.046\end{aligned}$ & & \\
\hline Directly after & $72.9(23.0)$ & & & \\
\hline 3 months after & $68.9(20.1)$ & & $-4.1(-8.2$ to 0.1$)$ & $\begin{array}{c}F(1,107)=3.8 ; p=0.05 \\
\eta_{p}^{2}=0.034\end{array}$ \\
\hline $\begin{array}{l}12 \text { months } \\
\text { after }\end{array}$ & $66.2(16.1)$ & & $-6.7(-11.4$ to 2.0$)$ & $\begin{array}{c}F(1,107)=8.1 ; p<0.01 \\
\eta_{p}^{2}=0.070\end{array}$ \\
\hline Social influence & & $\begin{array}{c}F(2,214)=8.6 ; p<0.001 ; \\
\eta_{p}^{2}=0.074\end{array}$ & & \\
\hline Directly after & 76.9 (17.9) & & & \\
\hline 3 months after & $73.0(20.4)$ & & $-3.9(-8.0$ to 0.3$)$ & $\begin{array}{c}F(1,107)=3.4 ; p=0.07 \\
\eta_{p}^{2}=0.031\end{array}$ \\
\hline $\begin{array}{l}12 \text { months } \\
\text { after }\end{array}$ & $68.5(16.2)$ & & $-8.3(-12.3$ to -4.3$)$ & $\begin{array}{c}F(1,107)=17.1 ; p<0.001 ; \\
\eta_{p}^{2}=0.138\end{array}$ \\
\hline Patients & & $\begin{aligned} F(2,214) & =0.3 ; p=0.77 \\
\eta_{p}^{2} & =0.002\end{aligned}$ & & \\
\hline Directly after & $62.3(14.1)$ & & & \\
\hline 3 months after & $61.1(14.7)$ & & $-1.2(-4.7$ to 2.3$)$ & $\begin{array}{c}F(1,107)=0.4 ; p=0.51 \\
\eta_{p}^{2}=0.004\end{array}$ \\
\hline $\begin{array}{l}12 \text { months } \\
\text { after }\end{array}$ & $62.0(13.3)$ & & $-0.2(-3.7$ to 3.2$)$ & $\begin{array}{c}F(1,107)=0.2 ; p=0.90 \\
\eta_{p}^{2}<0.001\end{array}$ \\
\hline Innovation & & $\begin{array}{c}F(1.9,203)=23.4 ; p<0.001 \\
\eta_{p}^{2}=0.180\end{array}$ & & \\
\hline Directly after & $77.8(14.5)$ & & & \\
\hline 3 months after & $70.0(16.5)$ & & $-7.8(-10.9$ to -4.8$)$ & $\begin{array}{c}F(1,107)=25.7 ; p<0.001 ; \\
n_{p}^{2}=0.194\end{array}$ \\
\hline $\begin{array}{l}12 \text { months } \\
\text { after }\end{array}$ & $68.4(18.3)$ & & $-9.4(-12.6$ to -6.3$)$ & $\begin{array}{c}F(1,107)=35.9 ; p<0.001 ; \\
\eta_{p}^{2}=0.251\end{array}$ \\
\hline
\end{tabular}


Table 13c. Changes in physiotherapists' determinants of implementation behaviour from directly after workshop, COM-B Motivation $(n=108)$

\begin{tabular}{|c|c|c|c|c|}
\hline \multirow[b]{2}{*}{$\begin{array}{l}\text { COM-B } \\
\text { Motivation }\end{array}$} & \multirow[b]{2}{*}{ Mean (SD) } & \multirow{2}{*}{$\begin{array}{c}\text { Within-subjects effects } \\
\text { F-value; p-value; } \\
\text { Effect Size }\end{array}$} & \multicolumn{2}{|c|}{ Within-subjects simple contrasts } \\
\hline & & & $\begin{array}{l}\text { Change from } \\
\text { baseline } \\
\text { Mean }(95 \% \mathrm{Cl})\end{array}$ & $\begin{array}{l}\text { F-value; p-value; } \\
\text { Effect Size }\end{array}$ \\
\hline $\begin{array}{l}\text { Beliefs about } \\
\text { Capabilities }\end{array}$ & & $\begin{array}{c}F(2,214)=2.9 ; p=0.06 ; \\
\eta_{p}^{2}=0.026\end{array}$ & & \\
\hline Directly after & $71.6(16.5)$ & & & \\
\hline 3 months after & $66.8(19.7)$ & & $-4.2(-8.3$ to -0.1$)$ & $\begin{array}{c}F(1,107)=5.7 ; p<0.05 \\
\eta_{p}^{2}=0.051\end{array}$ \\
\hline $\begin{array}{l}12 \text { months } \\
\text { after }\end{array}$ & $69.5(17.2)$ & & $-2.1(-6.4$ to 2.2$)$ & $\begin{array}{c}F(1,107)=0.9 ; p=0.34 \\
\eta_{p}^{2}=0.009\end{array}$ \\
\hline $\begin{array}{l}\text { Beliefs about } \\
\text { consequences }\end{array}$ & & $\begin{array}{c}F(2,214)=41.2 ; p<0.001 \\
\eta_{p}^{2}=0.278\end{array}$ & & \\
\hline Directly after & $79.7(15.0)$ & & & \\
\hline 3 months after & $68.4(19.4)$ & & $-11.3(-14.3$ to -8.4$)$ & $\begin{array}{c}F(1,107)=57.4 ; p<0.001 ; \\
\eta_{p}^{2}=0.349\end{array}$ \\
\hline $\begin{array}{l}12 \text { months } \\
\text { after }\end{array}$ & $67.1(18.7)$ & & $-12.7(-15.7$ to $-9,6)$ & $\begin{array}{c}F(1,107)=65.8 ; p<0.001 ; \\
\eta_{p}^{2}=0.381\end{array}$ \\
\hline Intentions & & $\begin{array}{c}F(1.8,196)=38.6 ; p<0.001 \\
\eta_{p}^{2}=0.265\end{array}$ & & \\
\hline Directly after & $85.4(21,7)$ & & & \\
\hline 3 months after & $79.6(23.5)$ & & $-5.8(-10.0$ to -1.6$)$ & $\begin{array}{c}F(1,107)=7.4 ; p<0.01 \\
\eta_{p}^{2}=0.065\end{array}$ \\
\hline $\begin{array}{l}12 \text { months } \\
\text { after }\end{array}$ & $64.6(25.6)$ & & $\begin{array}{c}-20.8(-26.3 \text { to }- \\
15.3)\end{array}$ & $\begin{array}{c}F(1,107)=56.9 ; p<0.001 ; \\
\eta_{p}^{2}=0.347\end{array}$ \\
\hline
\end{tabular}




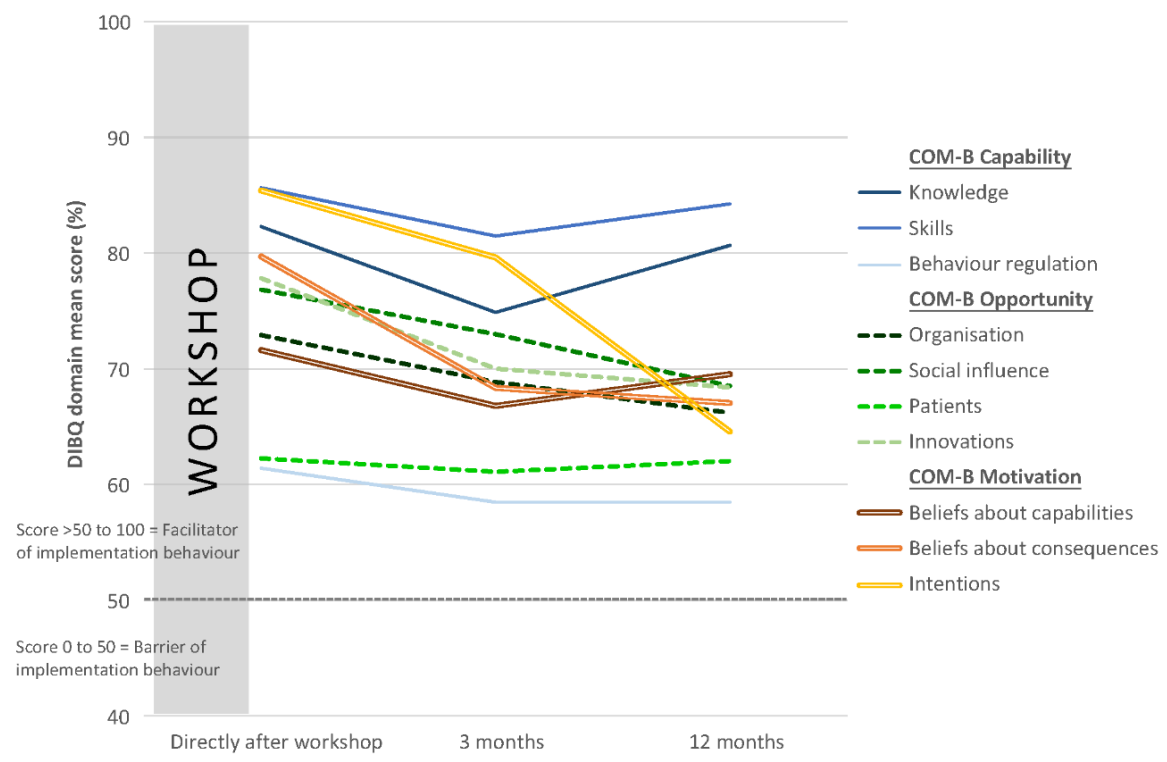

Figure 11. Changes in physiotherapists' determinants of implementation behaviour from directly after workshop $(\mathrm{n}=108)$ COM-B $=$ Capability Opportunity Motivation and Behaviour, DIBQ = Determinants of Implementation Behaviour

\section{Confidence, attitudes and beliefs (Paper II)}

The RMANOVA from 116 PTs displayed significant improvement in the primary outcome, (PCS), over time, with large effect size $\left(\eta_{p^{2}}=0.197, p<\right.$ o.001). This improvement of confidence in diagnosing and managing LBP was evident in all follow-ups. Changes in attitudes and beliefs measured with PABS-PT was only significant at 12-month follow-up with a higher biopsychosocial orientation, $\left(\eta_{\mathrm{p}}^{2}=0.071, \mathrm{p}<0.01\right)$ and lower biomedical orientation, $\left(\eta_{p^{2}}=0.136, p<0.001\right)$ (Table 14). 
Table 14. Changes in physiotherapists' self-confidence, pain attitudes and beliefs from before to after the workshop $(n=116)$

\begin{tabular}{|c|c|c|c|c|}
\hline & \multirow[b]{2}{*}{ Mean (SD) } & \multirow{2}{*}{$\begin{array}{c}\text { Within-subjects effects } \\
\text { F-value; p-value; } \\
\text { Effect Size }\end{array}$} & \multicolumn{2}{|c|}{ Within-subjects simple contrasts } \\
\hline & & & $\begin{array}{c}\text { Change from } \\
\text { baseline } \\
\text { Mean }(95 \% \mathrm{Cl})\end{array}$ & $\begin{array}{l}\text { F-value; p-value; } \\
\text { Effect Size }\end{array}$ \\
\hline PCS & & $\begin{array}{c}F(2.7,312)=28.3 ; p<0.001 ; \\
\eta_{p}^{2}=0.197\end{array}$ & & \\
\hline Before & $10.4(2.4)$ & & & \\
\hline Directly after & $8.8(2.1)$ & & $-1.6(-1.9$ to -1.2$)$ & $\begin{array}{c}F(1,115)=60.3 ; p<0.001 ; \\
\eta_{p}^{2}=0.344\end{array}$ \\
\hline 3 months after & $8.9(2.2)$ & & $-1.5(-1.9$ to -1.0$)$ & $\begin{array}{c}F(1,115)=44.1 ; p<0.001 ; \\
\eta_{p}^{2}=0.277\end{array}$ \\
\hline 12 months after & $8.7(2.2)$ & & $-1.7(-2.0$ to -1.3$)$ & $\begin{array}{c}F(1,115)=95.6 ; p<0.001 \\
\eta_{p}^{2}=0.454\end{array}$ \\
\hline PABS-PT, BM & & $\begin{aligned} F(2,230) & =7.2 ; p<0.001 \\
\eta_{p}^{2} & =0.059\end{aligned}$ & & \\
\hline Before & $32.0(7.0)$ & & & \\
\hline 3 months after & $31.2(6.9)$ & & $-0.8(-1.8$ to 0.1$)$ & $\begin{array}{c}F(1,115)=2.9 ; p=0.09 \\
\eta_{p}^{2}=0.024\end{array}$ \\
\hline 12 months after & $30.3(6.5)$ & & $-1.7(-2.5$ to -0.9$)$ & $\begin{array}{c}F(1,115)=18.1 ; p<0.001 ; \\
\eta_{p}^{2}=0.136\end{array}$ \\
\hline PABS-PT, BPS & & $\begin{aligned} F(2,230) & =4.2 ; p=0.016 \\
\eta_{p}^{2} & =0.035\end{aligned}$ & & \\
\hline Before & $38.9(4.8)$ & & & \\
\hline 3 months after & $39.6(4.1)$ & & $0.7(-0.1$ to 1.5$)$ & $\begin{array}{c}F(1,115)=2.9 ; p=0.09 \\
\eta_{p}^{2}=0.025\end{array}$ \\
\hline 12 months after & $40.0(3.7)$ & & 1.1 (0.4 to 1.8$)$ & $\begin{array}{c}F(1,115)=8.8 ; p<0.01 \\
\eta_{p}^{2}=0.071\end{array}$ \\
\hline
\end{tabular}

$\mathrm{SD}=$ Standard Deviation, $\mathrm{Cl}=$ Confidence Interval, $\eta_{\mathrm{p}}{ }^{2}=$ Partial Eta Squared, $\mathrm{PCS}=$ Practitioner Self-Confidence Scale (score 4-20, lower score indicates higher self-confidence), PABS-PT = Pain Attitudes and Beliefs Scale for Physiotherapists, $\mathrm{BM}=$ Biomedical orientation (score $10-60$ indicates higher score higher orientation), BPS = Biopsychosocial orientation (score 9 - 54, indicates higher score higher orientation)

\section{Adherence to clinical practice guidelines (Papers III and IV)}

A total of 500 patients were included, 278 were allocated to treatment by PTs who had received education in how to use the BetterBack MoC:; (intervention group), while 222 were allocated to routine physiotherapy care (control group) between April 2017 and March 2018. Figure 12 presents the CONSORT flow chart describing the result of recruitment and data collection. For variables: medical imaging, number of visits and referral to specialist consultation, complete registry data including all 500 patients were used. For PT-reported treatment, a smaller patient cohort with 388 patients was used because CRPE data was not as complete as register data. In this cohort there were 223 patients in the intervention 
group and 165 patients in the routine care group. Because the second cluster had a period of routine care before the implementation of BetterBack $-:$ MoC, 12 PTs treated patients during both routine care period and after the $\mathrm{MoC}$ implementation. Baseline characteristics between PTs and patients in the different groups did not differ significantly. However, the mean number of PT treatment sessions was higher in the intervention group with a mean of 4.6 (SD 3.8) sessions, compared to 3.1 (SD 2.7) sessions in the routine care group.

Regarding the primary outcome, the proportion of patients referred to specialist consultation was very low in both groups with no statistical difference (Table 15). However, less use of medical imaging produced a significant between-group difference in the assessment quality index, with 91.0\% adherence to the two guidelines in the intervention group versus $82.9 \%$ in the routine care group (OR 2.0;95\% CI 1.2 to $3.4 ; \mathrm{p}=0.01$ ). Furthermore, the register data showed a significantly higher stratification of number of PT visits based on SBT, with $54.7 \%$ adherence to this recommendation in intervention group versus $44.6 \%$ in routine care (OR $1.5 ; 95 \%$ CI 1.0 to $2.3 ; \mathrm{p}=0.04$ ).

The largest between-group differences were found in the proportions of patients receiving educational interventions. with $73.5 \%$ in the intervention versus $35.2 \%$ in routine care (OR 5.1; 95\% CI 3.3 to 8.0; $p<$ 0.001). When this analysis was sub-grouped according to SBT, the proportional odds were largest for the medium/high risk SBT group in the intervention group (OR 8.1; 95\% CI 4.4 to 14.9 ; $\mathrm{p}<0.001$ ).

The proportion of patients receiving exercise intervention was similar and high in both groups, with over $85 \%$ use in all patients. Passive treatments did not differ between groups; manual therapy was used in $11.2 \%$ of all LBP cases in the intervention versus $16.4 \%$ in routine care; and acupuncture was used in only four cases. The proportion of non-evidencebased treatment was significantly lower in the intervention group: $11.2 \%$ compared to $21.8 \%$ in routine care (OR $0.4 ; 95 \% \mathrm{CI} 0.2$ to $0.8 ; \mathrm{p}=0.015$ ).

The proportions of cases fulfilling the treatment quality index (use of exercise, educational interventions, and no use of non-evidence-based treatment) reported in Table 15 was significantly higher, with $66.4 \%$ fulfilment in the intervention group compared to $28.5 \%$ in routine care (OR $3.5 ; 95 \% \mathrm{CI} 1.7$ to $7.2 ; \mathrm{p}<0.001$ ). For the overall adherence (all five criteria from both the assessment and treatment quality index), a significantly higher proportion was found in the intervention group, with $58.7 \%$ fulfilment compared to $25.5 \%$ (OR 3.5 ; 95\% CI 1.9 to 6.6 ; $\mathrm{p}<0.001$ ). 


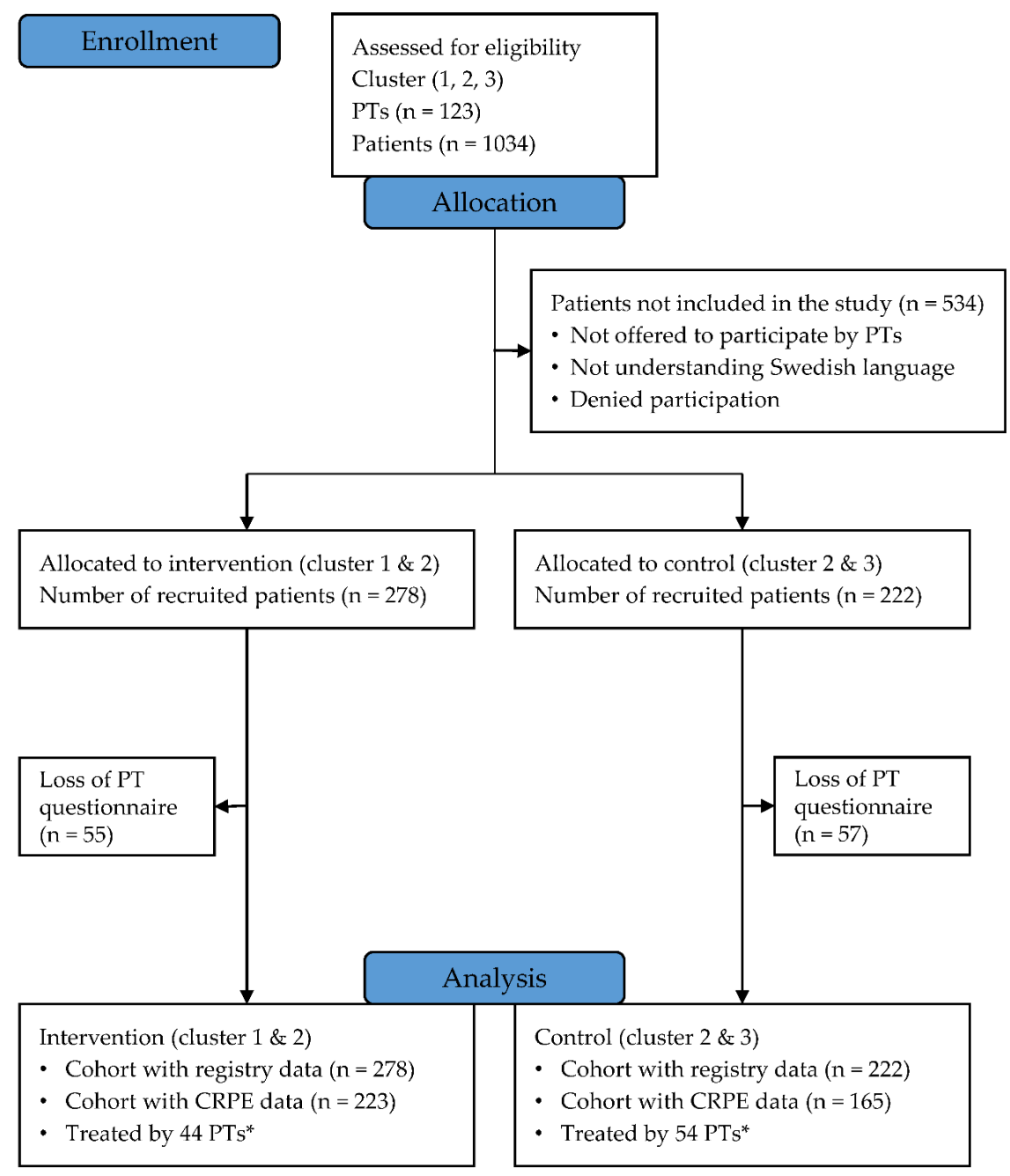

* 12 PTs in cluster 2 crossed over from control to intervention phase after implementation of the BetterBack@ MoC and therefore treated patients in both phases of the study

Figure 12. CONSORT flowchart of participants in Paper III. $P T=$ physiotherapists 


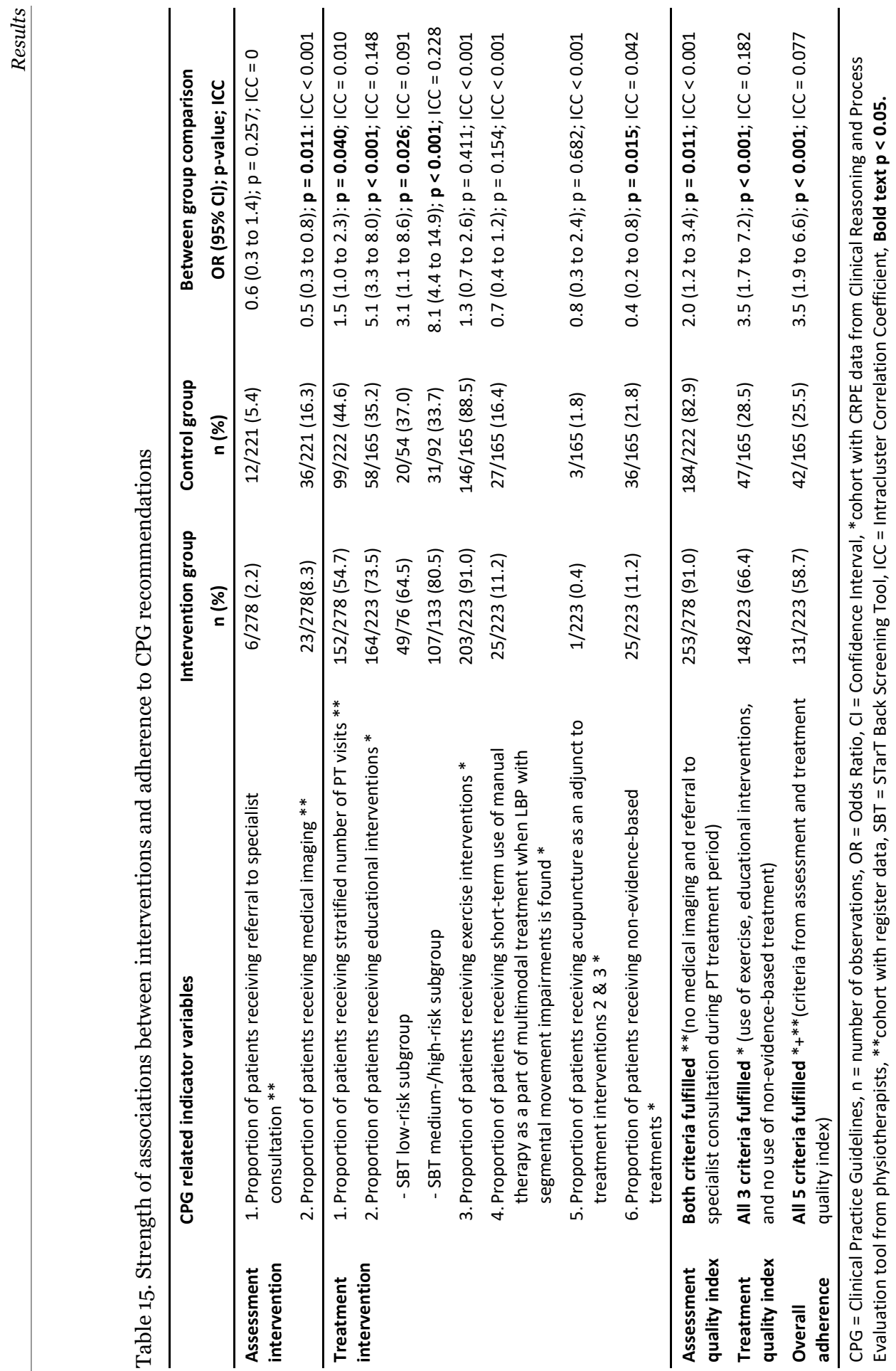




\section{Patient-reported outcomes (Paper IV)}

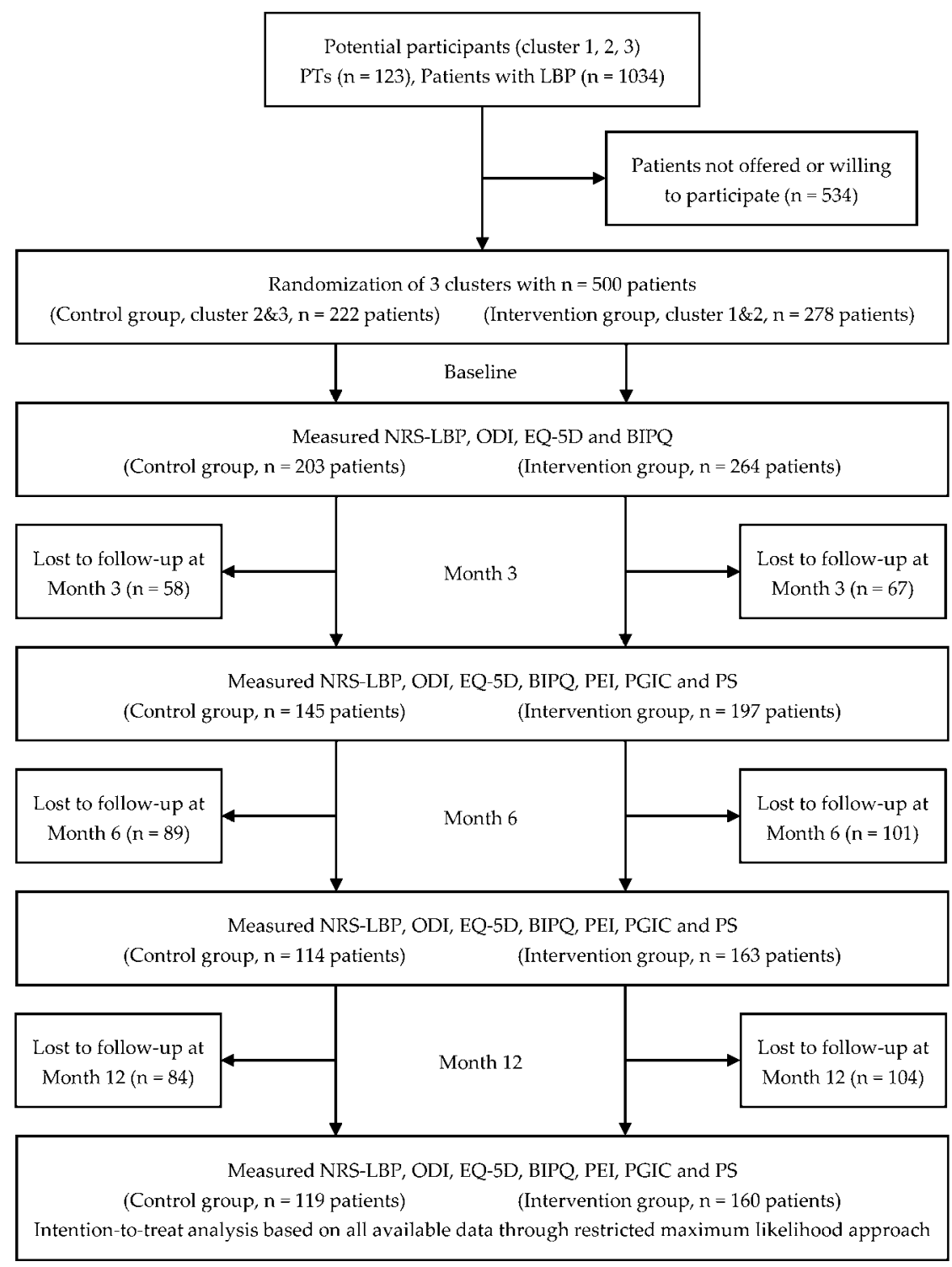

Figure 13. CONSORT flowchart of participants throughout the trial in Paper IV 
Figure 13 presents the CONSORT flow chart describing the result of recruitment and data collection. A study-specific MCID for change in primary and secondary outcomes at 3, 6 and 12 months is displayed in Table 16, and was used when evaluating PROMs.

Table 16. Minimal clinically important difference (MCID) interpreted with optimal cutoff point (OCP) at baseline to follow-ups for the total study cohort $(n=467)$

\begin{tabular}{lcccccc}
\hline \multicolumn{1}{c}{ PROMs } & $\begin{array}{c}\text { Correlation } \\
\text { with PGIC }\end{array}$ & $\begin{array}{c}\text { Change from } \\
\text { baseline } \\
\text { Mean } \pm \text { SD }\end{array}$ & $\begin{array}{c}\text { MCID } \\
\text { OCP }\end{array}$ & $\begin{array}{c}\text { Youden } \\
\text { index }\end{array}$ & $\begin{array}{c}\text { (Sensitivity; } \\
\text { Specificity) }\end{array}$ & AUC \\
\hline 3 months & & & & & & \\
ODI $(n=337)$ & 0.45 & $8.7 \pm 15.1$ & 4.5 & 0.52 & $(0.69 ; 0.83)$ & 0.81 \\
NRS-LPB $(n=337)$ & 0.39 & $2.7 \pm 2.9$ & 2.5 & 0.41 & $(0.58 ; 0.82)$ & 0.76 \\
EQ-5D index $(n=320)$ & 0.36 & $0.12 \pm 0.32$ & 0.02 & 0.39 & $(0.66 ; 0.73)$ & 0.74 \\
BIPQ $(n=339)$ & 0.52 & $8.6 \pm 16.1$ & $0.5+$ & 0.56 & $(0.81 ; 0.75)$ & 0.87 \\
PEI* $(n=335)$ & 0.50 & $4.4 \pm 4.0$ & 2.5 & 0.59 & $(0.76 ; 0.83)$ & 0.86 \\
6 months & & & & & & \\
ODI $(n=270)$ & 0.40 & $10.4 \pm 16.6$ & 4.5 & 0.44 & $(0.68 ; 0.76)$ & 0.78 \\
NRS-LPB $(n=270)$ & 0.30 & $2.6 \pm 2.9$ & 2.5 & 0.35 & $(0.59 ; 0.76)$ & 0.70 \\
EQ-5D index $(n=259)$ & 0.21 & $0.18 \pm 0.31$ & 0.03 & 0.27 & $(0.71 ; 0.56)$ & 0.67 \\
BIPQ $(n=273)$ & 0.37 & $9.4 \pm 16.0$ & 8.5 & 0.40 & $(0.58 ; 0.82)$ & 0.74 \\
PEI* $(n=265)$ & 0.48 & $4.6 \pm 4.2$ & 3.5 & 0.55 & $(0.67 ; 0.88)$ & 0.82 \\
12 months & & & & & & 0.74 \\
ODI $(n=270)$ & 0.35 & $11.9 \pm 15.6$ & 8.5 & 0.38 & $(0.59 ; 0.79)$ & 0.75 \\
NRS-LPB $(n=273)$ & 0.32 & $2.9 \pm 2.8$ & 1.5 & 0.35 & $(0.77 ; 0.58)$ & 0.72 \\
EQ-5D index $(n=264)$ & 0.31 & $0.18 \pm 0.32$ & 0.18 & 0.30 & $(0.49 ; 0.81)$ & 0.70 \\
BIPQ $(n=271)$ & 0.47 & $10.8 \pm 16.0$ & 12.5 & 0.49 & $(0.53 ; 0.98)$ & 0.82 \\
PEI* $(n=261)$ & 0.47 & $4.9 \pm 4.2$ & 2.5 & 0.53 & $(0.76 ; 0.78)$ & 0.82 \\
\hline
\end{tabular}

PROM = Patient Reported Outcome Measure, PGIC = Patient Global Impression of Change, SD = Standard Deviation, MCID = Minimal Clinical Important Difference, OCP = Optimal Cut-off Point, AUC = Area Under the Curve, ODI = Oswestry Disability Index, NRS-LBP = Numeric Rating Scale-Low Back Pain, EQ-5D = EuroQol 5 dimensions, $\mathrm{BIPQ}=$ Brief Illness Perception Questionnaire, $\mathrm{PEI}{ }^{*}=$ Patient Enablement Instrument, PEI is a transition rating scale with only 3-, 6- and 12 -month values. ${ }^{\dagger}=\mathrm{OCP}$ (0.5 ) nearest value indicating improvement on the PROM was chosen as the MCID 


\section{The effectiveness of implementation on patient-reported outcomes}

In Paper IV the effectiveness of implementation of the BetterBack:; MoC was evaluated by comparing longitudinal PROMs from patients treated after implementation (intervention $n=264$ ) with patients in routine PT care (control $n=203$ ); see Tables 17 and 18 . There were no between-group differences in the primary outcomes of change in ODI and NRS-LBP from baseline to 3 months (Table 17). However, the intervention group showed significantly higher satisfaction with care and clinically meaningful greater improvement in BIPQ at 3 months. Furthermore, the intervention group showed a clinically meaningful greater EQ-5D healthrelated quality of life at both 3- and 6-month follow-ups. Changes in PEI and PGIC did not differ between groups. Both groups displayed statistically significant and clinically meaningful within-group improvement over time, except for no clinically meaningful improvement in NRS-LBP at 6 months in the routine care group and in BIPQ at 12 months in both groups. 
Table 17. Comparisons of patient-reported outcome measures in control and intervention group

\begin{tabular}{|c|c|c|c|c|}
\hline & \multicolumn{2}{|c|}{ Within-group analysis of change from baseline } & \multirow{2}{*}{\multicolumn{2}{|c|}{$\begin{array}{l}\text { Between-group effects (1-2) } \\
\text { at each endpoint }\end{array}$}} \\
\hline & $\begin{array}{l}\text { (1) Control group } \\
(n=203)\end{array}$ & $\begin{array}{l}\text { (2) Intervention group } \\
(n=264)\end{array}$ & & \\
\hline & $\begin{array}{c}\text { Mean }(95 \% \mathrm{Cl}) \\
\mathrm{p} \text {-value }\end{array}$ & $\begin{array}{c}\text { Mean }(95 \% \mathrm{Cl}) \\
\mathrm{p} \text {-value }\end{array}$ & $\begin{array}{c}\text { Mean }(95 \% \mathrm{Cl}) \\
\mathrm{p} \text {-value }\end{array}$ & ICC \\
\hline ODI (0-100) & $31.6(27.2$ to 36.1$) *$ & $30.4(25.6 \text { to } 35.3)^{*}$ & & \\
\hline 3 months & $\begin{array}{c}-10.5(-13.4 \text { to }-7.6) \\
\mathbf{p}<\mathbf{0 . 0 0 1}\end{array}$ & $\begin{array}{c}-8.7(-11.2 \text { to }-6.2) \\
\mathbf{p}<\mathbf{0 . 0 0 1}\end{array}$ & $\begin{array}{c}-1.8(-5.0 \text { to } 1.3) \\
p=0.248\end{array}$ & 0.012 \\
\hline 6 months & $\begin{array}{c}-10.9(-14.1 \text { to }-7.7) \\
\mathbf{p}<0.001\end{array}$ & $\begin{array}{c}-10.2(-12.9 \text { to }-7.5) \\
\mathbf{p}<0.001\end{array}$ & $\begin{array}{c}-0.7(-4.2 \text { to } 2.7) \\
p=0.674\end{array}$ & \\
\hline 12 months & $\begin{array}{c}-14.2(-17.3 \text { to }-11.1) \\
\mathbf{p}<\mathbf{0 . 0 0 1}\end{array}$ & $\begin{aligned}-11.3 & (-13.9 \text { to }-8.6) \\
\mathbf{p} & <\mathbf{0 . 0 0 1}\end{aligned}$ & $\begin{array}{c}-3.0(-6.3 \text { to } 0.4) \\
p=0.081\end{array}$ & \\
\hline NRS-LBP $(0-10)$ & $6.1(5.6 \text { to } 6.7)^{*}$ & $6.4(5.7$ to 7.0$) *$ & & \\
\hline 3 months & $\begin{array}{c}-2.6(-3.1 \text { to }-2.1) \\
\mathbf{p}<0.001\end{array}$ & $\begin{array}{c}-2.9(-3.4 \text { to }-2.5) \\
\mathbf{p}<\mathbf{0 . 0 0 1}\end{array}$ & $\begin{array}{c}-0.3(-0.3 \text { to } 0.9) \\
p=0.263\end{array}$ & 0.008 \\
\hline 6 months & $\begin{array}{c}-2.4(-3.0 \text { to }-1.8) \\
\mathbf{p}<0.001\end{array}$ & $\begin{aligned}-2.7(-3.2 \text { to }-2.2) & \\
\mathbf{p} & <0.001\end{aligned}$ & $\begin{array}{c}-0.3(-0.3 \text { to } 0.9) \\
p=0.357\end{array}$ & \\
\hline 12 months & $\begin{array}{c}-3.1(-3.7 \text { to }-2.5) \\
\mathbf{p}<0.001\end{array}$ & $\begin{array}{c}-2.8(-3.3 \text { to }-2.3) \\
\mathbf{p}<0.001\end{array}$ & $\begin{array}{c}-0.3(-0.9 \text { to } 0.3) \\
p=0.297\end{array}$ & \\
\hline EQ-5D index (-0.59-1) & $0.55(0.50$ to 0.60$) *$ & 0.52 (0.46 to 0.58$) *$ & & \\
\hline 3 months & $\begin{array}{c}0.12(0.06 \text { to } 0.18) \\
\mathbf{p}<\mathbf{0 . 0 0 1}\end{array}$ & $\begin{array}{c}0.15(0.10 \text { to } 0.21) \\
\mathbf{p}<\mathbf{0 . 0 0 1}\end{array}$ & $\begin{array}{c}-0.03(-0.10 \text { to } 0.04) \\
p=0.381\end{array}$ & 0.004 \\
\hline 6 months & $\begin{array}{c}0.13(0.07 \text { to } 0.19) \\
\mathbf{p}<0.001\end{array}$ & $\begin{array}{c}0.20(0.15 \text { to } 0.25) \\
\mathbf{p}<0.001\end{array}$ & $\begin{array}{c}-0.07(-0.14 \text { to }-0.01) \\
p=0.034\end{array}$ & \\
\hline 12 months & $\begin{array}{c}0.19(0.13 \text { to } 0.25) \\
\mathbf{p}<\mathbf{0 . 0 0 1}\end{array}$ & $\begin{array}{c}0.20(0.14 \text { to } 0.25) \\
\mathbf{p}<\mathbf{0 . 0 0 1}\end{array}$ & $\begin{array}{c}-0.01(-0.07 \text { to } 0.06) \\
p=0.838\end{array}$ & \\
\hline BIPQ total score (0-80) & $44.6(40.4 \text { to } 48.8)^{*}$ & $45.4(40.7$ to 50.2$) *$ & & \\
\hline 3 months & $\begin{array}{c}-8.2(-11.4 \text { to }-5.1) \\
\mathbf{p}<0.001\end{array}$ & $\begin{array}{c}-9.0(-11.6 \text { to }-6.3) \\
\mathbf{p}<\mathbf{0 . 0 0 1}\end{array}$ & $\begin{array}{c}-0.8(-2.6 \text { to } 4.1) \\
p=0.659\end{array}$ & 0.007 \\
\hline 6 months & $\begin{array}{c}-9.1(-12.5 \text { to }-5.8) \\
\mathbf{p}<\mathbf{0 . 0 0 1}\end{array}$ & $\begin{array}{c}-8.8(-11.6 \text { to }-6.0) \\
\mathbf{p}<\mathbf{0 . 0 0 1}\end{array}$ & $\begin{array}{c}-0.2(-3.9 \text { to } 3.2) \\
p=0.853\end{array}$ & \\
\hline 12 months & $\begin{array}{c}-11.7(-15.0 \text { to }-8.4) \\
\mathbf{p}<\mathbf{0 . 0 0 1}\end{array}$ & $\begin{array}{c}-10.4(-13.2 \text { to }-7.5) \\
\mathbf{p}<0.001\end{array}$ & $\begin{array}{c}-1.3(-4.9 \text { to } 2.2) \\
p=0.457\end{array}$ & \\
\hline PEI (0-12) & $\begin{array}{l}\text { Transition score, } \\
\text { mean } \pm \text { SE }\end{array}$ & $\begin{array}{c}\text { Transition score, } \\
\text { mean } \pm \text { SE }\end{array}$ & & \\
\hline 3 months & $4.4 \pm 0.3$ & $4.5 \pm 0.3$ & $\begin{array}{c}-0.1(-1.0 \text { to } 0.7) \\
p=0.768\end{array}$ & $<0.001$ \\
\hline 6 months & $4.2 \pm 0.4$ & $4.8 \pm 0.3$ & $\begin{array}{c}-0.6(-0.4 \text { to } 1.6) \\
p=0.257\end{array}$ & \\
\hline 12 months & $5.1 \pm 0.4$ & $4.9 \pm 0.3$ & $\begin{array}{c}0.2(-1.2 \text { to } 0.9) \\
p=0.742\end{array}$ & \\
\hline
\end{tabular}

$\mathrm{n}=$ number of participants, $\mathrm{SE}=$ Standard Error, $\mathrm{Cl}=$ Confidence Interval, $\mathrm{ICC}=$ Intracluster Correlation Coefficient, ODI = Oswestry Disability Index, NRS-LBP = Numeric Rating Scale-Low Back Pain, EQ-5D = EuroQol 5 dimensions, $\mathrm{BIPQ}=$ Brief IIIness Perception Questionnaire, $\mathrm{PEI}{ }^{*}=$ Patient Enablement Instrument, $\mathrm{PEI}$ is a transition rating scale with only 3-, 6- and 12-month values. Bonferroni corrected significance thresholds of $p \leq$ 0.017 are printed in bold. $*$ Mean $(95 \% \mathrm{Cl})$ at baseline. 
Table 18. Comparisons of patient-reported experience measures in control and intervention group

\begin{tabular}{|c|c|c|c|}
\hline & (1) Control group & $\begin{array}{l}\text { (2) Intervention } \\
\text { group }\end{array}$ & Between-group comparison (2/1) \\
\hline & $\mathrm{n} / \mathrm{N}(\%)$ & $\mathrm{n} / \mathrm{N}(\%)$ & OR (95\% Cl), p-value, ICC \\
\hline \multicolumn{4}{|l|}{ Patient satisfaction } \\
\hline Satisfied after 3 months & $93 / 144(64.6)$ & 149/197 (75.6) & 1.7 (1.5 to 1.9 ), $\mathbf{p}<\mathbf{0 . 0 0 1}, \mathrm{ICC}=0.006$ \\
\hline Satisfied after 6 months & $68 / 111(61.3)$ & $109 / 163(66.9)$ & 1.3 (1.0 to 1.6$), p=0.027, I C C=0.002$ \\
\hline Satisfied after 12 months & $73 / 116(62.9)$ & $98 / 158(62.0)$ & 0.9 (0.5 to 1.6$), p=0.748, I C C<0.001$ \\
\hline \multicolumn{4}{|l|}{ Patient global rating of change } \\
\hline Improved after 3 months & $105 / 144(72.9)$ & 149/197 (75.6) & 1.2 (0.7 to 1.9 ), $p=0.570, I C C<0.001$ \\
\hline Improved after 6 months & $74 / 111(66.7)$ & $126 / 163(77.3)$ & 1.7 (1.0 to 2.9 ), $p=0.054, \mathrm{ICC}<0.001$ \\
\hline Improved after 12 months & $86 / 118$ (72.9) & $121 / 158(76.6)$ & 1.6 (0.7 to 3.9$), p=0.290, I C C=0.035$ \\
\hline
\end{tabular}

$\mathrm{n}=$ number of participants with favourable outcome, $\mathrm{N}=$ total number of participants, $\mathrm{OR}=$ Odds Ratio, $\mathrm{Cl}=$ Confidence Interval, ICC = Intracluster correlation coefficient. Bonferroni corrected significance thresholds of $p$ $\leq 0.017$ are printed in bold.

\section{Guideline adherence effect on patient-reported outcomes}

In Paper IV the five clinical practice quality indicators that formed the overall adherence in Paper III were used (Table 9). PROMs from 164 patients treated with physiotherapy where all five clinical practice quality indicators were fulfilled (CPQI-adherent care group) were compared with the non-adherent care group $(n=191)$ treated with physiotherapy where less than five clinical practice quality indicators were fulfilled. The CPQIadherent care group showed a statistically significant larger improvement in PEI throughout all follow-ups as well as NRS-LBP and BIPQ from baseline to 3 and 6 months compared with the non CPQI-adherent care group (Table 19). There was also a clinically meaningful larger improvement in the BIPQ from baseline to 3 months and in EQ-5D index from baseline to 3 and 6 months in advantage for the CPQI-adherent care group.

Table 20 presents patient-reported experience measures showing a statistically significant higher proportion of satisfaction with LBP care after 3 months (OR 2.2, 95\%CI 1.3 to 4.0, $\mathrm{p}=0.006$ ) and 6 months (OR 2.7, 95\% CI 1.5 to $6.4, \mathrm{p}=0.001$ ) in advantage for the CPQI-adherent group. PGIC was also statistically significant higher after 3 months (OR 2.3, 95\% CI 1.2 to $4.2, \mathrm{p}=0.009$ ) in the CPQI-adherent group. 
Table 19. Comparisons of patient-reported outcome measures for patients receiving CPQI adherent/non adherent care

\begin{tabular}{|c|c|c|c|c|}
\hline & \multicolumn{2}{|c|}{ Within-group analysis of change from baseline } & \multirow{2}{*}{\multicolumn{2}{|c|}{$\begin{array}{l}\text { Between-group effects (1-2) } \\
\text { at each endpoint }\end{array}$}} \\
\hline & $\begin{array}{l}\text { (1) Non CPQI adherent } \\
\text { care group }(n=191)\end{array}$ & $\begin{array}{l}\text { (2) CPQI adherent care } \\
\text { group }(n=164)\end{array}$ & & \\
\hline & $\begin{array}{l}\text { Mean }(95 \% \mathrm{Cl}) \\
\text { p-value }\end{array}$ & $\begin{array}{l}\text { Mean }(95 \% \mathrm{Cl}) \\
\text { p-value }\end{array}$ & $\begin{array}{c}\text { Mean }(95 \% \mathrm{Cl}) \\
\mathrm{p} \text {-value }\end{array}$ & ICC \\
\hline ODI (0-100) & $32.4(27.5$ to 37.3$) *$ & $28.3(23.5$ to 33.2$) *$ & & \\
\hline 3 months & $\begin{array}{c}-9.0(-11.8 \text { to }-6.2) \\
\mathbf{p}<0.001\end{array}$ & $\begin{array}{c}-11.3(-14.2 \text { to }-8.3) \\
\mathbf{p}<0.001\end{array}$ & $\begin{array}{c}2.3(-1.1 \text { to } 5.6) \\
p=0.178\end{array}$ & 0.012 \\
\hline 6 months & $\begin{array}{c}-8.9(-12.1 \text { to }-6.0) \\
\mathbf{p}<0.001\end{array}$ & $\begin{array}{c}-12.7(-16.1 \text { to }-9.4) \\
\mathbf{p}<0.001\end{array}$ & $\begin{array}{c}3.8(0.3 \text { to } 7.6) \\
p=0.048\end{array}$ & \\
\hline 12 months & $\begin{array}{c}-10.7(-13.9 \text { to }-7.6) \\
\mathbf{p}<0.001\end{array}$ & $\begin{array}{c}-13.2(-16.5 \text { to }-9.8) \\
\mathbf{p}<0.001\end{array}$ & $\begin{array}{c}2.4(-1.4 \text { to } 6.2) \\
p=0.207\end{array}$ & \\
\hline NRS-LBP (0-10) & $6.3(5.5$ to 7.1$) *$ & $6.1(5.4 \text { to } 6.9)^{*}$ & & \\
\hline 3 months & $\begin{array}{c}-2.5(-3.0 \text { to }-2.0) \\
\mathbf{p}<0.001\end{array}$ & $\begin{array}{c}-3.4(-4.0 \text { to }-2.8) \\
\mathbf{p}<0.001\end{array}$ & $\begin{array}{c}0.9(0.3 \text { to } 1.6) \\
\mathbf{p}=\mathbf{0 . 0 0 4}\end{array}$ & 0.008 \\
\hline 6 months & $\begin{array}{c}-2.1(-2.7 \text { to }-1.5) \\
\mathbf{p}<0.001\end{array}$ & $\begin{array}{c}-3.2(-3.8 \text { to }-2.6) \\
\mathbf{p}<0.001\end{array}$ & $\begin{array}{c}1.1(0.4 \text { to } 1.8) \\
\mathbf{p}=\mathbf{0 . 0 0 2}\end{array}$ & \\
\hline 12 months & $\begin{array}{c}-2.6(-3.2 \text { to }-2.0) \\
\mathbf{p}<0.001\end{array}$ & $\begin{array}{c}-3.1(-3.7 \text { to }-2.5) \\
\mathbf{p}<0.001\end{array}$ & $\begin{array}{c}0.5(-0.2 \text { to } 1.2) \\
p=0.169\end{array}$ & \\
\hline EQ-5D index $(-0.59-1)$ & $0.51(0.45 \text { to } 0.57)^{*}$ & 0.59 (0.52 to 0.65$) *$ & & \\
\hline 3 months & $\begin{array}{c}0.12(0.05 \text { to } 0.18) \\
\mathbf{p}<\mathbf{0 . 0 0 1}\end{array}$ & $\begin{array}{c}0.15(0.09 \text { to } 0.22) \\
\mathbf{p}<\mathbf{0 . 0 0 1}\end{array}$ & $\begin{array}{c}-0.03(-0.11 \text { to } 0.03) \\
p=0.294\end{array}$ & 0.004 \\
\hline 6 months & $\begin{array}{c}0.14(0.08 \text { to } 0.20) \\
\mathbf{p}<0.001\end{array}$ & $\begin{array}{c}0.19(0.13 \text { to } 0.26) \\
\mathbf{p}<0.001\end{array}$ & $\begin{array}{c}-0.05(-0.12 \text { to } 0.02) \\
p=0.161\end{array}$ & \\
\hline 12 months & $\begin{array}{c}0.19(0.13 \text { to } 0.25) \\
\mathbf{p}<0.001\end{array}$ & $\begin{array}{c}0.19(0.12 \text { to } 0.25) \\
\mathbf{p}<0.001\end{array}$ & $\begin{array}{c}0.00(-0.07 \text { to } 0.07) \\
p=0.985\end{array}$ & \\
\hline BIPQ total score (0-80) & $46.0(43.2$ to 48.8$) *$ & $43.9(41.0$ to 46.9$) *$ & & \\
\hline 3 months & $\begin{array}{c}-7.1(-10.1 \text { to }-4.1) \\
\mathbf{p}<0.001\end{array}$ & $\begin{array}{c}-12.2(-15.4 \text { to }-9.0) \\
\mathbf{p}<0.001\end{array}$ & $\begin{array}{l}5.1(1.5 \text { to } 8.6) \\
\mathbf{p}=\mathbf{0 . 0 0 6}\end{array}$ & 0.007 \\
\hline 6 months & $\begin{array}{c}-6.9(-10.1 \text { to }-3.6) \\
\mathbf{p}<0.001\end{array}$ & $\begin{array}{c}-12.8(-16.2 \text { to }-9.4) \\
\mathbf{p}<0.001\end{array}$ & $\begin{array}{c}6.0(2.1 \text { to } 9.8) \\
\mathbf{p}=\mathbf{0 . 0 0 2}\end{array}$ & \\
\hline 12 months & $\begin{array}{c}-9.3(-12.7 \text { to } 5.9) \\
\mathbf{p}<0.001\end{array}$ & $\begin{array}{c}-13.2(-16.7 \text { to }-9.6) \\
\mathbf{p}<0.001\end{array}$ & $\begin{array}{c}3.8(-0.2 \text { to } 7.8) \\
p=0.060\end{array}$ & \\
\hline PEI (0-12) & $\begin{array}{l}\text { Transition score, } \\
\text { mean } \pm \text { SE }\end{array}$ & $\begin{array}{l}\text { Transition score, } \\
\text { mean } \pm \text { SE }\end{array}$ & & \\
\hline 3 months & $4.1 \pm 0.3$ & $5.4 \pm 0.3$ & $\begin{array}{c}-1.4(-2.3 \text { to }-0.4) \\
\mathbf{p}=\mathbf{0 . 0 0 5}\end{array}$ & $<0.001$ \\
\hline 6 months & $4.1 \pm 0.4$ & $5.6 \pm 0.4$ & $\begin{array}{c}-1.6(-2.7 \text { to }-0.4) \\
\mathbf{p}=\mathbf{0 . 0 0 7}\end{array}$ & \\
\hline 12 months & $4.3 \pm 0.5$ & $5.9 \pm 0.5$ & $\begin{array}{c}-1.6(-2.7 \text { to }-0.4) \\
\mathbf{p}=0.008\end{array}$ & \\
\hline
\end{tabular}

$\mathrm{CPQI}=$ Clinical Practice Quality Index, $\mathrm{n}=$ number of participants, $\mathrm{SE}=$ Standard Error, $\mathrm{Cl}=$ Confidence Interval, $\mathrm{OR}=$ Odds Ratio, ICC = Intracluster Correlation Coefficient, ODI = Oswestry Disability Index, NRS-LBP = Numeric Rating Scale-Low Back Pain, EQ-5D = EuroQol 5 dimensions, BIPQ = Brief Illness Perception Questionnaire, $\mathrm{PEI}=$ Patient Enablement Instrument, $\mathrm{PEI}$ is transition rating scale with only 3-, 6- and 12-months values. Bonferroni corrected significance thresholds of $\mathbf{p} \leq \mathbf{0 . 0 1 7}$ are printed in bold. * Mean $(95 \% \mathrm{Cl})$ at baseline. 
Table 20. Comparisons of patient-reported experience measures for patients receiving CPQI adherent/non adherent care

\begin{tabular}{|c|c|c|c|}
\hline & $\begin{array}{l}\text { (1) Non CPQI } \\
\text { adherent care } \\
\text { group }\end{array}$ & $\begin{array}{l}\text { (2) CPQI adherent } \\
\text { care group }\end{array}$ & Between-group comparison (2/1) \\
\hline & & $\mathrm{n} / \mathrm{N}(\%)$ & OR $(95 \% \mathrm{Cl})$, p-value, ICC \\
\hline \multicolumn{4}{|l|}{ Patient satisfaction } \\
\hline Satisfied after 3 months & $90 / 138(65.2)$ & $101 / 125(80.8)$ & 2.2 (1.3 to 4.0$), \mathbf{p}=\mathbf{0 . 0 0 6}, \mathrm{ICC}=0.006$ \\
\hline Satisfied after 6 months & $62 / 110(56.4)$ & $80 / 103(77.7)$ & $2.7(1.5$ to 6.4$), \mathbf{p}=\mathbf{0 . 0 0 1}, \mathrm{ICC}=0.002$ \\
\hline Satisfied after 12 months & $58 / 104(55.8)$ & $71 / 102(69.6)$ & 1.2 (1.0 to 3.2 ), $p=0.042, I C C<0.001$ \\
\hline \multicolumn{4}{|l|}{ Patient global rating of change } \\
\hline Improved after 3 months & $98 / 138(71.0)$ & $106 / 125(84.8)$ & $2.3(1.2$ to 4.2$), \mathbf{p}=0.009, I C C<0.001$ \\
\hline Improved after 6 months & $74 / 110(67.3)$ & $84 / 104(80.8)$ & 2.0 (1.1 to 3.9 ), $p=0.027, \mathrm{ICC}<0.001$ \\
\hline Improved after 12 months & 75/106 (70.8) & 79/102 (77.5) & $1.5(0.8$ to 2.8$), p=0.206, I C C=0.035$ \\
\hline
\end{tabular}




\section{DISCUSSION}

\section{Main findings}

The implementation of a best practice physiotherapy healthcare model (BetterBack:-) MoC) for LBP improved both clinician and patient outcomes. PTs increased use of guideline-adherent care, confidence and biopsychosocial orientation in managing LBP. Patients reported improved satisfaction, illness perception and health-related quality of life. The implementation did not decrease referral to specialist consultation from an initial low level; nor did it improve patients' disability and pain more than routine care. However, when guideline-adherent care was fulfilled, most PROMs improved. The psychometric testing of the DIBQ resulted in a feasible and valid 26-item version, DIBQ-t. When DIBQ-t was used, PTs showed high expectations, which may have facilitated the implementation process initially, but the determinants of implementation behaviours decreased during the volition phase, underscoring a need of sustainability strategies for the BetterBack $\odot \mathrm{MoC}$ implementation.

\section{Evaluation of guideline implementation}

By applying a hybrid type II design in the experimental studies, we evaluated the implementation of the BetterBack:- MoC through its adoption on the PT level and the effects of clinical interventions delivered on a patient level. Moreover, patient interviews (137) and clinician interviews (unpublished data) have also been performed, but these are not included in this thesis. These evaluations can help determine whether the LBP care, after a multifaceted implementation of a best practice healthcare model, can improve in quality. However, there are other ways to measure quality and fidelity. We have not evaluated how and with what quality, dose or amount the actual components of the BetterBack $\odot$ MoC were delivered. According to suggested implementation outcomes by Proctors et al. (84), cost and sustainability evaluations are not covered. In line with the published study protocol, analysis of implementation costs and patientrelated healthcare costs are planned but not presented in this thesis. Moreover, the longitudinal evaluation was up to one year after the implementation, making it difficult to determine to what extent the BetterBack $;$ MoC is incorporated in routine practice beyond the first year of implementation. 


\section{The implementation intervention}

The implementation of the BetterBack $;$ MoC showed an improvement in PTs' self-confidence in diagnosing and treating patients with LBP. Furthermore, changed attitudes and beliefs from a more biomedical towards a more evidence-based biopsychosocial treatment orientation was found (Paper II). Changes in attitudes and beliefs after various interventions in PT contexts have been shown by others $(55,138,139)$. Three studies showed similar results to our study (139-141). This was in a voluntary university course with follow-up six months after the course (140) as well a short seven-hour workshop with follow-ups directly after the workshop (139) and a qualitative study involving PTs from four countries participating in intensive biopsychosocial training (141) However, in these studies the follow-ups took place between immediately after the course/training up to six months after, with small PT cohorts (12 - 42 PTs) and with potential selection bias towards motivated participants (139-141). Several studies have demonstrated that HCPs' attitudes and beliefs are associated with the treatment they give to their patients with LBP (55), but this does not systematically translate into behaviour and practice change (51). There are few studies that demonstrate an actual change in treatment behaviour $(51,142,143)$, and even fewer showing improvement of patient-reported outcomes after implementation in PT contexts $(51,144)$.

In a scoping review, seven of nine studies showed increased PT adherence to CPGs as a result of knowledge translation strategies (142). However only three involved LBP CPGs $(57,143,145)$. Only the RCT by Bekkering et al (143) had a similar design as our study, using individual patients' forms to record treatment, while Van Dulmen et al. (145) used vignettes to evaluate adherence and Bernhardsson et al. (57) evaluated PTs' self-reported guideline use through a single question. Our results show a behaviour change in terms of improved guideline-adherent care: a significant 3.5 times higher likelihood, equals $33.2 \%$ increase of receiving guideline-adherent care delivered by PTs after implementation of the BetterBack $;$ MoC, compared to the routine care group. Earlier research has found a smaller increase ranging from $3.1 \%$ to $12 \%$ regarding adherence to LBP CPG after PTs have received CPG education (143, 145147). However, these studies used varying ways of measuring overall adherence, making exact comparisons of study results difficult.

The use of the BetterBack $;$ MoC's core component patient information/education showed the largest between-group differences, with a 5.1 times greater likelihood of receiving this treatment in the intervention group compared to routine care group (Paper III). There was also a tendency towards stratified use of educational interventions in the 
intervention group: patients in medium/high risk groups were 8.1 times more likely to receive educational interventions compared to routine care. Increased use of patient education after PT guideline implementation has also been shown by Bekkering et al. (143), but to our knowledge we are the first to show a stratification with a larger proportion of use in patients in STB medium/high risk of LBP persistence after implementation of the BetterBack $\odot$ MoC. A qualitative review showed that PTs feel unprepared to identify and treat cognitive, social and psychological factors in LBP, and recommend use of the SBT to identify patients at risk, and the use of education and training to influence these factors (148). This is also supported by a systematic review including five RCTs of PT-delivered cognitive-behavioural interventions for LBP, concluding that without training and resources, successful translation and implementation remain unlikely (53) The BetterBack@) MoC offers PTs easy tools for stratification, information and education and training on how to use these tools. Risk stratification tools have previously not been used routinely in Swedish primary care physiotherapy. Lack of time in clinical practice has been reported as the number one barrier for Swedish PTs to use CPGs (2).

Stratification of number of PT visits showed small but significant differences between groups: $54.7 \%$ in the intervention vs $44.6 \%$ in the routine care group. This may be explained by the lower clinical priority ranking of this CPG recommendation by the MoC support team in the development phase. Furthermore, due to logistical reasons in some clinics, the STB questionnaire was delivered and collected by reception staff, which made PTs unable to utilise the results to plan intervention delivery. Literature on the adherence to CPG recommendations regarding the number of PT clinical visits has been reported in two previous studies (143, 149). These reported on similar guidelines with a maximum of 3 treatment sessions for patients with benign LBP of less than 3 weeks' pain duration, showing only $17 \%$ to $20 \%$ adherence to this recommendation $(143,149)$. Consensus concerning the optimal number of visits is however difficult to establish, and may require adaptation to the specific health care system context.

The proportions of patients receiving manual therapy or acupuncture according to guidelines were low and similar in both groups. This can be explained by a more prominent focus of active treatments on improving patient's self-management strategies in the MoC. The CPG recommendation for use of exercise was already high in the routine care group (>88\%), with no group differences compared to after the BetterBack $;$ MoC implementation, indicating that this recommendation was already fulfilled, with little room for improvement. This is in line with another Swedish primary care study which reported $82 \%$ use of exercise for LBP (3). Having little room for improvement was also the case for our 
primary outcome regarding referral to specialist care, where only $2.2 \%$ of referrals were registered during the PT treatment period in the intervention group vs $5.4 \%$ in the routine care group, with no between-group differences. The initial reasoning for choosing referrals to specialist care as a primary outcome was based on high numbers of these referrals in the region of Östergötland. However, this result suggests that these referrals were most likely made by other healthcare professionals such as general practitioners. These low referral rates are in line with findings that patients with musculoskeletal disorders treated by PTs were less often referred to secondary care compared to patients treated by general practitioners (150). To change this outcome it may be necessary to involve all HCPs who are gatekeepers for referrals. The overall quality index including the five core CPG recommendations would have been a better choice of primary outcome in the current study and can be suggested in similar healthcare settings. In summary, our findings show a more frequent use of active treatment strategies. This is in line with how primary care physiotherapy for LBP has developed from a larger focus on passive strategies historically to more active strategies at the present (1).

The proportion of patients receiving medical imaging during the PT treatment period was significantly lower, with $8.3 \%$ in the intervention versus $16.3 \%$ in the routine care group. In Swedish primary care referrals to medical imaging are mainly done by general practitioners. However, the implementation may have influenced patients that routine imaging is not necessary during the PT treatment period. Despite decades of guideline recommendations with advice to decrease imaging for benign LBP, the prevalence is still constant and too high, with a medical imaging rate of $25 \%$ for LBP cases in primary care (18). This high prevalence indicates a need to change HCPs' and patients' beliefs that routine imaging is necessary. To support this change, educational interventions have been suggested (151).

A successful implementation with the aim of improving quality of care needs to include both increased use of evidence-based and decreased use of non-evidence-based treatments. In Paper III, we could show a significantly decreased use of non-evidence-based PT treatments and medical imaging in the intervention group indicating that the BetterBack $:-$ $\mathrm{MoC}$ could contribute to both implementation and de-implementation of physiotherapy interventions.

\section{Behaviour change is time dependent}

Changing all PTs' treatment behaviour within the study period may not be realistic. Earlier research in PT contexts has found a small increase of adherence to LBP CPGs, ranging from $3.1 \%$ to $12 \%$ increase of adherence to LBP CPGs after various CPG educational interventions (143, 145-147), compared to a $33.3 \%$ improvement over all adherence in our study. This 
together with the findings that $41 \%$ of patients still received non-adherent care in the intervention group shows that we were not able to change all PTs' behaviour. Behaviour change is time-dependent. According to the diffusion theory (61), people in a group vary from "early adopters" to "laggers", and laggers may not have had enough time to adopt to the BetterBack@ MoC during our short patient recruitment period. Also, a substantial change of attitudes and beliefs among PTs may take time. We did not find a significant change towards a biopsychosocial orientation until 12 months after the start of the BetterBack:- MoC implementation strategy, indicating that longer follow-ups may be needed to identify whether behaviour change has occurred.

\section{Desired behaviour can already exist}

In Paper III we could show that the adherence to the five core recommendations in the overall CPQI was also evident in the routine care group, with $26 \%$ of patients receiving care that fulfilled the overall CPQI. Bekkering et al. (143) have in a randomised trial also shown that a proportion of the control group was already in compliance with CPG recommendations. A previous non-randomised Swedish guideline implementation study including primary-care PTs in another healthcare region concluded that treatment methods were largely in line with evidence before the implementation, which explained why the guideline implementation had little impact on clinical practice (152).

\section{Improved adherence is needed}

Despite significant improvement in the PT delivery of guidelineadherent care, with $59 \%$ of patients receiving care that adhered to all five core recommendations after implementation compared to $26 \%$ in the routine care group , there were still $41 \%$ of patients receiving non-adherent care in the intervention group. These findings can contribute with understanding why the effectiveness of implementation of BetterBack:-) MoC on PROMs was small and diluted, and gave us the idea of the additional exploratory analyses with two new groups: CPQI-adherent care group and the non-CPQI-adherent care group showing a greater improvement in most PROMs. These findings also indicate a need to further improve guideline uptake.

\section{The multifaceted implementation strategy}

In the development and implementation of the BetterBack@; MoC we have used a framework adapted for implementation of musculoskeletal MoC (75), theories and models to plan implementation, implement, evaluate and report results $(67,68,70,72,73)$. This together with experiences from implementation of evidence-based management of 
shoulder conditions in the region of Östergötland aimed at the same PT group (153) strengthens the implementation strategy. A further strength of our implementation strategy has been the use of top-down support from managers and bottom-up support from selected clinical champions involved in the development, implementation and sustainability phases. However, after implementation, 41\% of PTs still did not deliver care according to the five core recommendations in the overall CPQI (Paper III). Furthermore, results from Paper IV show a decreasing longitudinal trend in most facilitating determinants of implementation behaviours at three months after the implementation workshop. These trends indicate that additional facets in the implementation strategy are needed after the initial implementation phase. For example, a use of the DIBQ-t with rapid analysis and actions on the findings could have provided more support during the sustainability phase of the multifaceted implementation strategy. We introduced an online chat forum to allow communication between PTs and researchers. However, this chat forum was rarely used and could have been promoted more effectively. During outreach visits 7 to 12 months after implementation, we conducted SWOT analyses to address potential weaknesses, threats and highlight strengths and opportunities of the MoC. This was an effective way to troubleshoot, and it allowed feedback and discussions between researchers and PTs. However, to sustain the use of the BetterBack $:$ MoC, this might not have been enough.

Changing clinicians' behaviours is challenging. Multiple barriers have been suggested, for instance that some clinicians consider guidelines as a threat to their professional autonomy, and that deviating from wellestablished habits is challenging when time constraints are present (48). A lack of support from managers, and patients' role expectations on the PT are other barriers in clinical practice that have been reported (47). In this thesis, we have not directly asked individual PTs to report actual barriers, but instead we have evaluated PTs' rating of specific determinants of their implementation behaviours which can be seen as potential barriers and facilitators for implementation success/failure. PTs had high expectations of the MoC directly after the workshop. Most determinants decreased after real-world practice but they still displayed a facilitating pattern during the first year after implementation. This indicates that PT initially high expectations of the $\mathrm{MoC}$ may facilitate the implementation process to start with, but there is a need for sustainability strategies after the initial implementation. The current multifaceted implementation strategy, including well-planned guideline development, education, manager support and use of clinical champions, gave good effects and mostly facilitated the implementation process. However, it did not fully meet the demands on organisational and social influence levels since these domains decreased over time. A regular focus on organisational support, and 
support from clinical champions over time would probably be required in order to maintain a faciliatory effect. A recently published study with a mixed-method parallel design by the Danish research team that we collaborated with in Study A has after implementing a care package for LBP with similar content to BetterBack $(-)$ MoC showed three themes important for implementation among clinicians: personal gain, practicalities and buying-in on the programme (154). This study used DIBQ-t six months after the workshop education and found that both implementers and nonimplementers reported high levels of knowledge and skills, indicating that training alone is insufficient for implementation (154).

\section{Evaluation of the clinical intervention}

\section{Patient-reported outcomes}

When evaluating implementation, it is important to also focus on the end receiver, which in healthcare is the patient. Implementation studies evaluating PROMs are scarce in the PT context, and few studies have been able to show improvement in PROMs $(51,144,155,156)$. A systematic review found only eight studies that evaluated various implementation strategies in the LBP physiotherapy contexts (51). Five of these studies were considered to have good quality and of these five, only one could show improved PROMs after a single-faceted implementation of a stratified primary care model (157). However, when this study was replicated with a larger cohort ( $\mathrm{n}=1701$ patients), no significant differences could be seen in patient outcomes (158). Multiple reasons for this difficulty in showing improvements in PROMs have been suggested, such as low quality and quantity of the implementation strategy aimed at changing practice (144). Furthermore, the natural course of LBP, with a pattern of flare ups as well as rapid and substantial improvements, may also limit potential effects to the short term in LBP studies as also seen in Paper IV (159). This together with the earlier discussed need of sustainability strategies for implementation at PT level (51) may add to the complicated evaluation of PROMs.

In Paper IV we could not show better primary or secondary outcomes after implementation of the BetterBack $\odot$ MoC (intervention), compared to routine care (control) except for significant improvements of patient satisfaction with LBP care at 3 months as well as short-term clinically meaningful improvements in quality of life and LBP illness perception. A higher satisfaction in the intervention group can be explained by the slightly larger number of PT treatment sessions with a mean of 4.6 (SD 3.8) sessions in the intervention group compared to 3.1 (SD 2.7) sessions in the control group. However, the larger number of treatments in the 
intervention group can be explained by the more often used group training that yields a higher number of treatment sessions. High satisfaction with LBP care has also been shown in a qualitative study that explored patients' experience of the BetterBack:-) MoC, where patients experienced better knowledge of their LBP and had received tools to better manage their health condition (137). Patient satisfaction with care has been seen to correlate with quality of life, and it has been suggested that although changes in treatment of patients might not lead to significant changes in patients' function and activities, clinicians can potentially affect patients' perception of LBP and improve coping with illness (160).

Based on our findings which showed that $26 \%$ of the control group already provided guideline-adherent care compared to $59 \%$ after the BetterBack $\odot$ MoC implementation in the intervention group, a secondary explorative analysis was performed. This secondary analysis showed that PTs' $^{\prime}$ adherence to all five core recommendations (CPQI-adherent care) significantly improved most PROMs, compared to non-CPQI-adherent care. Fritz et al. (161), using a retrospective case control design, found that care adherent to LBP guidelines was associated with higher improvement of pain and disability than non-adherent care. However, similar improvements in disability were not shown in our study, which may be explained by our lower baseline values in our study. But with a stronger design we also evaluated other patient outcomes and had significant group differences with greater improvements in LBP illness perceptions, patient enablement, satisfaction with care and global improvement in patients receiving CPQI-adherent care.

In Paper IV, study-specific MCIDs showed that smaller changes are of importance in our patient groups compared to previously reported MCID for NRS-LBP and ODI in other populations (162). When using the MCIDs in the secondary analyses, only the outcome LBP illness perception was over the cut-off for our study specific MCID in the short term. However, the study specific MCID for EQ-5D at six-month follow-up had its weakness as it did not reach the predefined correlation of $\geq 0.3$ with the PGIC and model accuracy AUC $\geq 0.7$, indicating that this clinically meaningful improvement should be interpreted with caution. Nevertheless, core elements in BetterBack:- MoC are information and education with the aim of reducing maladaptive illness perceptions, which may explain why the improvements in illness perception were the most prominent result.

\section{The content of the BetterBack (-) MoC}

The content of the clinical intervention, including support tools for patients and PTs such as: brochure, group education, exercise resources, patient-centred coordinated pathways, risk stratification and matching treatment towards risk of persistent disability pain outcomes, was 
developed in collaboration with a Danish research group and was reviewed and tailored by the clinical champions and a spinal surgeon. This thesis has not evaluated which specific components of the clinical intervention that were effective in changing PROMs. However, according to a qualitative interview study not included in this thesis, the patients reported that the brochure, group education and exercise programme were helpful tools in developing self- management strategies, and reducing fear avoidance and maladaptive illness beliefs (137).

\section{Instrument development}

The tailoring of DIBQ resulted in a shorter 28-item questionnaire to assess clinicians' expectations of implementation. The questionnaire represented 10 of initially available 18 DIBQ domains. After feasibility and construct validity testing, a feasible and valid 26-item questionnaire was developed to be used in evaluation of best practice LBP programmes (Study A). This version was then rephrased to be used for follow-ups during the first year of implementation (Paper II). In the expectation phase (before implementation) the DIBQ-t can be regarded more as an instrument to measure readiness for change. Further testing is needed to determine the value of using DIBQ-t for follow-ups. However, a recent study showed that implementers responded more positively to most domains of the DIBQ-t, compared with non-implementers strengthening a longitudinal use of the DIBQ-t (154). In Paper II, PTs identified few barriers in the expectation phase and more barriers after they started to use the $\mathrm{MoC}$, which supports the use of DIBQ-t longitudinally to handle identified barriers. With instant analysis and action in an ongoing circular process instead of the linear process applied in our study, the implementation process could become more effective. While the DIBQ addressed clinicians' views of implementation, it is also important to evaluate the views of other, such as managers' views on service outcomes and patients' views on implementation (84).

\section{Methodological considerations}

\section{Strengths and limitations}

The randomised design, with an intervention group and a routine care group as control, is one of this thesis' greatest strength. Furthermore, the hybrid design has its strength in a dual testing of clinical and implementation intervention. This design allows a more rapid translation of research findings into routine practice, more effective implementation interventions, and more useful information for decision makers $(64,163)$. 
However, hybrid designs are typically more complex to execute and might not be feasible or affordable on a limited research budget (64). Moreover, the samples used were large enough, according to our a-priori sample calculations. To perform a CFA analysis, a sample of more than 100 is suggested (164), a sample of almost 600 clinicians was used in the methodological study (Study A). This together with a sample size for clinician and patient outcomes in Papers II- IV, which exceeded the sample size requirements, strengthens the results in this thesis. A further strength was that PTs and patients in the intervention and control groups were similar in baseline characteristics. Furthermore, the three clusters were similar, with a mix of small to large practices sizes in both rural and urban areas. These similar baseline characteristics and the mix of PTs in the clusters minimised potential selection bias.

Data on patients' physiotherapy treatments were based on PTs selfreported treatment choice and can be inaccurate due to recall bias, misconception, and lack of time to fill in the rather complicated questionnaire. A more extensive piloting of this PT questionnaire (CRPE) could have prevented this potential inaccuracy. However, this inaccuracy is likely to be similar in both groups, and offers a more authentic assessment material than more commonly used clinical vignettes used in similar research $(145,147,165,166)$.

Another strength is that complete register data were used for number of PT treatment sessions, medical imaging, and referrals to specialist consultations in addition to PT-reported CRPE. This resulted in a larger patient cohort and good quality data. However, as discussed earlier, another primary outcome than referral to specialist consultation due to low referrals in both groups could be suggested. A strength is that the CPQI was based on the highest clinical priority rankings from the MoC support team, which BetterBack:- MoC had the strongest focus on.

With regards to PROMs used in Paper IV, a strength was that the questionnaires had previously been tested for validity and reliability, and were recommended for evaluation of LBP in clinical trials (167). Our primary outcome ODI and NRS-LBP have been recommended as core outcome for clinical trials in non-specific LBP by international consensus (168). However, use of a single item to assess patient satisfaction has been questioned (117). The six-item questionnaire TOSQ-S with acceptable psychometric, also translated into Swedish, may have been a better choice to measure treatment outcome satisfaction of physiotherapy in patients with LBP (117).

Two major strengths in Paper II are the response rate and the involvement of both motivated and unmotivated PTs, with a response rate of $96 \%$ of all educated PTs employed in the public healthcare region. This was possible due to support from managers in making the implementation 
workshop mandatory. The questionnaires were distributed to participating PTs by the researchers before and directly after the workshop training. This might have resulted in response bias with more positive answers to please the researchers, while after the workshop, questionnaires may have been completed more quickly due to fatigue after a whole day of workshop. However, with allocated time to fill in the questionnaires, we were able to reach our high response rate. Other efforts to implement guidelines in Sweden reached response rates between $57 \%$ and $65 \%$ of all PTs $(3,57)$ and with even lower response rates internationally (169).

In the stepped RCT we reached $48 \%$ (Paper III) and $45 \%$ (Paper IV) of all patients seeking physiotherapy in publicly financed care during the study period in the region. Most studies do not report and are probably not aware of the total patient flow in a healthcare system. To reach almost half of all patients during the study period can be consider a good reach. Most patients not included in the stepped RCT never got an offer to participate. For these patients no data are available, and therefore we do not know how and if they differed in baseline characteristics and would respond differently compared to the included patients. A systematic registration of these patients who denied participation could have enabled an analysis of these patients.

Bonferroni correction to avoid type 1 error and the use of clustering effects to compensate for type 2 error contribute to a solid interpretation of data. However, the ICC-values were low, indicating that this compensation might not be needed, resulting in analyses with more than enough patients. A strength of the design is the use of the intention to treat principle to enable imputation of patient data when questionnaires were lost to followups. The advantages of maximum likelihood imputation are: 1) it results in unbiased estimates and provides more validity than ad-hoc approaches to missing data; and 2) it uses all available data and therefore preserves sample size and statistical power (170). It is well known that disability and pain fluctuate over time in $\operatorname{LBP}(11,12)$, therefore imputed data might not reflect this variation. However this is likely to be similar in both groups.

A weakness in Paper II was the use of a longitudinal evaluation with DIBQ when the psychometrics of this tailored longitudinal version had not yet been evaluated. A more well-evaluated questionnaire could have been a better choice but was lacking at the time. Another weakness is that the transition to routine care to intervention did not have a wash out period, however PTs were advised to not introduce the BetterBack-; MoC to patients that had been included during the routine care period. 


\section{Generalisability}

The PTs involved in the experimental studies (Papers II-IV) came from both small primary care practices with 1-4 PTs to larger practices with more than 20 PTs, and from both rural and urban areas in the region of Östergötland. The region of Östergötland has educational, income and health levels that are comparable to the whole country (171). Only publicly funded PTs participated in these studies. This makes our findings generalisable to Swedish publicly funded physiotherapy in primary care and to similar healthcare systems internationally. The methodological study (Study A) also involved both private PTs and chiropractors from Denmark, making findings from this study also generalisable to private physiotherapy and chiropractic. The implementation intervention of LBP guidelines could probably be generalised to other guideline implementation in primary care physiotherapy. Patients' characteristics in the stepped RCT were similar to those reported in other primary settings (172) and similar to an earlier study in the same primary care setting (173). One drawback common in research concerns the inclusion criterion for patients, fluency in Swedish, which may limit the generalisability. Furthermore, generalisability must be interpreted with caution since implementation success depends on a high degree of contextual factors (50). Factors suggested to be involved in implementation success are organisational support, social relations, leadership and organisational culture and climate (174). 


\section{CONCLUSIONS}

\section{Overall conclusions}

The implementation of a best practice physiotherapy healthcare model (BetterBack $;$ MoC) for LBP improved both clinician and patient outcomes. PTs increased use of guideline-adherent care, confidence and biopsychosocial orientation in managing LBP. Patients reported improved satisfaction, illness perception and health-related quality of life. The implementation did not decrease referral to specialist consultation from an initial low level; nor did it improve patients' disability and pain more than routine care. However, when guideline-adherent care was fulfilled, most PROMs improved. It is therefore important to highlight the importance of guideline-based primary care for improving patient-reported LBP outcomes.

\section{Specific conclusions}

- The 26-item DIBQ-t is a feasible and valid version of a questionnaire for evaluating clinicians' expectations regarding implementation determinants of best-practice LBP primary care programmes.

- PTs' reported implementation behaviour was a facilitator for the implementation process during the first year of implementation. However, the initially high expectation for determinants of implementation of the $\mathrm{MoC}$ may initially facilitate the implementation process, but had a decreasing trend with real-world practice, indicating a need for improved sustainability strategies after the initial implementation.

- A multifaceted implementation of a best practice PT healthcare model of care improved clinician outcomes such as PTs' confidence in diagnosing and treating patients with LBP, and changed PTs' attitudes and beliefs towards a more evidence-based biopsychosocial treatment orientation in managing patients with LBP. This may contribute to an actual change of treatment behaviour.

- The implementation of a best practice PT healthcare model effectively improved the delivery of LBP guideline-adherent care among PTs in primary care with a significant 3.5 times higher likelihood of receiving guideline-adherent care after implementation, compared to routine care. This increase of PT adherence to LBP CPGs is greater than in earlier research. A significantly improved use of stratification of number of visits and 
patient educational interventions, maintained high use of exercise and a decrease in non-evidence-based recommendation and routine medical imaging were achieved. However, the primary outcome, i.e. referral to special consultation, was low in both groups and did not differ between groups.

- The implementation of a best practice PT healthcare model resulted in a significant 1.7 times larger improvement in patient satisfaction with care and a larger clinically meaningful improvement in illness perception and health-related quality of life, compared with routine care. However, there were no group differences in the primary outcomes pain and disability or the secondary outcomes patient enablement and global impression of change. Nevertheless, patients who had received PT-delivered LBP care that fulfilled all five clinical practice indices showed greater improvement in most PROMs, compared to non-fulfilment. This indicates the importance of guideline-based primary care in improving patient-reported LBP outcomes.

\section{Clinical implications}

- Use of guideline-adherent care with the core components patient education, exercise and no use of non-evidence-based physiotherapy should be considered, in order to optimize patientreported outcomes and improve quality of LBP care.

- Easy-access support tools for PTs such as the SBT, clinical process and reasoning pathways, and structured assessment proformas can be recommended for use in primary care physiotherapy.

- Support tools targeting patients, such as an information brochure about LBP, group-based education/information, and exercise resources can be used to change patients' maladaptive illness beliefs and improve patients' satisfaction with LBP care and healthrelated quality of life.

\section{Implications for implementation efforts}

Implementation research in the field of LBP is scarce, especially regarding evaluation of implementation effects on clinicians' behaviour change and patient-reported outcomes. This thesis contributes to filling the gap regarding efforts in implementation of a best practice physiotherapy healthcare model for LBP in primary care, using both clinician and patient evaluations. Furthermore, the results of this thesis may have an impact on 
the direction of future implementation research and quality improvement of LBP care.

A multifaceted implementation strategy with both top-down and bottom-up strategies seems to be effective in increasing guideline-adherent care, improving clinician-reported confidence, attitudes and beliefs in managing LBP, and improving patient-reported satisfaction, illness perception and health-related quality of life. A multifaceted strategy is therefore suggested to be a part of an effective guideline implementation intervention. The top-down strategy with support from the rehabilitation managers during the development and implementation phases facilitated the initial implementation process. Moreover, the rehabilitation managers made the BetterBack:- MoC workshops mandatory for all PTs. This mandatory participation of PTs may have contributed to facilitate the initial implementation of the MoC. The mandatory participation also led to the collection of rich data from both motivated and unmotivated PTs, thereby reflecting real world practice.

The bottom-up strategy included selected trusted 'clinical champions' with special skills in LBP from each unit involved in the development. These clinical champions also acted as local ambassadors, which seems to be effective both to make the implementation successful and to sustain the model of care during the first year after implementation. However, despite this multifaceted strategy, $41 \%$ of patients still received care that did not adhere to all five clinical practice quality indices, and a decrease in PTs' implementation behaviours after using the model of care indicated that further uptake was needed. To improve guideline uptake, social and organisational support after the initial implementation seems to be important, and needs regular focus during an implementation process.

Evaluation of improvement of patient outcomes after an implementation intervention is challenging but needed. Few studies in physiotherapy have been able to improve patient outcomes more after an implementation intervention, compared with a control group (51). A 100\% uptake of what you as a researcher try to implement is not realistic. Therefore, when evaluating patient outcomes after guideline implementation, it is important to simultaneously evaluate if the desired treatment behaviour already exists among delivering clinicians before implementation and to what extent the behaviour has changed due to implementation.

\section{Future research}

- The DIBQ-t needs further psychometric testing - especially for longitudinally evaluation. Furthermore, studies are needed to 
determine if this questionnaire is suitable for evaluating implementation of MoC, or programmes for other health conditions and other healthcare contexts.

- There is a need to broaden the BetterBack - MoC to include both primary and secondary care, with the involvement of other important healthcare practitioners such as general practitioners, nurses, and spinal surgeons, in order to cover the whole healthcare pathway for patients with LBP. With this broader MoC, medical imaging and specialist consultation can be optimised and be reserved mainly to patients with red flags. This broad $\mathrm{MoC}$ needs to be developed, implemented and thoroughly evaluated regarding its ability to further optimise LBP care.

- Implementation strategies as well as the content used in the implementation of the BetterBack $:$ MoC need to be adapted to new digital possibilities and solutions in modern healthcare. Digitalised implementation strategies and content might improve clinician and patient uptake, and improve the sustainability of the MoC. However, this needs to be tested and evaluated. 


\section{REFERENCES}

1. Groenendijk JJ, Swinkels IC, de Bakker D, Dekker J, van den Ende $\mathrm{CH}$. Physical therapy management of low back pain has changed. Health Policy. 2007;80(3):492-9.

2. Bernhardsson S, Johansson K, Nilsen P, Oberg B, Larsson ME. Determinants of guideline use in primary care physical therapy: a cross-sectional survey of attitudes, knowledge, and behavior. Phys Ther. 2014;94(3):343-54.

3. Bernhardsson S, Oberg B, Johansson K, Nilsen P, Larsson ME. Clinical practice in line with evidence? A survey among primary care physiotherapists in western Sweden. J Eval Clin Pract. 2015;21(6):1169-77.

4. Lindback Y, Tropp H, Enthoven P, Abbott A, Oberg B. PREPARE: presurgery physiotherapy for patients with degenerative lumbar spine disorder: a randomized controlled trial. Spine J. 2018;18(8):1347-55.

5. Kongsted A, Ris I, Kjaer P, Vach W, Morso L, Hartvigsen J. GLA:D((R)) Back: implementation of group-based patient education integrated with exercises to support self-management of back pain protocol for a hybrid effectiveness-implementation study. BMC Musculoskelet Disord. 2019;20(1):85.

6. Hartvigsen J, Hancock MJ, Kongsted A, Louw Q, Ferreira ML, Genevay S, et al. What low back pain is and why we need to pay attention. Lancet. 2018;391:2356-67.

7. Hoy D, Bain C, Williams G, March L, Brooks P, Blyth F, et al. A systematic review of the global prevalence of low back pain. Arthritis Rheum. 2012;64(6):2028-37.

8. Amundsen PA, Evans DW, Rajendran D, Bright P, Bjørkli T, Eldridge $\mathrm{S}$, et al. Inclusion and exclusion criteria used in non-specific low back pain trials: a review of randomised controlled trials published between 2006 and 2012. BMC Musculoskelet Disord. 2018;19(1):113.

9. Dionne CE, Dunn KM, Croft PR, Nachemson AL, Buchbinder R, Walker BF, et al. A consensus approach toward the standardization of back pain definitions for use in prevalence studies. Spine (Phila Pa 1976). 2008;33(1):95-103.

10. Delitto A, George SZ, Van Dillen LR, Whitman JM, Sowa G, Shekelle P, et al. Low back pain. J Orthop Sports Phys Ther. 2012;42(4):A1-57.

11. Kongsted A, Kent P, Axen I, Downie AS, Dunn KM. What have we learned from ten years of trajectory research in low back pain? BMC Musculoskelet Disord. 2016;17:220.

12. Costa N, Ferreira ML, Setchell J, Makovey J, Dekroo T, Downie A, et al. A Definition of "Flare" in Low Back Pain: A Multiphase Process Involving Perspectives of Individuals With Low Back Pain and Expert Consensus. J Pain. 2019;20(11):1267-75.

13. Koes BW, van Tulder MW, Thomas S. Diagnosis and treatment of low back pain. Bmj. 2006;332(7555):1430-4. 
14. Henschke N, Maher CG, Ostelo RW, de Vet HC, Macaskill P, Irwig L. Red flags to screen for malignancy in patients with low-back pain. Cochrane Database Syst Rev. 2013(2):Cdoo8686.

15. Williams CM, Henschke N, Maher CG, van Tulder MW, Koes BW, Macaskill P, et al. Red flags to screen for vertebral fracture in patients presenting with low-back pain. Cochrane Database Syst Rev. 2013(1):Cdoo8643.

16. Konstantinou K, Dunn KM. Sciatica: review of epidemiological studies and prevalence estimates. Spine (Phila Pa 1976). 2008;33(22):246472.

17. Yabuki S, Fukumori N, Takegami M, Onishi Y, Otani K, Sekiguchi M, et al. Prevalence of lumbar spinal stenosis, using the diagnostic support tool, and correlated factors in Japan: a population-based study. J Orthop Sci. 2013;18(6):893-900.

18. Downie A, Hancock M, Jenkins H, Buchbinder R, Harris I, Underwood $\mathrm{M}$, et al. How common is imaging for low back pain in primary and emergency care? Systematic review and meta-analysis of over 4 million imaging requests across 21 years. Br J Sports Med. 2020;54(11):64251.

19. Brinjikji W, Diehn FE, Jarvik JG, Carr CM, Kallmes DF, Murad MH, et al. MRI Findings of Disc Degeneration are More Prevalent in Adults with Low Back Pain than in Asymptomatic Controls: A Systematic Review and Meta-Analysis. AJNR Am J Neuroradiol. 2015;36(12):2394-9.

20. Henschke N, Kamper SJ, Maher CG. The epidemiology and economic consequences of pain. Mayo Clin Proc. 2015;90(1):139-47.

21. Meucci RD, Fassa AG, Faria NM. Prevalence of chronic low back pain: systematic review. Rev Saude Publica. 2015;49:1-73.

22. Dagenais S, Caro J, Haldeman S. A systematic review of low back pain cost of illness studies in the United States and internationally. Spine J. 2008;8(1):8-20.

23. Jöud A, Petersson IF, Englund M. Low back pain: epidemiology of consultations. Arthritis Care Res (Hoboken). 2012;64(7):1084-8.

24. Jordan KP, Jöud A, Bergknut C, Croft P, Edwards JJ, Peat G, et al. International comparisons of the consultation prevalence of musculoskeletal conditions using population-based healthcare data from England and Sweden. Ann Rheum Dis. 2014;73(1):212-8.

25. Richmond J. Multi-factorial causative model for back pain management; relating causative factors and mechanisms to injury presentations and designing time- and cost effective treatment thereof. Med Hypotheses. 2012;79(2):232-40.

26. Pincus T, Kent P, Bronfort G, Loisel P, Pransky G, Hartvigsen J. Twenty-five years with the biopsychosocial model of low back pain-is it time to celebrate? A report from the twelfth international forum for primary care research on low back pain. Spine (Phila Pa 1976). 2013;38(24):2118-23.

27. Mescouto K, Olson RE, Hodges PW, Setchell J. A critical review of the biopsychosocial model of low back pain care: time for a new approach? Disabil Rehabil. 2020:1-15. 
28. Kregel J, Meeus M, Malfliet A, Dolphens M, Danneels L, Nijs J, et al. Structural and functional brain abnormalities in chronic low back pain: A systematic review. Semin Arthritis Rheum. 2015;45(2):229-37.

29. Melzack R. Pain and the neuromatrix in the brain. J Dent Educ. 2001;65(12):1378-82.

30. Aroke EN, Overstreet DS, Penn TM, Crossman DK, Jackson P, Tollefsbol TO, et al. Identification of DNA methylation associated enrichment pathways in adults with non-specific chronic low back pain. Mol Pain. 2020;16:1-17.

31. Karran EL, McAuley JH, Traeger AC, Hillier SL, Grabherr L, Russek $\mathrm{LN}$, et al. Can screening instruments accurately determine poor outcome risk in adults with recent onset low back pain? A systematic review and meta-analysis. BMC Med. 2017;15(1):13.

32. Pauli J, Starkweather A, Robins JL. Screening Tools to Predict the Development of Chronic Low Back Pain: An Integrative Review of the Literature. Pain Med. 2019;20(9):1651-77.

33. Hill JC, Dunn KM, Lewis M, Mullis R, Main CJ, Foster NE, et al. A primary care back pain screening tool: identifying patient subgroups for initial treatment. Arthritis Rheum. 2008;59(5):632-41.

34. Linton SJ, Halldén K. Can we screen for problematic back pain? A screening questionnaire for predicting outcome in acute and subacute back pain. Clin J Pain. 1998;14(3):209-15.

35. O'Connell NE, Cook CE, Wand BM, Ward SP. Clinical guidelines for low back pain: A critical review of consensus and inconsistencies across three major guidelines. Best Pract Res Clin Rheumatol. 2016;30(6):968-80.

36. Graham R. Clinical practice guidelines we can trust: National Academies Press; 2011.

37. Kredo T, Bernhardsson S, Machingaidze S, Young T, Louw Q, Ochodo $\mathrm{E}$, et al. Guide to clinical practice guidelines: the current state of play. Int J Qual Health Care. 2016;28(1):122-8.

38. National Clinical Guideline Center (NICE). Low back pain and sciatica in over 16s: assessment and management [Internet]. National Clinical Guideline Center; 2016 [uppdated 2020-12-11, cited 2021-09-03]. Avaliable from: http://www.nice.org.uk/guidance/gidcgwaveo681/documents.

39. Foster NE, Hill JC, O'Sullivan P, Hancock M. Stratified models of care. Best Pract Res Clin Rheumatol. 2013;27(5):649-61.

40. Hill JC, Whitehurst DG, Lewis M, Bryan S, Dunn KM, Foster NE, et al. Comparison of stratified primary care management for low back pain with current best practice (STarT Back): a randomised controlled trial. Lancet. 2011;378(9802):1560-71.

41. Ogbeivor C, Elsabbagh L. Management approach combining prognostic screening and targeted treatment for patients with low back pain compared with standard physiotherapy: A systematic review \& meta-analysis. Musculoskeletal Care. 2021: 1-21.

42. Kongsted A, Kent P, Quicke JG, Skou ST, Hill JC. Risk-stratified and stepped models of care for back pain and osteoarthritis: are we heading towards a common model? Pain Rep. 2020;5(5):e843.

43. Corp N, Mansell G, Stynes S, Wynne-Jones G, Morsø L, Hill JC, et al. Evidence-based treatment recommendations for neck and low back 
pain across Europe: A systematic review of guidelines. Eur J Pain. 2021;25:275-95.

44. Castellini G, Iannicelli V, Briguglio M, Corbetta D, Sconfienza LM, Banfi G, et al. Are clinical practice guidelines for low back pain interventions of high quality and updated? A systematic review using the AGREE II instrument. BMC Health Serv Res. 2020;20(1):970.

45. Fischer F, Lange K, Klose K, Greiner W, Kraemer A. Barriers and Strategies in Guideline Implementation-A Scoping Review. Healthcare (Basel). 2016;4(36).

46. Correa VC, Lugo-Agudelo LH, Aguirre-Acevedo DC, Contreras JAP, Borrero AMP, Patiño-Lugo DF, et al. Individual, health system, and contextual barriers and facilitators for the implementation of clinical practice guidelines: a systematic metareview. Health Res Policy Syst. 2020;18(1):74.

47. Fritz J Msc RPT, Söderbäck M PhD RN, Söderlund A PhD RPT, Sandborgh M PhD RPT. The complexity of integrating a behavioral medicine approach into physiotherapy clinical practice. Physiother Theory Pract. 2019;35(12):1182-93.

48. Slade SC, Kent P, Patel S, Bucknall T, Buchbinder R. Barriers to Primary Care Clinician Adherence to Clinical Guidelines for the Management of Low Back Pain: A Systematic Review and Metasynthesis of Qualitative Studies. Clin J Pain. 2016;32(9):800-16.

49. Glasgow RE, Vinson C, Chambers D, Khoury MJ, Kaplan RM, Hunter C. National Institutes of Health approaches to dissemination and implementation science: current and future directions. Am J Public Health. 2012;102(7):1274-81.

50. Moore GF, Audrey S, Barker M, Bond L, Bonell C, Hardeman W, et al. Process evaluation of complex interventions: Medical Research Council guidance. BMJ. 2015;350:h1258.

51. Berube ME, Poitras S, Bastien M, Laliberte LA, Lacharite A, Gross DP. Strategies to translate knowledge related to common musculoskeletal conditions into physiotherapy practice: a systematic review.

Physiotherapy. 2018;104(1):1-8.

52. World Health Organization, Adherence to long-term therapies : evidence for action / [edited by Eduardo Sabaté] [Internet]. World Health Organization; 2003 [uppdated 2021-03-09, cited 2021-09-03]. Avaliable from https://apps.who.int/iris/handle/10665/42682.

53. Hall A, Richmond H, Copsey B, Hansen Z, Williamson E, Jones G, et al. Physiotherapist-delivered cognitive-behavioural interventions are effective for low back pain, but can they be replicated in clinical practice? A systematic review. Disabil Rehabil. 2018;40(1):1-9.

54. Darlow B, Fullen BM, Dean S, Hurley DA, Baxter GD, Dowell A. The association between health care professional attitudes and beliefs and the attitudes and beliefs, clinical management, and outcomes of patients with low back pain: a systematic review. Eur J Pain. 2012;16(1):3-17.

55. Gardner T, Refshauge K, Smith L, McAuley J, Hubscher M, Goodall S. Physiotherapists' beliefs and attitudes influence clinical practice in chronic low back pain: a systematic review of quantitative and qualitative studies. J Physiother. 2017;63(3):132-43. 
56. Zadro J, O'Keeffe M, Maher C. Do physical therapists follow evidencebased guidelines when managing musculoskeletal conditions?

Systematic review. BMJ Open. 2019;9(10):e032329.

57. Bernhardsson S, Larsson ME, Eggertsen R, Olsen MF, Johansson K, Nilsen P, et al. Evaluation of a tailored, multi-component intervention for implementation of evidence-based clinical practice guidelines in primary care physical therapy: a non-randomized controlled trial. BMC Health Serv Res. 2014;14:105.

58. Fritz J, Wallin L, Söderlund A, Almqvist L, Sandborgh M. Implementation of a behavioral medicine approach in physiotherapy: impact and sustainability. Disabil Rehabil. 2019:1-8.

59. Fritz J. Implementation of a behavioural medicine approach in physiotherapy: Determinants, clinical behaviours, patient outcomes and the implementation process [Internet] [PhD dissertation]. [Västerås]. Mälardalen University Press Dissertations: Mälardalen University; 2020.

6o. Lindbäck Y, Enthoven P, Öberg B. Patients' experiences of how symptoms are explained and influences on back-related health after pre-surgery physiotherapy: A qualitative study. Musculoskelet Sci Pract. 2019;40:34-9.

61. Rogers EM. Diffusion of innovations.: New York: Free Press; 2003.

62. Greenhalgh T, Robert G, Macfarlane F, Bate P, Kyriakidou O. Diffusion of innovations in service organizations: systematic review and recommendations. Milbank Q. 2004;82(4):581-629.

63. Shelton RC, Cooper BR, Stirman SW. The Sustainability of EvidenceBased Interventions and Practices in Public Health and Health Care. Annual review of public health. 2018;39:55-76.

64. Curran GM, Bauer M, Mittman B, Pyne JM, Stetler C. Effectivenessimplementation hybrid designs: combining elements of clinical effectiveness and implementation research to enhance public health impact. Med Care. 2012;50(3):217-26.

65. Allotey P, Reidpath DD, Ghalib H, Pagnoni F, Skelly WC. Efficacious, effective, and embedded interventions: implementation research in infectious disease control. BMC Public Health. 2008;8:343.

66. Michie S, Johnston M. Changing clinical behaviour by making guidelines specific. BMJ. 2004;328(7435):343-5.

67. Michie S, van Stralen MM, West R. The behaviour change wheel: a new method for characterising and designing behaviour change interventions. Implement Sci. 2011;6:42.

68. Cane J, O'Connor D, Michie S. Validation of the theoretical domains framework for use in behaviour change and implementation research. Implement Sci. 2012;7:37.

69. Michie S, Johnston M, Abraham C, Lawton R, Parker D, Walker A. Making psychological theory useful for implementing evidence based practice: a consensus approach. Qual Saf Health Care. 2005;14(1):2633 .

70. Atkins L, Francis J, Islam R, O’Connor D, Patey A, Ivers N, et al. A guide to using the Theoretical Domains Framework of behaviour change to investigate implementation problems. Implementation Science. 2017;12(1):77. 
71. Lipworth W, Taylor N, Braithwaite J. Can the theoretical domains framework account for the implementation of clinical quality interventions? BMC Health Serv Res. 2013;13:530.

72. Michie S AL, West R. The behaviour Change Wheel A Guide to Designing Interventions. ed., editor. Great Britain: Silverback Publishing; 2014.

73. Michie S, Wood CE, Johnston M, Abraham C, Francis JJ, Hardeman W. Behaviour change techniques: the development and evaluation of a taxonomic method for reporting and describing behaviour change interventions (a suite of five studies involving consensus methods, randomised controlled trials and analysis of qualitative data). Health Technol Assess. 2015;19(99):1-188.

74. Isenor JE, Bai I, Cormier R, Helwig M, Reeve E, Whelan AM, et al. Deprescribing interventions in primary health care mapped to the Behaviour Change Wheel: A scoping review. Res Social Adm Pharm. 2021;17(7):1229-41.

75. Briggs AM, Jordan JE, Jennings M, Speerin R, Chua J, Bragge P, et al. A framework to evaluate musculoskeletal models of care. Cornwall: Global Alliance for Musculoskeletal Health of the Bone and Joint Decade. [Internet]. 2016 [cited 2021-09-03]. Avaliable from: https://www.aci.health.nsw.gov.au/_data/assets/pdf_file/oo20/338 141/Framework-to-Evaluate-Musculoskeletal-MoC.pdf.

76. Abbott A, Schroder K, Enthoven P, Nilsen P, Oberg B. Effectiveness of implementing a best practice primary healthcare model for low back pain (BetterBack) compared with current routine care in the Swedish context: an internal pilot study informed protocol for an effectivenessimplementation hybrid type 2 trial. BMJ Open. 2018;8(4):e019906.

77. Briggs AM, Chan M, Slater H. Models of Care for musculoskeletal health: Moving towards meaningful implementation and evaluation across conditions and care settings. Best Pract Res Clin Rheumatol. 2016;30(3):359-74.

78. Nilsen P. Implementering av evidensbaserad praktik: Gleerup; 2014.

79. Liang L, Bernhardsson S, Vernooij RW, Armstrong MJ, Bussières A, Brouwers MC, et al. Use of theory to plan or evaluate guideline implementation among physicians: a scoping review. Implement Sci. 2017;12(1):26.

8o. Tabak RG, Khoong EC, Chambers DA, Brownson RC. Bridging research and practice: models for dissemination and implementation research. Am J Prev Med. 2012;43(3):337-50.

81. Prihodova L, Guerin S, Tunney C, Kernohan WG. Key components of knowledge transfer and exchange in health services research: Findings from a systematic scoping review. J Adv Nurs. 2019;75(2):313-26.

82. Proctor EK, Powell BJ, McMillen JC. Implementation strategies: recommendations for specifying and reporting. Implement Sci. 2013;8:139.

83. Mesner SA, Foster NE, French SD. Implementation interventions to improve the management of non-specific low back pain: a systematic review. BMC Musculoskelet Disord. 2016;17:258.

84. Proctor E, Silmere H, Raghavan R, Hovmand P, Aarons G, Bunger A, et al. Outcomes for implementation research: conceptual distinctions, 
measurement challenges, and research agenda. Adm Policy Ment Health. 2011;38(2):65-76.

85. Smith JD, Hasan M. Quantitative approaches for the evaluation of implementation research studies. Psychiatry Res. 2020;283:112521.

86. Gaglio B, Shoup JA, Glasgow RE. The RE-AIM framework: a systematic review of use over time. Am J Public Health. 2013;103(6):e38-46.

87. Eldh AC, Almost J, DeCorby-Watson K, Gifford W, Harvey G, Hasson $\mathrm{H}$, et al. Clinical interventions, implementation interventions, and the potential greyness in between -a discussion paper. BMC Health Serv Res. 2017;17(1):16.

88. Wollersheim H, Hermens R, Hulscher M, Braspenning J, Ouwens M, Schouten J, et al. Clinical indicators: development and applications. Neth J Med. 2007;65(1):15-22.

89. Willmeroth T, Wesselborg B, Kuske S. Implementation Outcomes and Indicators as a New Challenge in Health Services Research: A Systematic Scoping Review. Inquiry. 2019;56:1-17.

90. Prinsen CAC, Mokkink LB, Bouter LM, Alonso J, Patrick DL, de Vet HCW, et al. COSMIN guideline for systematic reviews of patientreported outcome measures. Quality of Life Research. 2018;27(5):1147-57.

91. Mokkink LB, Terwee CB, Patrick DL, Alonso J, Stratford PW, Knol DL, et al. The COSMIN study reached international consensus on taxonomy, terminology, and definitions of measurement properties for health-related patient-reported outcomes. J Clin Epidemiol. 2010;63(7):737-45.

92. Hoy D, Brooks P, Blyth F, Buchbinder R. The Epidemiology of low back pain. Best Pract Res Clin Rheumatol. 2010;24(6):769-81.

93. Fixsen D. L. NSF, Blase K. A., Friedman R. M., Wallace F. Implementation Research: A Synthesis of the Literature.: University of South Florida; 2005.

94. Bush PL, Pluye P, Loignon C, Granikov V, Wright MT, Pelletier J-F, et al. Organizational participatory research: a systematic mixed studies review exposing its extra benefits and the key factors associated with them. Implementation Science. 2017;12(1):119.

95. Sundhedsstyrelsen. The national clinical guideline for non-surgical treatment of recently lumbar nervous system impact (lumbar radiculopathy) provides recommendations on non-surgical treatment options. [Internet]. Sundhedsstyrelsen; 2016 [cited 2021-09-03]. Avaliable from:

http://sundhedsstyrelsen.dk/da/udgivelser/2016/lumbalnerverodspaavirkning-ikke-kirurgisk-behandling.

96. Sundhedsstyrelsen. The national clinical guidelines for non-surgical treatment of newly occurring lower back pain. [Internet].

Sundhedsstyrelsen; 2016 [cited 2021-09-03]. Avaliable from: http://sundhedsstyrelsen.dk/da/udgivelser/2016/lumbalnerverodspaavirkning-ikke-kirurgisk-behandling.

97. Socialstyrelsen. Nationella riktlinjer - Metodbeskrivning. [Internet]. Socialstyrelsen; 2016 [cited 2021-09-03]. Avaliable from: https://www.socialstyrelsen.se/globalassets/sharepoint- 
dokument/dokument-webb/nationella-riktlinjer/nationella-riktlinjermetodbeskrivning.pdf

98. Atkins D, Best D, Briss PA, Eccles M, Falck-Ytter Y, Flottorp S, et al. Grading quality of evidence and strength of recommendations. BMJ. 2004;328:1490-4.

99. Leventhal H, Phillips LA, Burns E. The Common-Sense Model of SelfRegulation (CSM): a dynamic framework for understanding illness self-management. J Behav Med. 2016;39(6):935-46.

100. Smucker DR, Konrad TR, Curtis P, Carey TS. Practitioner selfconfidence and patient outcomes in acute low back pain. Arch Fam Med. 1998;7(3):223-8.

101. Bishop A. Pain Attitudes and Beliefs Scale (PABS). J Physiother. 2010;56(4):279.

102. Mutsaers JH, Peters R, Pool-Goudzwaard AL, Koes BW, Verhagen AP. Psychometric properties of the Pain Attitudes and Beliefs Scale for Physiotherapists: a systematic review. Man Ther. 2012;17(3):213-8.

103. Huijg JM, Gebhardt WA, Dusseldorp E, Verheijden MW, van der Zouwe N, Middelkoop BJ, et al. Measuring determinants of implementation behavior: psychometric properties of a questionnaire based on the theoretical domains framework. Implement Sci. 2014;9:33.

104. Huijg JM, Gebhardt WA, Crone MR, Dusseldorp E, Presseau J. Discriminant content validity of a theoretical domains framework questionnaire for use in implementation research. Implement Sci. 2014;9:11.

105. Cieza A, Stucki G, Weigl M, Disler P, Jackel W, van der Linden S, et al. ICF Core Sets for low back pain. J Rehabil Med. 2004(44 Suppl):6974 .

106. Fairbank JC, Pynsent PB. The Oswestry Disability Index. Spine (Phila Pa 1976). 2000;25(22):2940-52; discussion 52.

107. Garg A, Pathak H, Churyukanov MV, Uppin RB, Slobodin TM. Low back pain: critical assessment of various scales. Eur Spine J. 2020;29(3):503-18.

108. Clement RC, Welander A, Stowell C, Cha TD, Chen JL, Davies M, et al. A proposed set of metrics for standardized outcome reporting in the management of low back pain. Acta Orthop. 2015;86(5):523-33.

109. Ferreira-Valente MA, Pais-Ribeiro JL, Jensen MP. Validity of four pain intensity rating scales. Pain. 2011;152(10):2399-404.

110. EuroQol--a new facility for the measurement of health-related quality of life. Health Policy. 1990;16(3):199-208.

111. Chapman JR, Norvell DC, Hermsmeyer JT, Bransford RJ, DeVine J, McGirt MJ, et al. Evaluating common outcomes for measuring treatment success for chronic low back pain. Spine (Phila Pa 1976). 2011;36(21 Suppl):S54-68.

112. Broadbent E, Petrie KJ, Main J, Weinman J. The brief illness perception questionnaire. J Psychosom Res. 2006;60(6):631-7.

113. Broadbent E, Wilkes C, Koschwanez H, Weinman J, Norton S, Petrie KJ. A systematic review and meta-analysis of the Brief Illness Perception Questionnaire. Psychol Health. 2015;30(11):1361-85.

114. Howie JG, Heaney DJ, Maxwell M, Walker JJ. A comparison of a Patient Enablement Instrument (PEI) against two established 
satisfaction scales as an outcome measure of primary care consultations. Fam Pract. 1998;15(2):165-71.

115. Roost M, Zielinski A, Petersson C, Strandberg EL. Reliability and applicability of the Patient Enablement Instrument (PEI) in a Swedish general practice setting. BMC Fam Pract. 2015;16:31.

116. Lis A. Reliability and validity of a treatment outcome satisfaction questionnaire for patients with low back pain (LBP). Dissertation: New York University; 2010.

117. Lo Martire R, Lis A, Skillgate E, Rasmussen-Barr E. Psychometric properties of the Swedish version of the Treatment Outcome Satisfaction Questionnaire. Eur Spine J. 2017;26(2):316-23.

118. Kamper SJ, Maher CG, Mackay G. Global rating of change scales: a review of strengths and weaknesses and considerations for design. J Man Manip Ther. 2009;17(3):163-70.

119. Preston CC, Colman AM. Optimal number of response categories in rating scales: reliability, validity, discriminating power, and respondent preferences. Acta Psychol (Amst). 2000;104(1):1-15.

120. Hoffmann TC, Glasziou PP, Boutron I, Milne R, Perera R, Moher D, et al. Better reporting of interventions: template for intervention description and replication (TIDieR) checklist and guide. BMJ. 2014;348:g1687.

121. Beaton DE, Bombardier C, Guillemin F, Ferraz MB. Guidelines for the process of cross-cultural adaptation of self-report measures. Spine (Phila Pa 1976). 2000;25(24):3186-91.

122. Epstein J, Santo RM, Guillemin F. A review of guidelines for crosscultural adaptation of questionnaires could not bring out a consensus. J Clin Epidemiol. 2015;68(4):435-41.

123. Beckstead JW. Content validity is naught. Int J Nurs Stud. 2009;46(9):1274-83.

124. Brown T. Confirmatory Factor Analysis for Applied Research: The Gillford Press; 2006.

125. Perry JL, Nicholls AR, Clough PJ, Crust L. Assessing Model Fit: Caveats and Recommendations for Confirmatory Factor Analysis and Exploratory Structural Equation Modeling. Measurement in Physical Education \& Exercise Science. 2015;19(1):12-21.

126. Lakens D. Calculating and reporting effect sizes to facilitate cumulative science: a practical primer for t-tests and ANOVAs. Frontiers in psychology. 2013;4:863.

127. Azur MJ, Stuart EA, Frangakis C, Leaf PJ. Multiple imputation by chained equations: what is it and how does it work? International journal of methods in psychiatric research. 2011;20(1):40-9.

128. Sterne JA, White IR, Carlin JB, Spratt M, Royston P, Kenward MG, et al. Multiple imputation for missing data in epidemiological and clinical research: potential and pitfalls. BMJ 2009;338:b2393.

129. Hooper R, Bourke L. The dog-leg: an alternative to a cross-over design for pragmatic clinical trials in relatively stable populations. Int $\mathrm{J}$ Epidemiol. 2014;43(3):930-6.

130. Revicki D, Hays RD, Cella D, Sloan J. Recommended methods for determining responsiveness and minimally important differences for patient-reported outcomes. J Clin Epidemiol. 2008;61(2):102-9. 
131. van der Roer N, Ostelo RW, Bekkering GE, van Tulder MW, de Vet HC. Minimal clinically important change for pain intensity, functional status, and general health status in patients with nonspecific low back pain. Spine (Phila Pa 1976). 2006;31(5):578-82.

132. Soer R, Reneman MF, Vroomen PC, Stegeman P, Coppes MH. Responsiveness and minimal clinically important change of the Pain Disability Index in patients with chronic back pain. Spine (Phila Pa 1976). 2012;37(8):711-5.

133. Hosmer DW LS. Applied Logistic Regression. New York, USA: John Wiley and Sons; 2000.

134. Fox-Wasylyshyn SM, El-Masri MM. Handling missing data in selfreport measures. Res Nurs Health. 2005;28(6):488-95.

135. Ris I, Schröder K, Kongsted A, Abbott A, Nilsen P, Hartvigsen J, et al. Adapting the determinants of implementation behavior questionnaire to evaluate implementation of a structured low back pain programme using mixed-methods. Health Sci Rep. 2021;4(2):e266.

136. Terwee CB, Bot SD, de Boer MR, van der Windt DA, Knol DL, Dekker $\mathrm{J}$, et al. Quality criteria were proposed for measurement properties of health status questionnaires. J Clin Epidemiol. 2007;60(1):34-42.

137. Enthoven P, Eddeborn F, Abbott A, Schröder K, Fors M, Öberg B. Patients' experiences of the BetterBack model of care for low back pain in primary care - a qualitative interview study. Int J Qual Stud Health Well-being. 2021;16(1):1861719.

138. Simmonds MJ, Derghazarian T, Vlaeyen JW. Physiotherapists' knowledge, attitudes, and intolerance of uncertainty influence decision making in low back pain. Clin J Pain. 2012;28(6):467-74.

139. Jacobs CM, Guildford BJ, Travers W, Davies M, McCracken LM. Brief psychologically informed physiotherapy training is associated with changes in physiotherapists' attitudes and beliefs towards working with people with chronic pain. Br J Pain. 2016;10(1):38-45.

140. Overmeer T, Boersma K, Main CJ, Linton SJ. Do physical therapists change their beliefs, attitudes, knowledge, skills and behaviour after a biopsychosocially orientated university course? J Eval Clin Pract. 2009;15(4):724-32.

141. Synnott A, O'Keeffe M, Bunzli S, Dankaerts W, O'Sullivan P, Robinson $\mathrm{K}$, et al. Physiotherapists report improved understanding of and attitude toward the cognitive, psychological and social dimensions of chronic low back pain after Cognitive Functional Therapy training: a qualitative study. J Physiother. 2016;62(4):215-21.

142. Stander J, Grimmer K, Brink Y. Training programmes to improve evidence uptake and utilisation by physiotherapists: a systematic scoping review. BMC Medical Education. 2018;18(1):14.

143. Bekkering GE, Hendriks HJ, van Tulder MW, Knol DL, Hoeijenbos M, Oostendorp RA, et al. Effect on the process of care of an active strategy to implement clinical guidelines on physiotherapy for low back pain: a cluster randomised controlled trial. Qual Saf Health Care. 2005;14(2):107-12.

144. van der Wees PJ, Jamtvedt G, Rebbeck T, de Bie RA, Dekker J, Hendriks EJ. Multifaceted strategies may increase implementation of physiotherapy clinical guidelines: a systematic review. Aust J Physiother. 2008;54(4):233-41. 
145. van Dulmen SA, Maas M, Staal JB, Rutten G, Kiers H, Nijhuis-van der Sanden M, et al. Effectiveness of peer assessment for implementing a Dutch physical therapy low back pain guideline: cluster randomized controlled trial. Phys Ther. 2014;94(10):1396-409.

146. Evans DW, Breen AC, Pincus T, Sim J, Underwood M, Vogel S, et al. The effectiveness of a posted information package on the beliefs and behavior of musculoskeletal practitioners: the UK Chiropractors, Osteopaths, and Musculoskeletal Physiotherapists Low Back Pain ManagemENT (COMPLeMENT) randomized trial. Spine (Phila Pa 1976). 2010;35(8):858-66.

147. Rutten GM, Harting J, Bartholomew LK, Schlief A, Oostendorp RA, de Vries NK. Evaluation of the theory-based Quality Improvement in Physical Therapy (QUIP) programme: a one-group, pre-test post-test pilot study. BMC Health Serv Res. 2013;13:194.

148. Synnott A, O'Keeffe M, Bunzli S, Dankaerts W, O'Sullivan P, O'Sullivan K. Physiotherapists may stigmatise or feel unprepared to treat people with low back pain and psychosocial factors that influence recovery: a systematic review. J Physiother. 2015;61(2):68-76.

149. Swinkels IC, van den Ende CH, van den Bosch W, Dekker J, Wimmers $\mathrm{RH}$. Physiotherapy management of low back pain: does practice match the Dutch guidelines? Aust J Physiother. 2005;51(1):35-41.

150. Goodwin RW, Hendrick PA. Physiotherapy as a first point of contact in general practice: a solution to a growing problem? Prim Health Care Res Dev. 2016;17(5):489-502.

151. Chou R, Fu R, Carrino JA, Deyo RA. Imaging strategies for low-back pain: systematic review and meta-analysis. Lancet. 2009;373(9662):463-72.

152. Bernhardsson S, Larsson MEH. Does a tailored guideline implementation strategy have an impact on clinical physiotherapy practice? A nonrandomized controlled study. J Eval Clin Pract. 2018:110.

153. Carlfjord S, Nilsing-Strid E, Johansson K, Holmgren T, Öberg B. Practitioner experiences from the structured implementation of evidence-based practice in primary care physiotherapy: A qualitative study. J Eval Clin Pract. 2019;25(4):622-9.

154. Ris I, Boyle E, Myburgh C, Hartvigsen J, Thomassen L, Kongsted A. Factors influencing implementation of the GLA: D Back, an educational/exercise intervention for low back pain: a mixed-methods study. JBI Evid Implement. Forthcoming 2021.

155. Al Zoubi FM, Menon A, Mayo NE, Bussières AE. The effectiveness of interventions designed to increase the uptake of clinical practice guidelines and best practices among musculoskeletal professionals: a systematic review. BMC Health Serv Res. 2018;18(1):435.

156. Suman A, Dikkers MF, Schaafsma FG, van Tulder MW, Anema JR. Effectiveness of multifaceted implementation strategies for the implementation of back and neck pain guidelines in health care: a systematic review. Implement Sci. 2016;11(1):126.

157. Beneciuk JM, George SZ. Pragmatic Implementation of a Stratified Primary Care Model for Low Back Pain Management in Outpatient Physical Therapy Settings: Two-Phase, Sequential Preliminary Study. Phys Ther. 2015;95(8):1120-34. 
158. Cherkin D, Balderson B, Wellman R, Hsu C, Sherman KJ, Evers SC, et al. Effect of Low Back Pain Risk-Stratification Strategy on Patient Outcomes and Care Processes: the MATCH Randomized Trial in Primary Care. J Gen Intern Med. 2018;33(8):1324-36.

159. Foster NE. Barriers and progress in the treatment of low back pain. BMC Med. 2011;9:108.

160. Bamm EL, Rosenbaum P, Wilkins S. Is Health Related Quality Of Life of people living with chronic conditions related to patient satisfaction with care? Disabil Rehabil. 2013;35(9):766-74.

161. Fritz JM, Cleland JA, Speckman M, Brennan GP, Hunter SJ. Physical therapy for acute low back pain: associations with subsequent healthcare costs. Spine (Phila Pa 1976). 2008;33(16):1800-5.

162. Ostelo RW, Deyo RA, Stratford P, Waddell G, Croft P, Von Korff M, et al. Interpreting change scores for pain and functional status in low back pain: towards international consensus regarding minimal important change. Spine (Phila Pa 1976). 2008;33(1):90-4.

163. Landes SJ, McBain SA, Curran GM. An introduction to effectivenessimplementation hybrid designs. Psychiatry Res. 2019;280:112513.

164. Gagne P, Hancock GR. Measurement model quality, sample size, and solution propriety in confirmatory factor models. Multivariate Behavioral Research. 2006;41(1):65-83.

165. Maas MJ, van der Wees PJ, Braam C, Koetsenruijter J, Heerkens YF, van der Vleuten $\mathrm{CP}$, et al. An innovative peer assessment approach to enhance guideline adherence in physical therapy: single-masked, cluster-randomized controlled trial. Phys Ther. 2015;95(4):600-12.

166. Rutten G, Kremers S, Rutten S, Harting J. A theory-based crosssectional survey demonstrated the important role of awareness in guideline implementation. J Clin Epidemiol. 2009;62(2):167-76.

167. Chiarotto A, Terwee CB, Ostelo RW. Choosing the right outcome measurement instruments for patients with low back pain. Best Pract Res Clin Rheumatol. 2016;30(6):1003-20.

168. Chiarotto A, Boers M, Deyo RA, Buchbinder R, Corbin TP, Costa LOP, et al. Core outcome measurement instruments for clinical trials in nonspecific low back pain. Pain. 2018;159(3):481-95.

169. Jette DU, Bacon K, Batty C, Carlson M, Ferland A, Hemingway RD, et al. Evidence-based practice: beliefs, attitudes, knowledge, and behaviors of physical therapists. Phys Ther. 2003;83(9):786-805.

170. McCleary L. Using multiple imputation for analysis of incomplete data in clinical research. Nurs Res. 2002;51(5):339-43.

171. Folkhälsomyndigheten. Open comparisons public health. [Internet]. 2019. [uppdated 2019-02-08, cited 2021-09-03]. Avaliable from: https://www.folkhalsomyndigheten.se/publiceratmaterial/publikationsarkiv/oe/oppna-jamforelser-folkhalsa-2019/

172. Bier JD, Sandee-Geurts JJW, Ostelo R, Koes BW, Verhagen AP. Can Primary Care for Back and/or Neck Pain in the Netherlands Benefit From Stratification for Risk Groups According to the STarT Back Tool Classification? Arch Phys Med Rehabil. 2018;99(1):65-71.

173. Enthoven P, Skargren E, Oberg B. Clinical course in patients seeking primary care for back or neck pain: a prospective 5-year follow-up of outcome and health care consumption with subgroup analysis. Spine (Phila Pa 1976). 2004;29(21):2458-65. 
174. Nilsen P, Bernhardsson S. Context matters in implementation science: a scoping review of determinant frameworks that describe contextual determinants for implementation outcomes. BMC Health Serv Res. 2019;19(1):189. 

Appendix 1.

\section{BetterBack:- Model of care for LBP}

Östergötland health care region physiotherapeutic clinical practice guideline recommendations for primary care management of benign LBP with or without radiculopathy

Each evidence based guideline recommendation is supported by a clinical priority ranking. This is based on an overall assessment of the severity of the condition, reported effect of the intervention, strength of evidence assessment (GRADE), cost-effectiveness and the benefit of the intervention based on professional experience and patient benefit. A scale from 1 to 10 is used where the number 1 indicates recommended practices with the highest priority while the number 9 indicates recommended practices of low priority. The number 10 indicates recommendations that provide very little or no benefit or utility and are therefore not recommended.

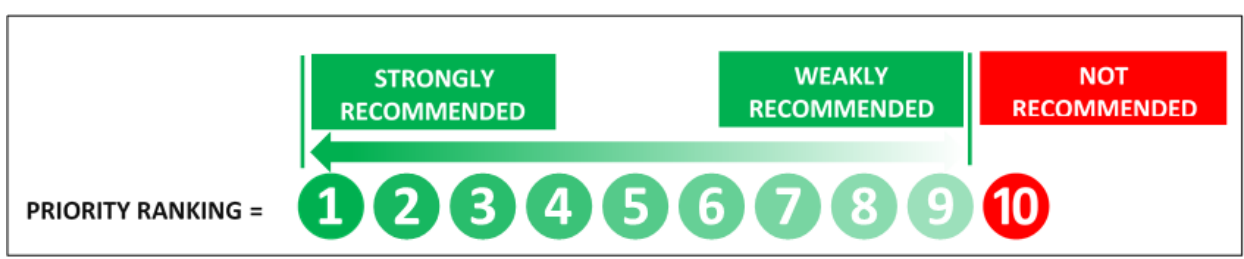

\section{Recommendation 1}

PRIORITY RANKING = 1 (2) 3 (2)

Routine care should consist of standardised processes for subjective and objective assessment and diagnostics. A thorough screening of red flags is essential to rule out serious pathology. Treatment should be individualised for each patient. Basic treatment principles should be based on reassurance of a good prognosis, maintenance of appropriate physical activity and self-care enablement.

Justification: The work group's reasoning is based on clinical experience of the importance of careful screening to rule out serious pathology. Furthermore, standardised assessment and diagnostics provide quality assurance but treatment needs to be individualised for each patient case. The work group also reasoned based on clinical experience that appropriate physical activity is likely to contribute to maintaining the patient's functional level, psychosocial and general health as well as have positive effects on self-care enablement. In some cases, may physical activity temporarily aggravate pain and symptoms, but there are no known persisting side effects. The work groups reasoning is also based on evidence showing a statistically significant advantage for maintaining appropriate physical activity compared to bed rest for improving pain and function. Despite this, evidence that proves the benefit of appropriate physical activity is so great to be clinically relevant is missing. In addition, the best available evidence has however a currently limited scientific basis $(\otimes \otimes \bigcirc \bigcirc)$. The working group proposes the following resources in the

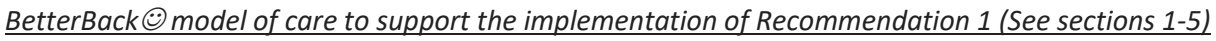




\section{Recommendation 2}

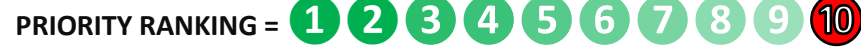

Do not perform routine medical imaging investigations (eg X-ray, CT, MRI)

Justification: The work group's reasoning is based on evidence that shows no differences in outcomes of pain, function and quality of life between patients who received or did not receive routine medical imaging investigations in the primary care context. The best available evidence has however a currently inadequate scientific basis $(\otimes \bigcirc \bigcirc)$ ). It was also discussed that imaging cannot confirm or reject a preliminary diagnosis as the relationship between patient symptoms and degenerative imaging finding is usually weak. Moreover, degenerative secondary findings are common in asymptomatic individuals. The work group however suggests that early use of medical imaging is motivated in the presence of symptoms or signs suggesting possible serious underlying pathology (red flags). Medical imaging may also be relevant when pain persists despite primary care treatment.

\section{Recommendation 3}

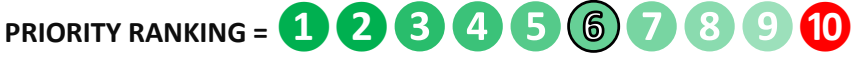

Consider using a patient-reported tool (eg STarT Back risk assessment tool) as usual care during the early-stages of patient management to screen the risk of continued LBP

Justification: The work group's reasoning is based on studies showing that STarT Back Tool is the only valid tool to investigate the risk of continued back pain in the primary care context. It shows the highest accuracy for detecting patients with low risk profile (total score $\leq 3$ ) and medium-high risk profile (total score $\geq 4$ ) for continued back pain. Studies also show that STarT Back Tool has the best ability to predict functional and pain-related outcomes. The best available evidence has however a currently inadequate scientific basis $(\otimes \bigcirc \bigcirc \bigcirc)$. No economical evaluations were identified but the working group discussed the importance of a simple and fast tool. STarT Back Tool can be filled in and analyzed in a few minutes to advantage over other tools that can be an administrative burden for patients and healthcare professionals. The working group argues that the predictive value of the tool should support, but not replace, regular examination procedures and clinical decision making. See section 3 for STarT Back Tool.

\section{Recommendation 4}

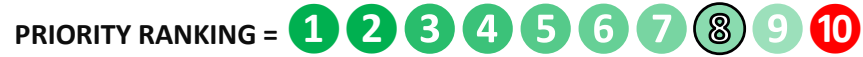

Consider using a patient-reported tool (such as the STarT Back risk assessment tool) and classification of examination findings during the early-stages of patient management to aid the stratification of care to prevent continued LBP

Justification: The work group reasoned that for the choice and scope of targeted treatment measures, consideration should be given to the assessment of risk profile for long-term LBP and classification of examination findings. This has been shown to have a better effect on pain, function and quality of life, as well as less economic costs compared to no treatment stratification. The best available evidence has however a currently inadequate scientific basis $(\otimes \bigcirc \bigcirc \bigcirc)$. For a patient with low risk profile (total score $\leq 3$ on STarT Back Tool) usual care is relevant and requires only few visits, but the working group recommends that adequate treatment measures directed at examination findings is of the highest importance. For patients with medium-high risk profile (total score $\geq 4$ on STarT Back Tool), usual care will require additional visits. Information provided in questions 5-9 on STarT Back Tool that investigate anxiety with psychological risk factors can guide the need, focus and extent of behavioral medicine measures. The working group argues that stratified care classified after assessing a risk profile for long-term back pain should support but not replace conventional examination procedures and clinical decision-making for treatment measures. The working group proposes the following resources to support the implementation of targeted treatments based on stratification (See sections 1-5). 


\section{Recommendation 5}

PRIORITY RANKING $=\begin{array}{llllllllll}1 & 2 & 3 & 4 & 4 & 6 & 7 & 8 & 9 & 10\end{array}$

Consider giving individualised patient education as a part of usual care (e.g. an explanatory model based on pain neuroscience and psychological mechanisms)

Justification: Based on the best available evidence, the work group reasoned that individualised patient education as part of usual care can result in reduced work sickness absenteeism. The priority of the recommendation has been strengthened by consensus within the work group based on proven experience that individual adapted patient education is an important part of patientcentered care. The best available evidence has however a currently inadequate scientific basis $(\otimes \bigcirc \bigcirc \bigcirc)$. The intervention requires that the patient is receptive for education. The extent of patient education can depend upon whether the patient has a distorted image of the underlying mechanism of LBP and a high degree of negative outcome expectations, anxiety, and fearavoidance or if they are inactive or passive in managing the LBP. Patient education should include a reassuring dialogue and other cognitive and behavioural therapeutic techniques of relevance to support change in the individual's maladaptive thoughts, feelings and behaviors. Pedagogical explanation models should be used to provide the patient with knowledge about symptoms and disorders, as well as to strengthen and support self-care ability to master everyday activities. The work group proposes the following resources to support of the implementation of patient education (See sections 6-7)

\section{Recommendation 6}

\section{PRIORITY RANKING $=\begin{array}{llllllllll}1 & 2 & 3 & 4 & 5 & 6 & 7 & 8 & 9 & 10\end{array}$}

\section{Consider a supervised exercise program as part of usual care}

Justification: Supervised training is defined as general or back-specific exercises or physical activities conducted under the guidance of a healthcare professionals. The work group's reasoning is based on scientific evidence and proven experience that supervised training as part of usual care can result in clinically relevant improvement in pain, function, quality of life and produces lower health care costs compared with no supervised training. There is however no evidence that a specific type of exercise would be superior to another. The best available evidence has however a currently limited scientific basis $(\otimes \otimes \bigcirc \bigcirc)$.

The work group proposes the following resources to support the implementation of a supervised training program (see section 8).

\section{Recommendation 7}

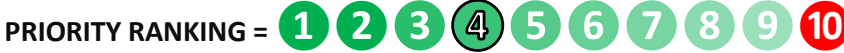

Consider mobilisation techniques for neuromusculoskeletal structures as part of usual care (including active or passive motion in an angular and / or translational plane)

Justification: The working group reasoning is based on evidence that for patients with segmental movement impairments, mobilization techniques can provide a statistically significant reduction in short-term pain. It is however uncertain whether the effect is sufficiently large so that patients experience a clear improvement overtime. At group level, there is no evidence that a particular technique is be superior to another. It cannot be ruled out that for subgroups of LBP patients, more positive effects on pain and function may be produced by specific mobilisation techniques. It is expected that these subgroups can be identified by careful diagnostics and short trial treatments. Mobilizing techniques as part of multimodal treatment provide better results. Serious side effects are rare. However, the best available evidence is based on a currently limited scientific basis $(\otimes \otimes \bigcirc \bigcirc)$. 


\section{Recommendation 8}

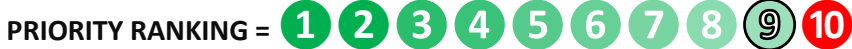

\section{Consider acupuncture treatment in addition to usual care}

Justification: The working group reasoned based on evidence that cannot exclude acupuncture has a short-term pain relief effect in addition to a placebo effect. Acupuncture has however no effect on function. Side effects in the form of brief superficial bleeding or inflammation may occur. Pneumothorax and systemic infections are not common, but the prevalence is unknown. The best available evidence has however a currently inadequate scientific basis $(\otimes 0 \bigcirc \bigcirc)$.

\section{Recommendation 9}

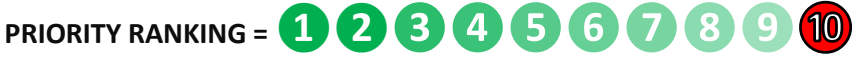

\section{Do not offer corset, shoes, traction, ultrasound or electrotherapy}

Justification: The work group's reasoning is based on evidence that passive treatments such as corset, shoots / soles, traction, ultrasound or electrotherapy do not reduce pain or improve function and quality of life in patients more than no treatment or when offered as part of multimodal treatment. However, the best available evidence is based on a currently limited scientific basis $(\otimes \otimes \bigcirc \bigcirc)$. It cannot be ruled out that subgroups of patients may experience positive effects of these interventions when a hypothesised effect mechanism is aimed at specific functional impairment or activity limitation.

\section{Recommendation 10}

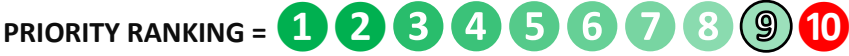

Consider prescription-free NSAID medication if necessary in addition to usual treatment (lowest dose and shortest possible treatment time).

NSAIDs: There is evidence of the effect of NSAID in patients with long-term LBP but the effect has not been highlighted on short-term pain or functional outcomes. There are no adverse reactions reported in systematic review studies on LBP, but potential transient side effects of NSAIDs such as reduced blood clotting, reduced stomach mucous function and reduced kidney function are known from studies on other conditions. The work group reasoned that lowest dose and shortest possible treatment time decreases the risk of side-effects. The work group anticipates that there are differences in patient preferences regarding NSAIDs, where some patients will agree to NSAID treatment, while others will decline. The best available evidence for NSAID effects on LBP outcomes is based on an inadequate scientific evidence ( $\bigotimes \bigcirc \bigcirc \bigcirc)$. The work group reasoned based on clinical experience that it cannot be excluded that the NSAID may have a pain relief effect in the short term.

\section{Recommendation 11}

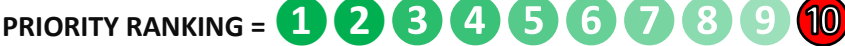

\section{Do not offer paracetamol or opioids}

Paracetomol: Has no effect on the degree of LBP and functional ability. There are no reported adverse reactions in studies, but side effects of paracetamol in the form of hepatic effects are known from studies on other conditions. The best available evidence is based on a moderately strong scientific basis $(\otimes \otimes \otimes \bigcirc)$.

Opioids: A weak analgesic effect of oxycodone in combination with paracetamol has been demonstrated in a study but the intervention has no effect on functional capacity for up to 12 weeks. Other positive effects or adverse effects were not shown. A wide range of opioid side effects are known from other studies. Therefore, the working group reasoned that treatment results in more risks than benefits to the patient. The best available evidence is based on a currently limited scientific basis $(\otimes \otimes \bigcirc \bigcirc)$. 
Appendix 1.

BetterBack:-) model of care implementation support tools

1. Subjective assessment proformer for therapist use

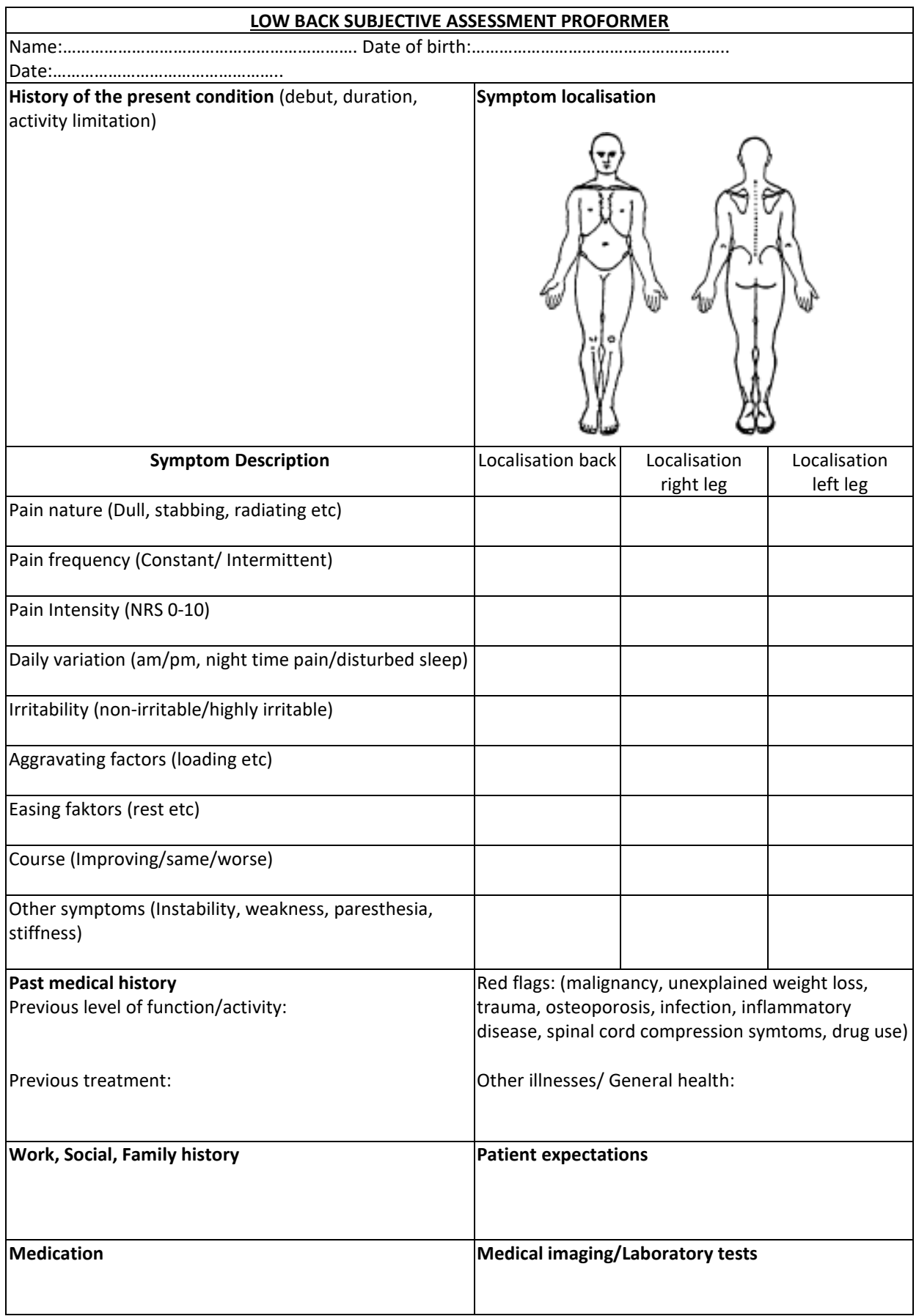


Appendix 1.

2. Physical assessment proformer

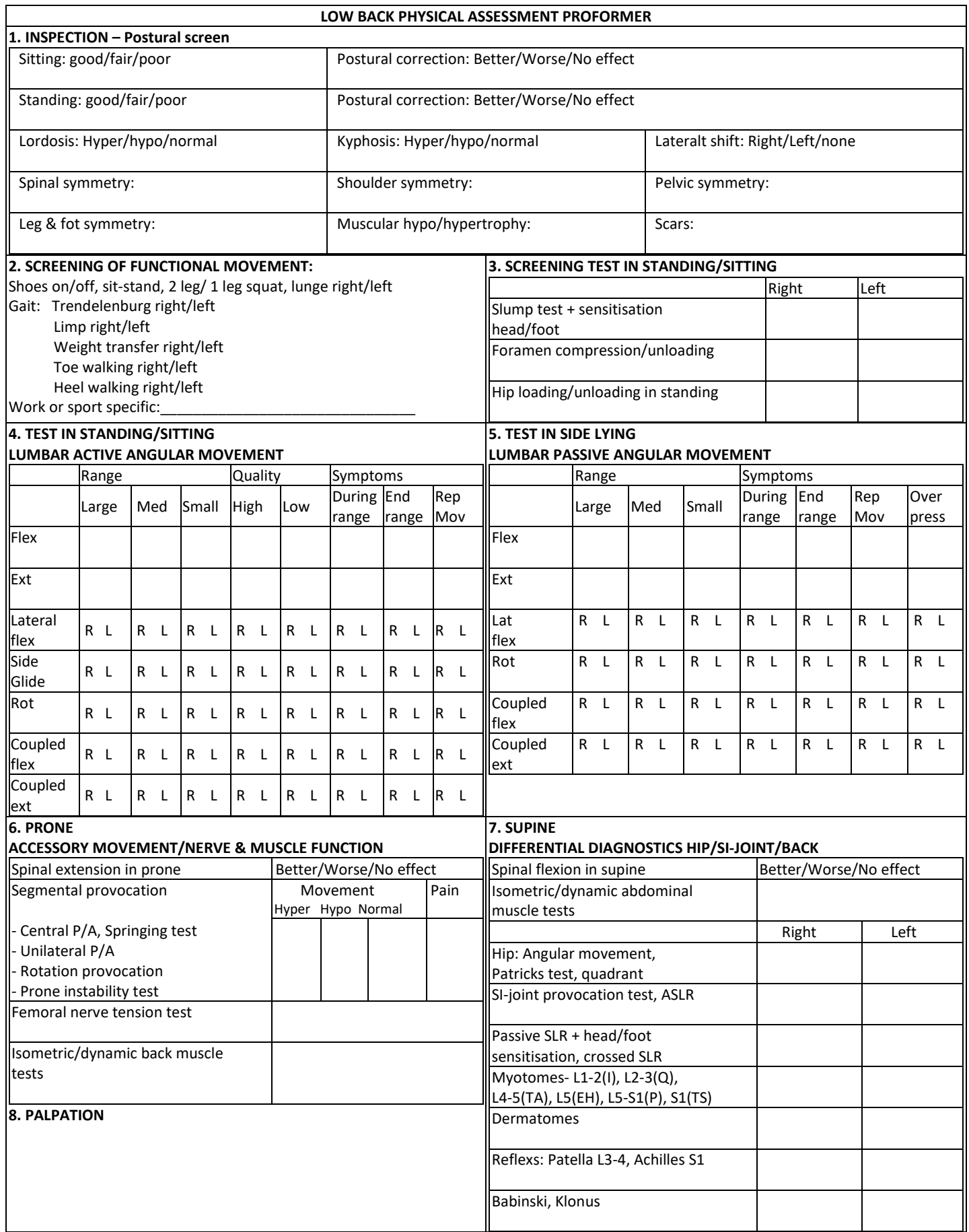


Appendix 1.

3. STarT Back Tool

Patient name:

Date:

Thinking about the last 2 weeks tick your response to the following questions:

\begin{tabular}{llll} 
& & Disagree & Agree \\
\cline { 2 - 4 } 1 & My back pain has spread down my leg(s) at some time in the last 2 weeks & $\square$ & $\square$ \\
\hline 2 & I have had pain in the shoulder or neck at some time in the last 2 weeks & $\square$ & $\square$ \\
\hline 3 & I have only walked short distances because of my back pain & $\square$ & $\square$ \\
\hline $4 \quad$ In the last 2 weeks, I have dressed more slowly than usual because of back pain & $\square$ & $\square$ \\
\hline 5 & It's not really safe for a person with a condition like mine to be physically active & $\square$ & $\square$ \\
\hline 6 & Worrying thoughts have been going through my mind a lot of the time & $\square$ & $\square$ \\
\hline 7 & I feel that my back pain is terrible and it's never going to get any better & $\square$ & $\square$ \\
\hline 8 & In general I have not enjoyed all the things I used to enjoy & $\square$ & $\square$ \\
\hline
\end{tabular}

9. Overall, how bothersome has your back pain been in the last 2 weeks?

$\begin{array}{ccccc}\text { Not at all } & \text { Slightly } & \text { Moderately } & \text { Very much } & \text { Extremely } \\ \square & \square & \square & \square & \square \\ 0 & 0 & 0 & 1 & 1\end{array}$

Total score (all 9):

Sub Score (Q5-9):

Keele University $01 / 08 / 07$

Funded by Arthritis Research UK 
Appendix 1.

4. Clinical Reasoning and Process Evaluation tool (CRPE-tool) for therapists

PATIENT NAME: DATE OF BIRTH:
First assessment date:

Final assessment date:

Totalt number of physiotherapy visits:

\section{ASSESSMENT}

- $\quad$ First assessment - cross $X$ relevant assessment findings

- Final assessment - circle $\bigcirc$ relevant assessment findings

\section{Assess grade of FUNCTIONAL IMPAIRMENT}

Energy and drive (motivation)

Sleep functions

Emotional functions (anxiety, low mood)

Thought functions (physical symptoms caused by

cognitive/rational factors)

Sensory function (sensitivity for pain "sensitisation")

Pain (choose relevant category)

\section{\begin{tabular}{l} 
Back pain \\
\hline Lower extremity pain \\
\hline Pain in a dermatome
\end{tabular} \\ Pain in a dermatome}

Pain in another body part (Buttock, hip, groin, thigh)

Generalised pain localisation (3 of 4 body quadrats)

Exercise tolerance (endurance related activities)

\section{Joint mobility}

Joint stability

Muscle power

Muscle tone

Muscle endurance

Motor reflex funktions (decreased or increased)

Control of movement (Quality, coordination, balance)

Gait pattern

Sensation of muscle stiffness, tightness, spasm, contraction, heaviness

Mobility of spinal meningies, periferal nerves and surrounding tissue

\section{Assess grade of ACTIVITY LIMITATION}

Perception of non-harmful sensory stimuli (kinesiophobia)

Carrying out daily routine (ADL)

Handling stress and other psychological demands

Changing and maintaining body position (Shifting body weight

away from the spine (increased lever arm)

Changing and maintaining body position (bending)

Maintaining a lying position

Maintaining a sitting position

Maintaining a standing position

Maintaining an upright neutral posture

Lyfting and carrying objects

\section{Walkning}

Moving around in different ways (crawling/climbing,

running/joging, jumping)

Household tasks

Work ability and employment

Recreation and leisure activities

\begin{tabular}{|c|c|c|c|c|c|}
\hline None & Lite & Moderate & Severe & Complete & $\begin{array}{l}\text { KVÅ } \\
\text { code }\end{array}$ \\
\hline 0 & 1 & 2 & 3 & 4 & PA006 \\
\hline 0 & 1 & 2 & 3 & 4 & PA007 \\
\hline 0 & 1 & 2 & 3 & 4 & PA011 \\
\hline 0 & 1 & 2 & 3 & 4 & PA013 \\
\hline 0 & 1 & 2 & 3 & 4 & PB008 \\
\hline 0 & 1 & 2 & 3 & 4 & PB009 \\
\hline 0 & 1 & 2 & 3 & 4 & PB009 \\
\hline 0 & 1 & 2 & 3 & 4 & PB009 \\
\hline 0 & 1 & 2 & 3 & 4 & PB009 \\
\hline 0 & 1 & 2 & 3 & 4 & PB009 \\
\hline 0 & 1 & 2 & 3 & 4 & PD009 \\
\hline 0 & 1 & 2 & 3 & 4 & PG001 \\
\hline 0 & 1 & 2 & 3 & 4 & PG002 \\
\hline 0 & 1 & 2 & 3 & 4 & PG003 \\
\hline 0 & 1 & 2 & 3 & 4 & PG003 \\
\hline 0 & 1 & 2 & 3 & 4 & PG003 \\
\hline 0 & 1 & 2 & 3 & 4 & PG004 \\
\hline 0 & 1 & 2 & 3 & 4 & PG006 \\
\hline 0 & 1 & 2 & 3 & 4 & PG007 \\
\hline 0 & 1 & 2 & 3 & 4 & PG003 \\
\hline 0 & 1 & 2 & 3 & 4 & PG000 \\
\hline None & Lite & Moderate & Severe & Complete & $\begin{array}{l}\text { KVÅ } \\
\text { code }\end{array}$ \\
\hline 0 & 1 & 2 & 3 & 4 & PJ001 \\
\hline 0 & 1 & 2 & 3 & 4 & PK003 \\
\hline 0 & 1 & 2 & 3 & 4 & PK004 \\
\hline 0 & 1 & 2 & 3 & 4 & PM001 \\
\hline 0 & 1 & 2 & 3 & 4 & PM001 \\
\hline 0 & 1 & 2 & 3 & 4 & PM001 \\
\hline 0 & 1 & 2 & 3 & 4 & PM001 \\
\hline 0 & 1 & 2 & 3 & 4 & PM001 \\
\hline 0 & 1 & 2 & 3 & 4 & PM001 \\
\hline 0 & 1 & 2 & 3 & 4 & PM004 \\
\hline 0 & 1 & 2 & 3 & 4 & PM007 \\
\hline 0 & 1 & 2 & 3 & 4 & PM008 \\
\hline 0 & 1 & 2 & 3 & 4 & PP003 \\
\hline 0 & 1 & 2 & 3 & 4 & PR002 \\
\hline 0 & 1 & 2 & 3 & 4 & PSO02 \\
\hline
\end{tabular}




\section{DIAGNOSTIC SUBGROUPING AND ICD-10 CODING}

\section{Matching assessment findings to diagnostic codes}

Choose a primary assessment finding category:

- First assessment: Cross $X$ one or more related ICD-10 diagnostic codes in the same row

- Final assessment: Circle $\bigcirc$ a new diagnostic codes if relevant.

\begin{tabular}{l}
\hline Primary assessment category \\
\hline LBP with muscular functional impairment \\
LBP with segmental mobility impairment \\
LBP with movement coordination impairment/ segmental \\
instability \\
$\begin{array}{l}\text { LBP with referred lower extremity pain (nociceptive pain } \\
\text { proximal of the knee) }\end{array}$
\end{tabular}

proximal of the knee)

\section{ICD-10 diagnos}

M54.5 Lumbago

M54.5 Lumbago

M99.0 Segmental dysfunction

M54.5 Lumbago

M99.1K Segmental instability in the lumbar spine

M54.5 Lumbago

$\square$ M51.2 Other specificed dislocation of intervertebral disc

M47.9K Spondylosis in the lumbar spine

LBP with radiating pain (neuropathic pain)

M54.5 Lumbago

M54.1 Radiculopathy (femoralis)

M54.4 Lumbago with ischias

LBP with related cognitive or affective tendensies

M54.5 Lumbago

$\square$ G96.8 Other specified disorders of the CNS (pain sensitivity)

LBP with related generaliserad pain (pain in 3 of 4 body quadrants)

$\square$ M54.5 Lumbago

$\square$ G96.8 Other specified disorders of the CNS (pain sensitivity)

F45.4 Chronic somatoform pain syndrome

LBP with postural related symptoms

M54.5 Lumbago

M40.3 Flatback syndrome

M40.4 Hyperlodosis

SI-joint symptoms or Coccygodynia

LBP radiating pain + Medical imaging disc pathology and nerve compression finding

LBP with radiating pain/neurogenic claudication + Medical imaging verified degeneration and nerve compression findings

LBP with movement coordination impairment/ segmental instability + Medical imaging verified Spondylolys/Spondylolisthes
M53.3 Sacrococcygeal disorders

M51.1K Disc degeneration/disc herniation in the lumbar spine with radiculopathy

M48.0K Central spinal stenos in the lumbar spine (bilateral symptoms)

M99.6 Stenosis of intervertebral foramin (unilateralt symptoms)

M43.0 Spondylolys

M43.1 Spondylolistes 
Appendix 1.

\section{TREATMENT}

4. Record at final assessment:

Has the BetterBack-i) model of care Part 1 been applied?

Has the BetterBack $\odot$ model of care Part 2 been applied? Cross $\mathrm{X}$ all modes och types of treatments used

Physical exercis MODE

$\square$ Non-supervised individual training

$\square$ Supervised individual training

$\square$ Supervised group training

TYPE

$\square$ Muscle strengthening training

$\square$ Range of movement training

$\square$ Muscle endurance training

$\square$ Cardiovascular training

$\square$ Balance training

$\square$ Postural control training

$\square$ Coordination training

$\square$ Pelvic floor training

$\square$ Postural training

$\square$ Relaxation training

$\square$ Physical activity prescription (FaR ${ }^{\circledast}$ )

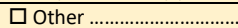

Behavioural medicine interventions MODE

\begin{tabular}{|l|l}
\hline Individual based intervention & QV011
\end{tabular}

$\square$ Group based intervention

TYPE

$\square$ Information / education on pain

QV012

\begin{tabular}{l|l} 
QV007 \\
\hline
\end{tabular}

$\square$ Cognitive-behavioural therapy

DU011

$\square$ Mindfulness

$\square$ Motivational interviewing

$\square$ Relapse prevention

$\square$ Supportive conversation

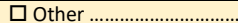

Manual therapy TYPE

$\square$ Joint mobilisation

$\square$ Joint manipulation

$\square$ Massage

$\square$ stretching

$\square$ Nerve mobiliseration

$\square$ Trigger point pressure

$\square$ Traction

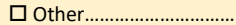

Occupational medicine interventions

TYPE

$\square$ Workplace training

$\square$ Training of work ability

$\square$ Work and employment counciling

$\square$ Information /education on ergonomics

$\square$ Other

Physical modalities

TYP

$\square$ TENS

$\square$ Cryotherapy

$\square$ Heat

$\square$ Ultrasound

$\square$ Shockwave therapy

$\square$ Laser therapy

$\square$ Short wave diathermy

$\square$ Interferential therapy

$\square$ Orthosis

$\square$ Taping

$\square$ Bio-feedback

$\square$ Acupunkture

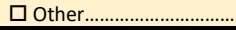

DU032

DU118

DU119

DU007

$\square$ Much better

$\square$ Quite much better

$\square$ Unchanged

$\square$ Quite much worse

$\square$ Much worse 
5. Clinical reasoning and process pathway for therapists

A thorough history and adequate physical examination are of great importance in order to target treatment interventions. In addition, it is very important to exclude the few red flag cases that require acute medical or specialist referral for the investigation and treatment of tumors, infections, inflammatory diseases, more severe back pathology and neurological conditions, as well as the strong influence of psychosocial factors which can also cause back pain. StarT Back Tool can be used to support decision making regarding the extent of health care needed and the need for psychosocial focus based on an assessment of risk factors for continued back pain. The physical assessment should include an analysis of functional movements, posture, active movements, passive movements, combined movements and / or static positions, joint accessory movement / provocation tests and neuromuscular function. This is to investigate how the symptoms are related to motion dysfunction.

Based on assessment findings, relevant treatment measures with effect mechanisms directed at functional impairments and activity limitations should be tested. These may include range of movement exercises (active/passive or accessory joint mobilisation or neuromuscular structure mobilisation), motor control exercises, muscle stretching, balance exercises, coordination, muscle strength, muscle endurance, general physical fitness or cardiovascular exercise. For example:

1. In the identification of movement directions and positions that reduce or centralize the patient's localised pain, distal pain or radiculopathy, these may be considered as a treatment technique. This allows the patient to learn strategies to control pain and thus take better responsibility for his or her own situation.

2. In the identification of movement restriction due to joint, muscle or nerve related impairment, mobilisation strategies for the relevant structure may be considered to reduce the movement restriction.

3. In the identification of segmental instability or trunk motor control impairment, exercises with a focus on movement control can be tested aiming to improve muscle function, reduce pain and optimise loading of the trunk during full body movement.

4. In the identification of a psychogenic causes of back pain, supervised exercise could be tested to minimize kinesiophobia. This can often be complemented with patient education that can help pain management and enable self-care.

5. In the identification of a postural impairment, posture correction and ergonomic interventions can be tested.

Dosage of treatment measures should be individualised and sufficient to achieve the desired effect. Initial targeted treatment should be through individual patient care. As a complement to the initial targeted treatments, the purpose of a general training and patient education is to restore or improve function and activity. The suitability of group-based patient care is assessed in consultation with the patient as general training and patient education is considered relevant to support the patient's self-care. 


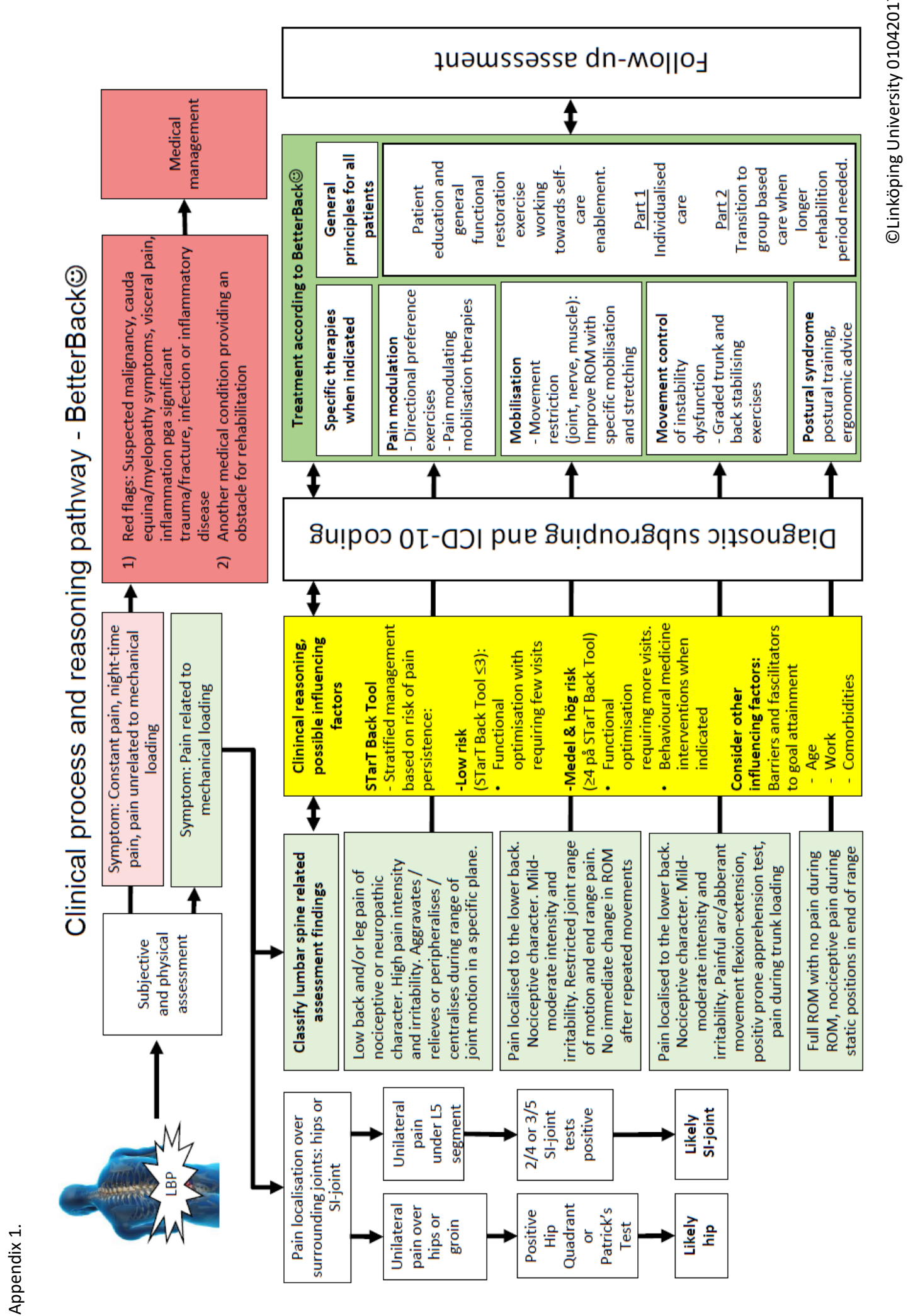


Appendix 1.

6. BetterBack $:-$ Model part 1 - Patient education brochure

\section{BetterBack:;}

\section{Information on Low Back Pain}

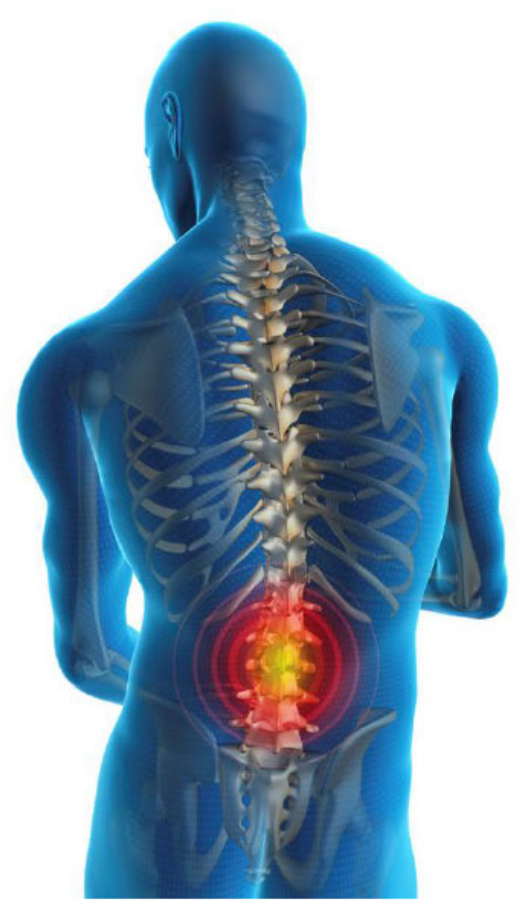

11 LINKÖPINGS 


\section{Low Back Pain}

Low back pain (LBP) is a common harmless condition that affects almost everyone at some point. Over a one-year period, 4 out of 10 adults experience LBP. It is often characterised by varying degrees of pain and discomfort that may impact on ability to perform activities. An episode of LBP usually improves within 2-6 weeks. Most have a fairly stable pattern of back health for many years, which may sometimes be interrupted by a period of LBP. This is a normal pattern and does not mean that the condition is getting worse over time.

\section{Degenerative changes in the spine}

Something that astonishes many is that there is no direct connection between degenerative changes in the spine and common LBP. This means that changes seen on X-rays, magnetic cameras and computer tomography can show pronounced age related changes or disc herniation in a completely painless person, while someone with LBP may have very little or no changes.

\section{The structure and function of the lower back and common causes of LBP}

The lower back consists of many structures such as bones, joints, discs, stabilising ligaments, nerves, as well as deep and superficial muscles. Pain sensations may potentially be signalled by one or more structures of the lower back. It is often difficult to specify exactly if and which structures signal pain sensations. How we maintain an upright position in different situations is called posture. An optimal posture means that the spine has the best conditions for good mobility with optimal distribution of body weight. Suboptimal posture, suboptimal loading of the back or even too little loading of the back can be possible contributing factors of LBP.

\section{Experience of back pain}

Pain is first experienced when interpreted in the brain. How the pain is interpreted depends on experience, thoughts, feelings and expectations. In some cases, pain may be experienced in the lower back but in the absence of pain signals from structures in the lower back. The pain system may also become hypersensitive and in some cases the pain can persist even though the original cause of the pain has resolved.

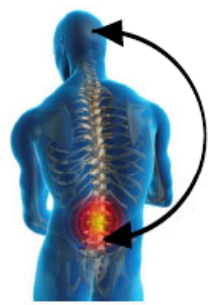

Figure 1. Pain is interpreted in the brain. This can be in the presence or absence of signals form lower back structures (C) Linköping University 20/03/2017 


\section{Back pain symptoms}

In addition to back pain, you may have pain in the buttocks and in one or both legs. You may have difficulty standing, sitting, walking, bending etc. This can lead to frustration, depressed mood and anxiety. Some may be afraid of physical activity and become inactive. All of this can impact negatively on you everyday life.

Tips when you have a particularly troublesome period

Think about what you have read in this brochure, that pain comes in periods but usually calms down. Also think about what relieves the symptoms and what you can do when you have a troublesome period. You may have a favorite exercise or other strategy to manage troublesome periods. Contact your physiotherapist for help if you feel after 2-6 weeks that pain doesn't subside. If you have numbness and tingling in both legs, loss of skin sensation or weak muscles in the legs and feet and especially if you have trouble controlling your bladder and bowel you should seek medical care. If you have LBP after an accident or have been previously treated for cancer or osteoporosis, it is also important to seek medical care. For the vast majority, however, back pain is a harmless and common condition that comes and goes.

\section{Back Health}

Good back health is a balance between the back's capacity on one side of the scale and physical / mental stresses on the other side as in the figure below.

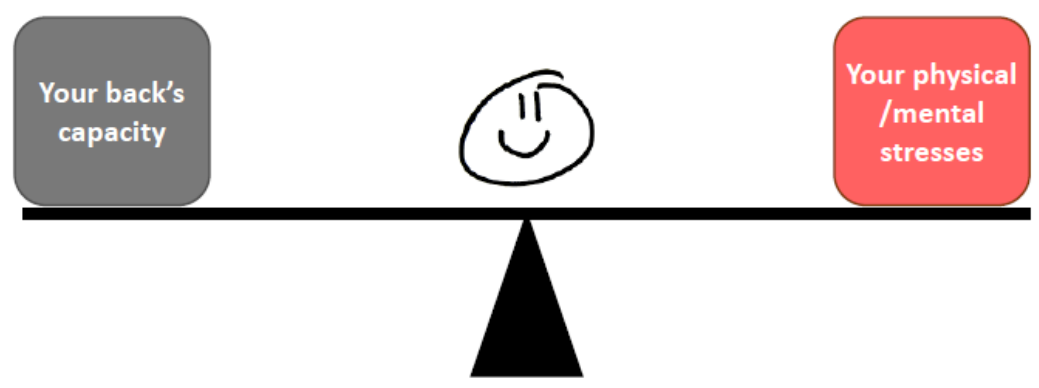

Figure 2. Balance between back capacity and stresses 
Back pain occurs when imbalance occures between back capacity compared to physical / mental stresses as in the figure below.

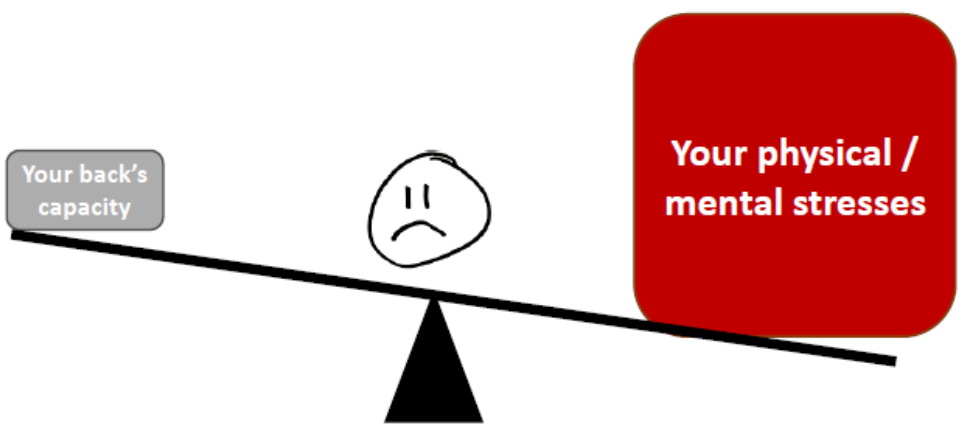

Figure 3. Imbalance between back capacity compared to physical / mental stresses

\section{General advice / self-care}

During the acute phase, most people are in need to take it easy and adjust their physical and mental stresses. Today, however, there is extensive research that recommends avoiding bedrest and instead modifying physical activity and successively returning to normal activities as quickly as possible. You can use a pain management scale to find the right level of back physical and mental stresses during everyday activities and also when you work out. This model is based on keeping you within acceptable perceived pain levels during an activity and within 24 hours after activity. This means that activity may increase the pain within acceptable pain levels during or after training, but it should return to initial levels within 24 hours. If you are unsure about the right level of back physical and mental stresses consult your physiotherapist.

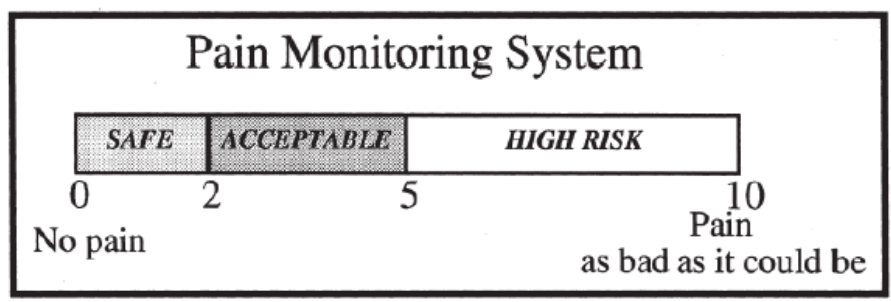

Bild 4. During activity, it is preferable that the pain is within safe to acceptable levels and that the pain returns to initial levels within 24 hours 


\section{Treatment for back pain}

The goal is to increase your back's capacity and reduce your physical and mental stresses. You can increase your back's capacity by optimising your back posture, muscle stength, muscle endurance, agility, and improving your overall fitness. You can reduce your physical and mental stresses by optimising your back's physical loads, reducing negative emotions through a positive approach and reducing everyday stress and changing your thoughts about your LBP

- Increase strength, endurance and agility

- Optimise posture

- Improve general fitness

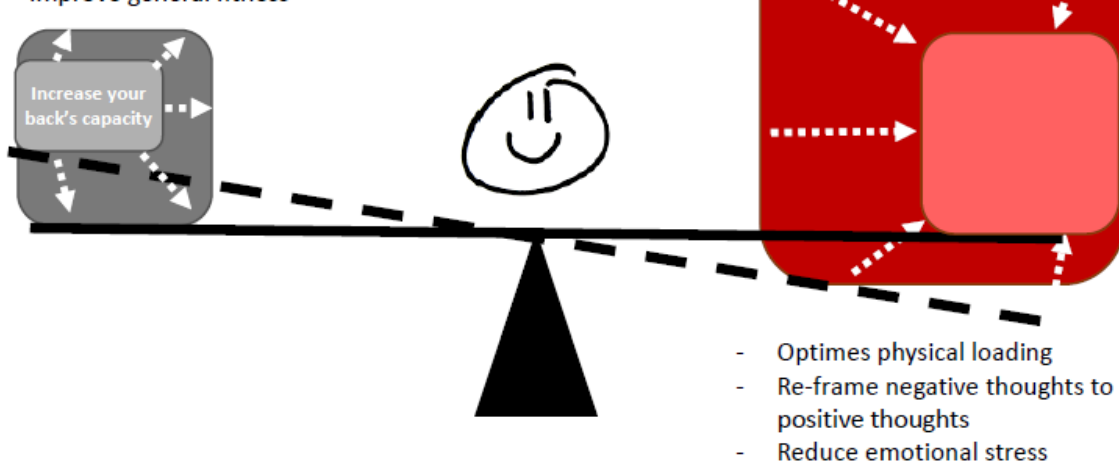

Figure 5. How to balance the back's capacity and stresses

\section{The BetterBack $(-)$ model of care}

The BetterBack $($ model of care for LBP focuses on evidence based physiotherapy, patient education and exercise. The main aim is to manage LBP symptoms and enable the patient's self-care ability. You will receive a thorough assessment and individualised care. Depending on your need for extended support in addition to your physiotherapist's initial interventions, pain education seminars and supervised exercise in a group format can be provided for 6 weeks, 2 times / week. The pain education seminars include explanatory models of what pain is, different ways of managing pain, as well as how to balance your back capacity and your physical and mental stresses you are exposed to. It is common for people to become less physically active after a troublesome period of LBP. It is therefore important to get started with some form of general fitness training. You can improve general fitness by walking, Nordic walking, cycling, jogging and swimming. If you experience pain during activity, you can use the pain management scale (see Figure 4). It is important that you feel motivated and can adapt your training to fit into your everyday life. In the BetterBack $(\cdot)$ model of care program, you can get help on how to get started!

(C) Linköping University 20/03/2017 
Appendix 1.

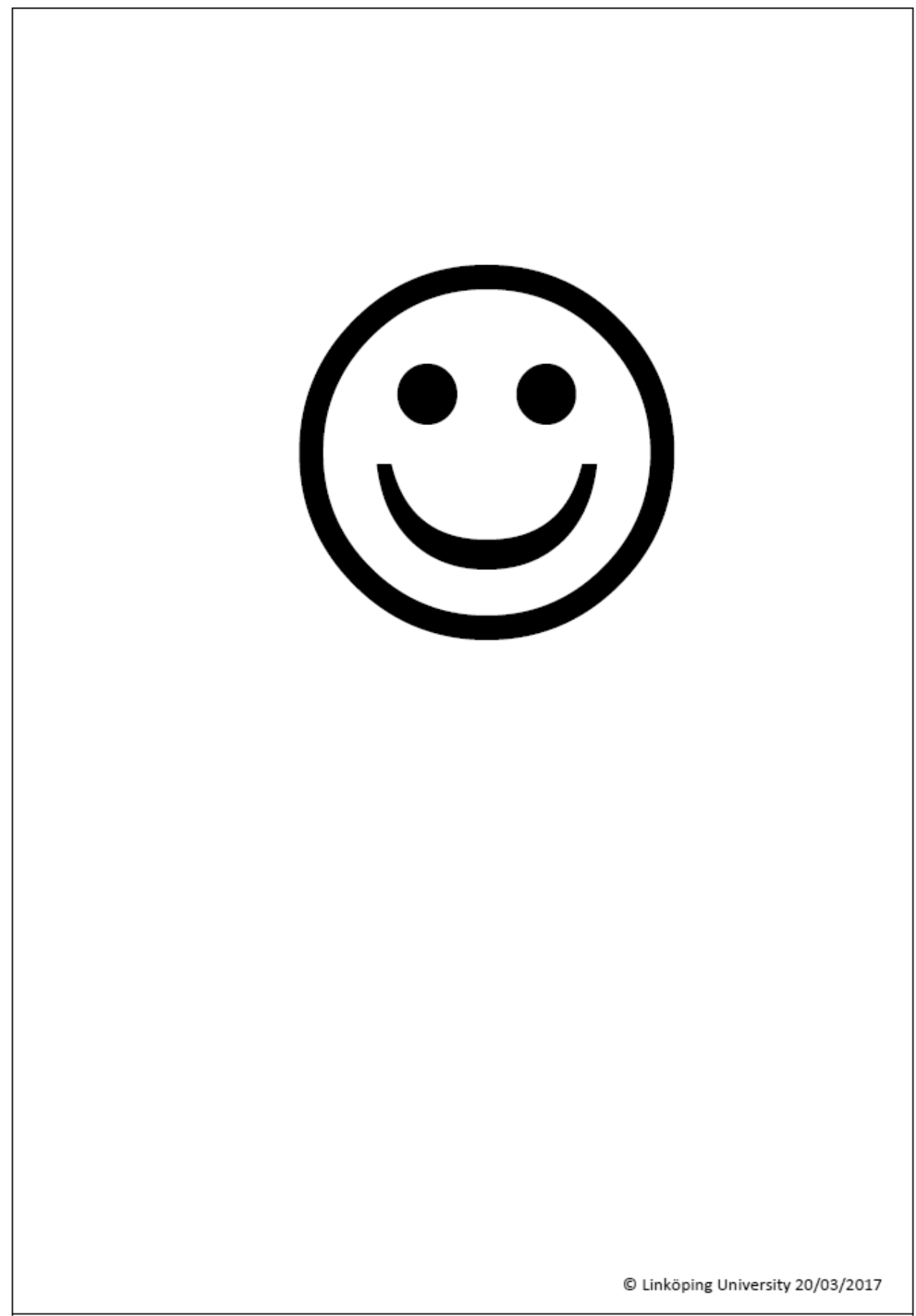


Appendix 1.

7. BetterBack $($ ) Model part 2-Group education seminar for patients
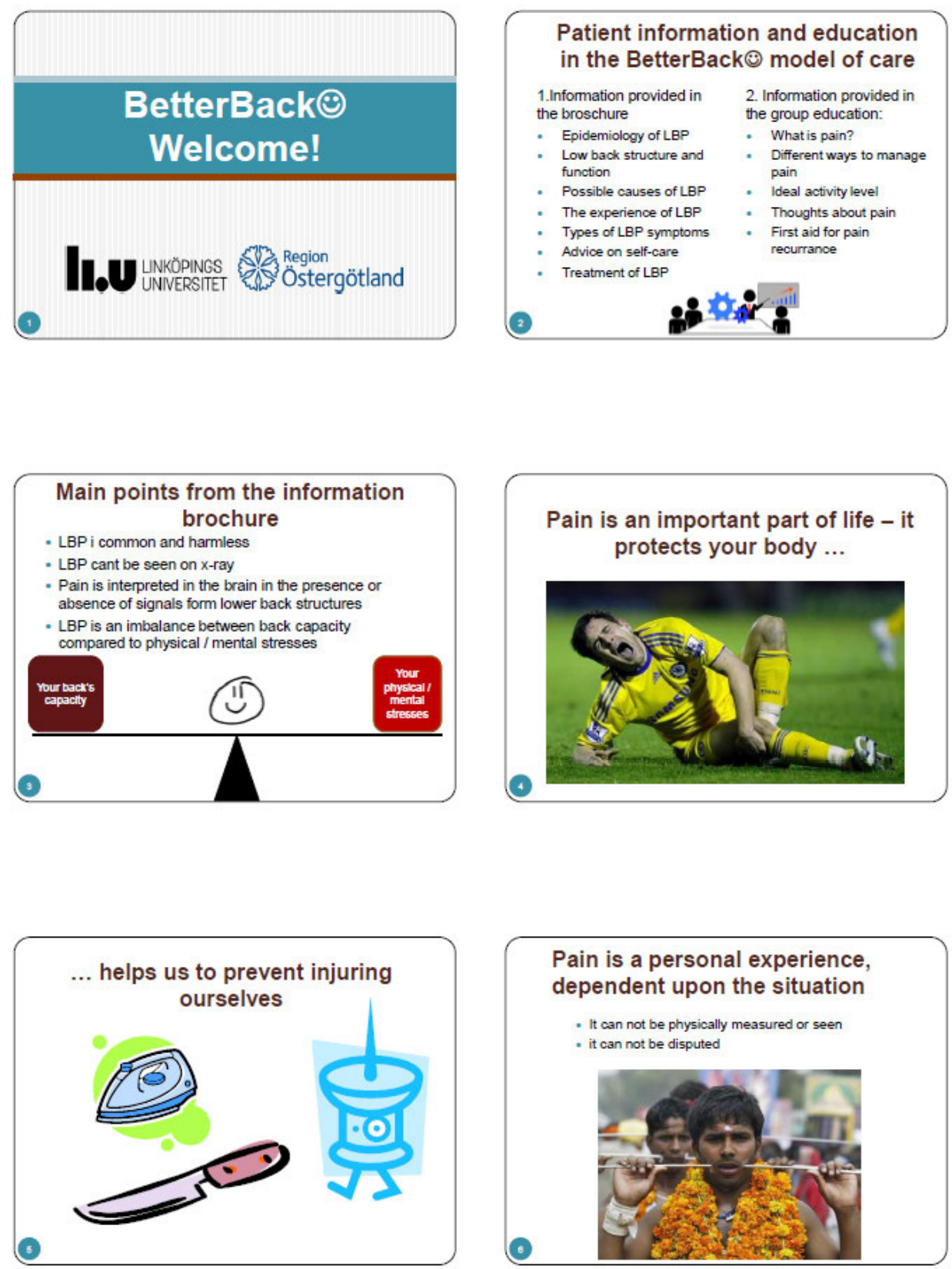

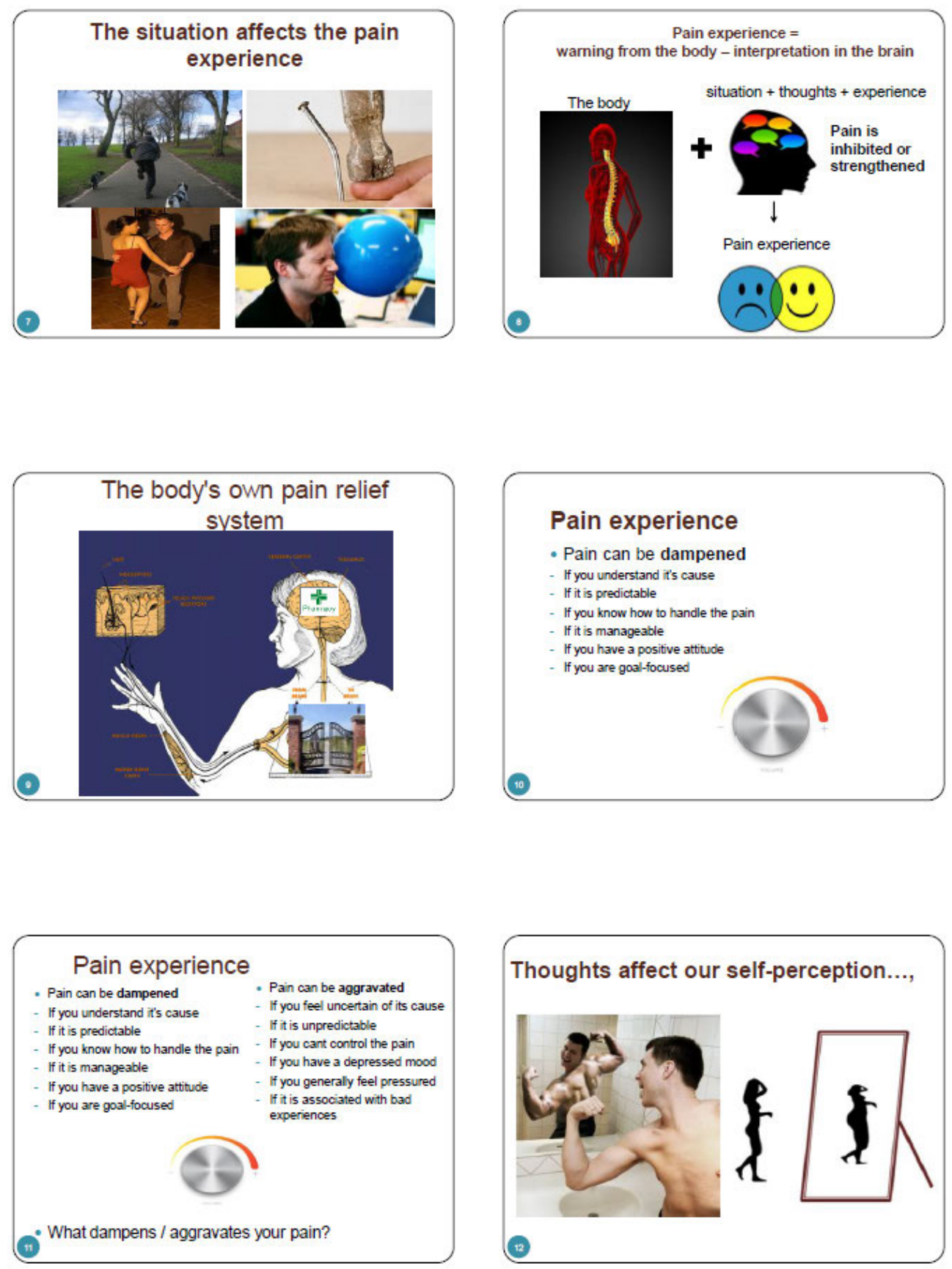

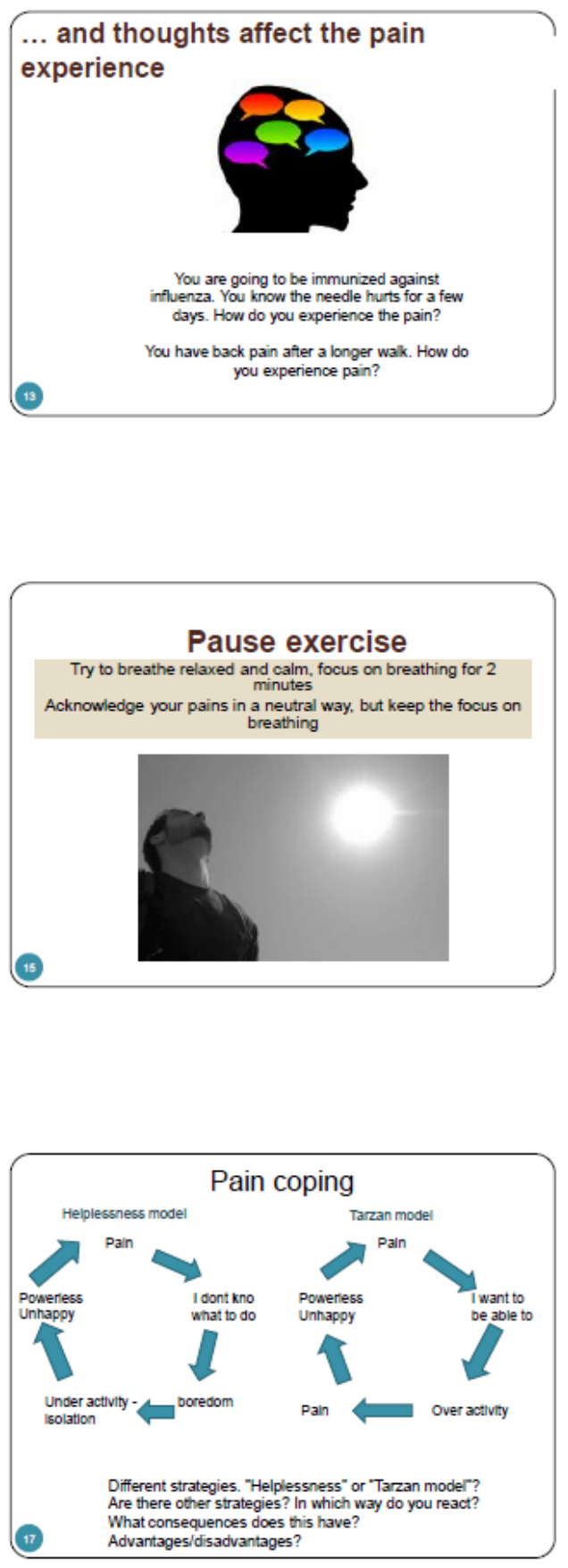

... and thoughts affect the pain experience

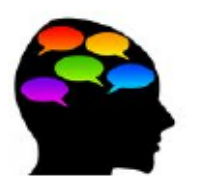

HEY MACARENA! Prolonged back pain can be Ilike a bad song that gets stuck in your mind. The brain has learned it without you wanting to and It plays over and over again... The more Irritating the song the more
easily it gats stuck in your

You started training but get more back pain afterwards. It may be muscle soreness or it may be the playing over and over again of pain memories like "the known song". How do you relate to this?

\section{Pause Exercise -redirecting}

What happens to your pain experience when you focus on your breathing?

Does this redirect your thoughts away from attention to pain?

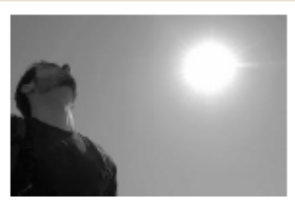

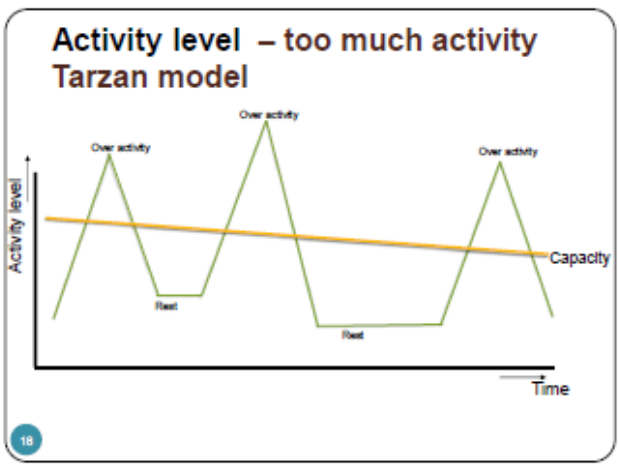



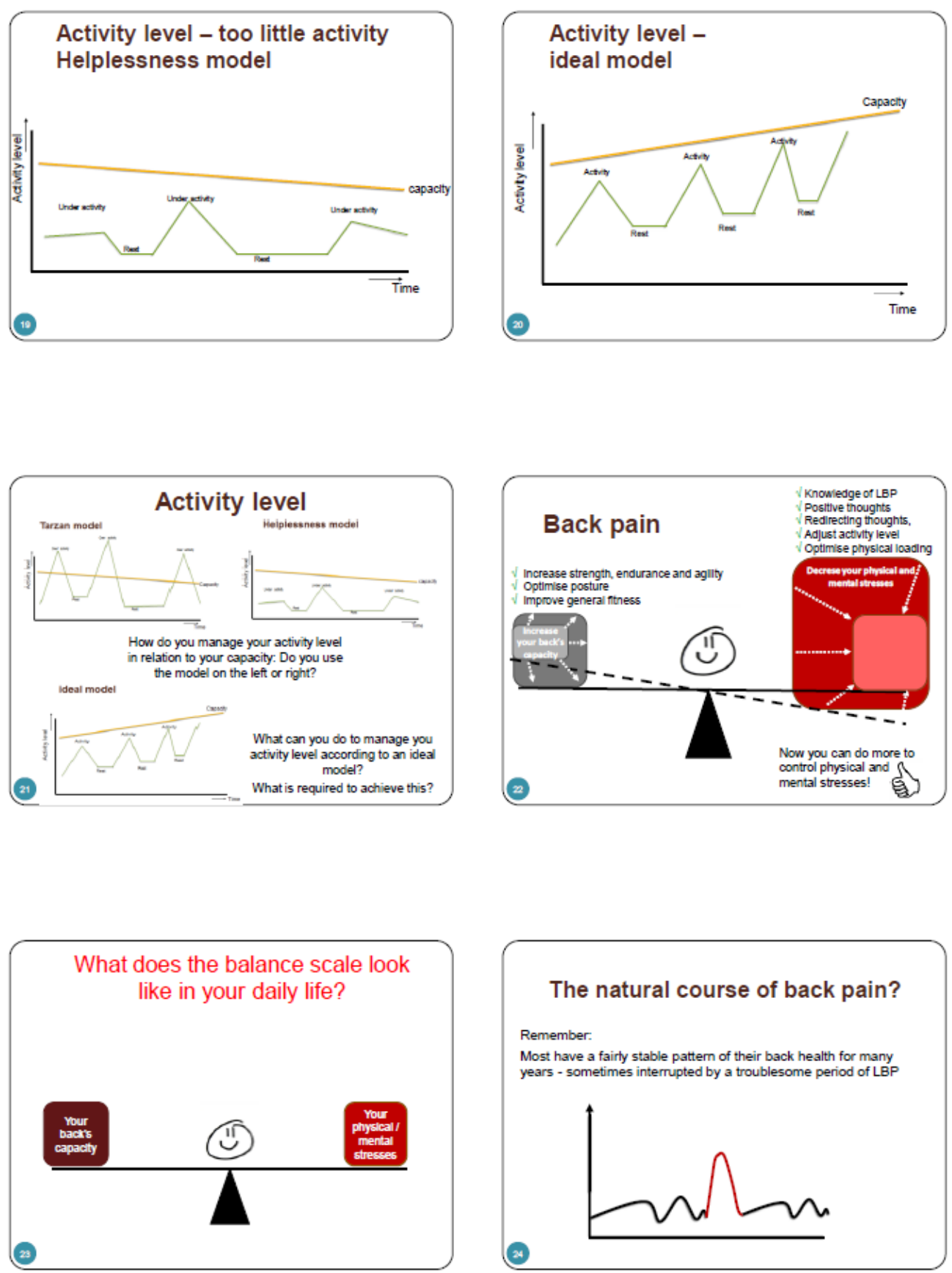

The natural course of back pain?

Remember:

Most have a fairly stable pattern of their back health for many years - sometimes interrupted by a troublesome period of LBP

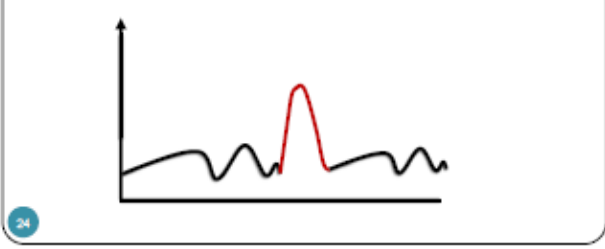




\section{First aid when back pain flares up}

- How long time do you expect the pain to be aggravated?

-What can you do when the pain gets worse?

- Do you have a favorite strategy to reduce pain?

- What can you do to make it easier for yourself?

- Ask for help?<smiles>C[14CH]=C(C)C</smiles>

pau:

Y
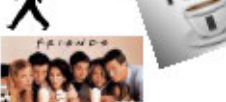

atrono

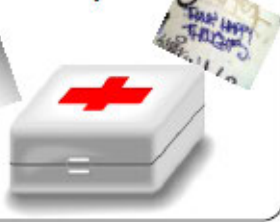

Tips...

- Adjust activity and load according to your back capacity. not too much not too ittle

- Distribute activity throughout the day

Be active, take short breaks

- Think positive thoughts

- Help youself

- And ask for help from others

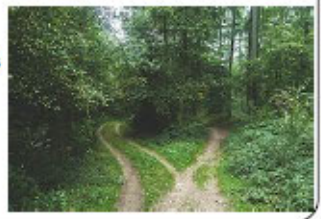

\section{Increase your capacity - improve general fitness}

Help yourself to be physically active to optimising brain and body's wellbeing.

Improved memory \& concentration

Better coping with stress

Improved mood

Divert negative thoughts

Contributes to better health,

physical and mental

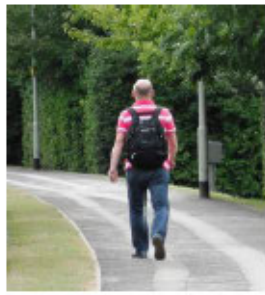

Physical activity during acute LBP
Reduce sedentary behaviour
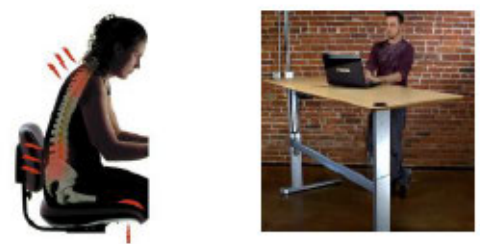

How can i change too little exercise and too much sitting?
Increase your back's capacity! We will help you get started!

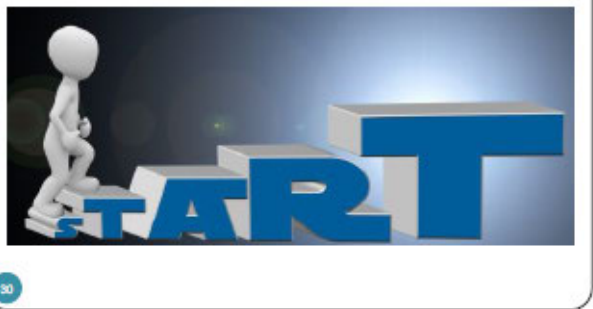


Appendix 1.

\section{Training - BetterBack()}

Supervised exercise 2 times / week for 6 weeks, then selfmediated for 6 weeks

- A little bit of training is better than none

- Remember that training can give temporary muscle soreness which is not a worsening of back pain

Your back is not fragle, the "well known pain memory song" can activate also during exercise

Talk with your physiotherapist about a long term plan after BätreRygge

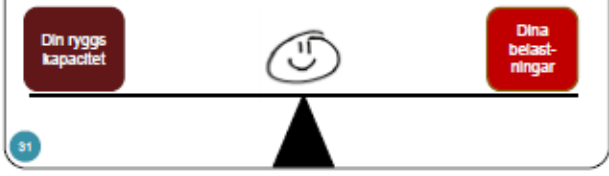

\section{Summary}

- You can use your capacity optimally

- You can redirect your thoughts

- If your back pain gets worse, do you have a plan!

- Training increases your back's capacity:

\section{Summary}

- Pain can be aggravated or dampened by many factors

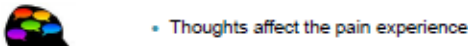

$\frac{1}{1}+$ There are different ways of coping with pain 
Appendix 1.

8. BetterBack $\odot$ Model - Training program for patients

\begin{tabular}{|c|c|c|}
\hline \multicolumn{3}{|c|}{ Training program for patients receiving the BetterBack:) model of care for LBP } \\
\hline $\begin{array}{l}\text { Part 1: Posture, } \\
\text { muscle control and } \\
\text { coordination of basic } \\
\text { body movements }\end{array}$ & $\begin{array}{l}\text { Goal: To ensure the patient has satisfactory posture and trunk muscle } \\
\text { activation in static positions as well as in conjunction with basic body } \\
\text { movement in the sitting, sitting and standing. } \\
\text { Implementation*: Exercises and dosages are individually adjusted by the } \\
\text { treating therapist. Exercises are performed as home programs and daily } \\
\text { training is recommended for optimal results. } \\
\text { The therapist assesses when basic competencies in program } 1 \text { are } \\
\text { achieved before progressing to program } 2 \text {. }\end{array}$ & $\begin{array}{l}\text { Training range of } \\
\text { movement } \\
\text { Goal: Restore normal } \\
\text { mobility. } \\
\text { Implementation: Individualise } \\
\text { based on if the patient has } \\
\text { movement restriction. }\end{array}$ \\
\hline $\begin{array}{l}\text { Part 2: Graded } \\
\text { training of muscle } \\
\text { strength, coordination } \\
\text { and endurance }\end{array}$ & $\begin{array}{l}\text { Goal: To ensure the patient has satisfactory ability to perform more } \\
\text { challenging body movements with adequate strength, coordination and } \\
\text { endurance. } \\
\text { Implementation*: Exercises and dosages are individually adjusted by the } \\
\text { treating therapist. Exercises are performed twice a week for } 12 \text { weeks } \\
\text { with follow-up conducted by the treating therapist. During the first } 6 \\
\text { weeks, patients are offered the opportunity to train in a group } \\
\text { supervised by a physiotherapist. The patient will then receive support } \\
\text { and feedback regarding the practice of exercises and help to upgrade } \\
\text { exercises if necessary. Patient education on self-care and management } \\
\text { of back pain is also performed in groups. }\end{array}$ & \\
\hline $\begin{array}{l}\text { *Prerequisite for upgrading the tr } \\
2 \text { as a basis, the physiotherapist } \\
\text { is required (in addition to a self-n } \\
\text { the patient is still the responsibil } \\
\text { where difficulty level is increased } \\
\text { produce some muscle soreness, b }\end{array}$ & 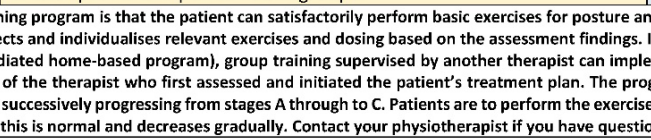 & $\begin{array}{l}\text { Ii Part 1. Using Part } \\
\text { the training program } \\
\text { ever, the follow-up of } \\
\text { ed with graded levels } \\
\text { Training can initially } \\
\text { re. }\end{array}$ \\
\hline
\end{tabular}

Part 1. Posture, muscle control and coordination of basic body movements

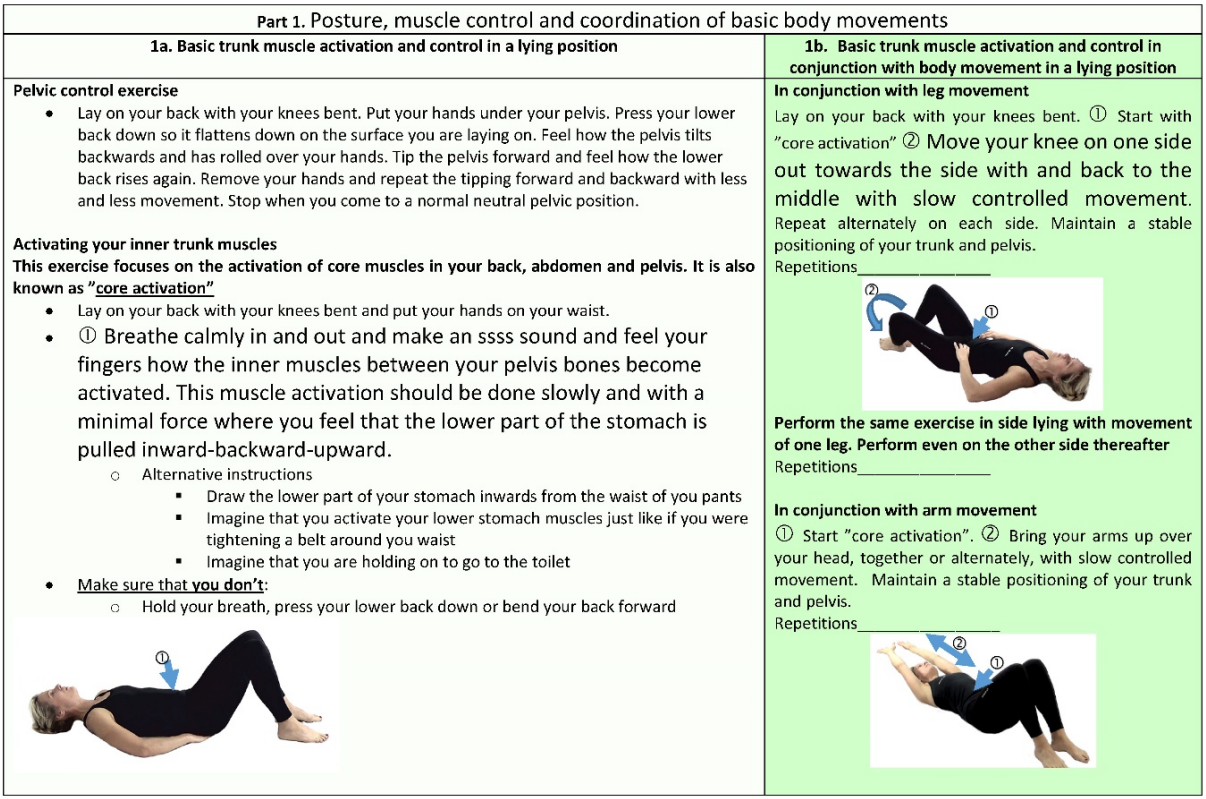


Appendix 1.

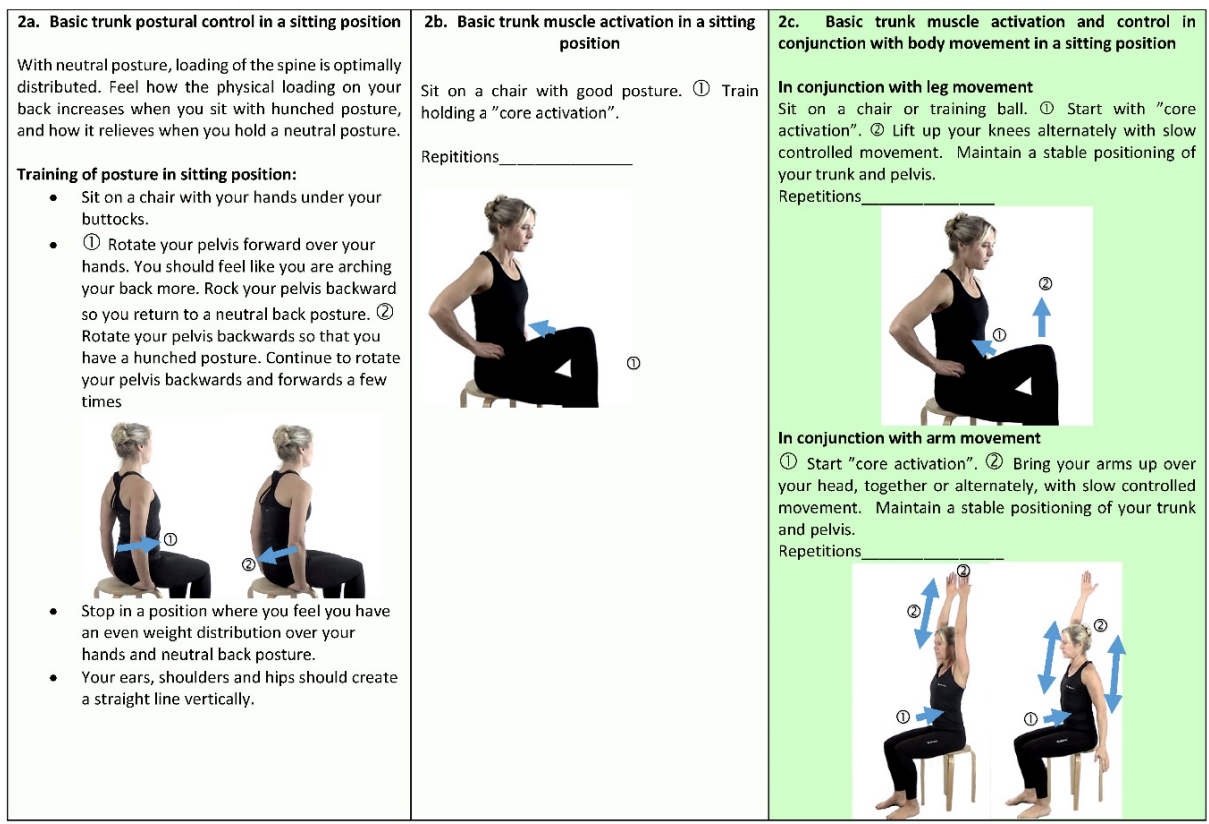

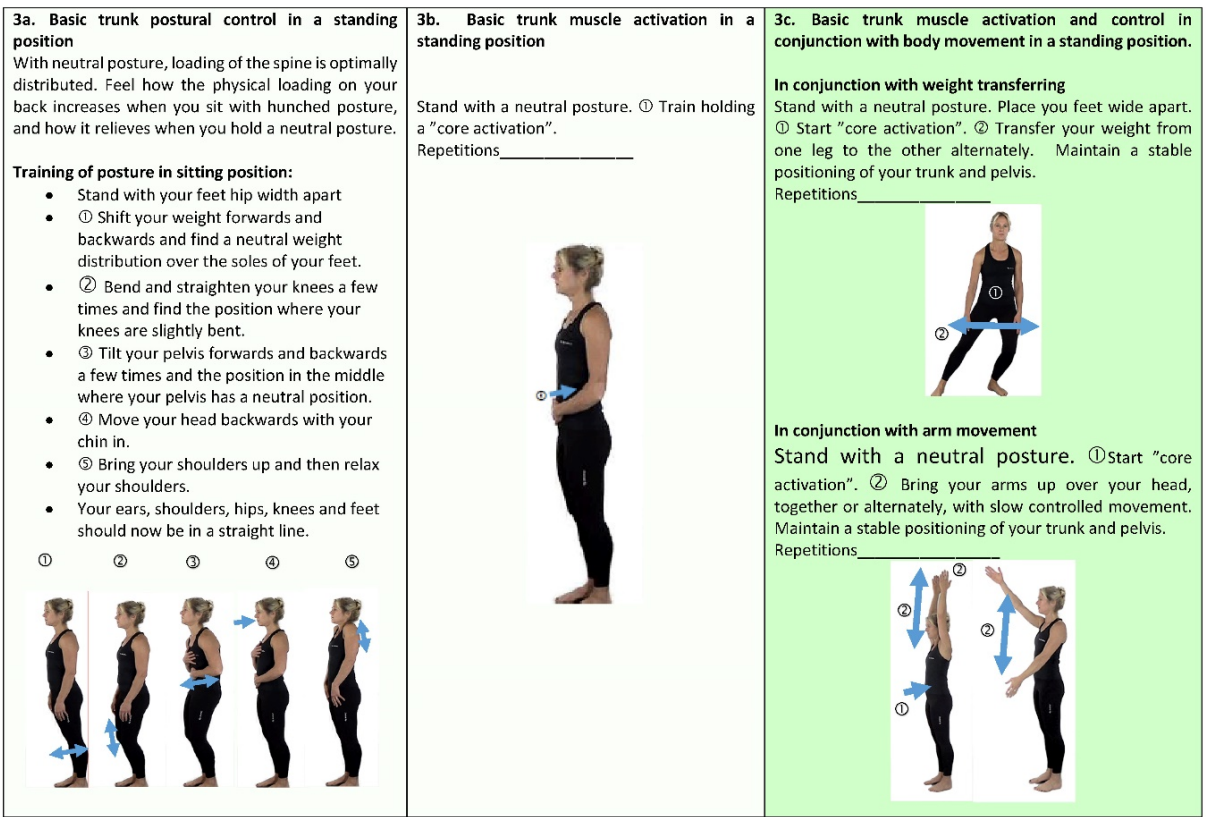


Appendix 1.

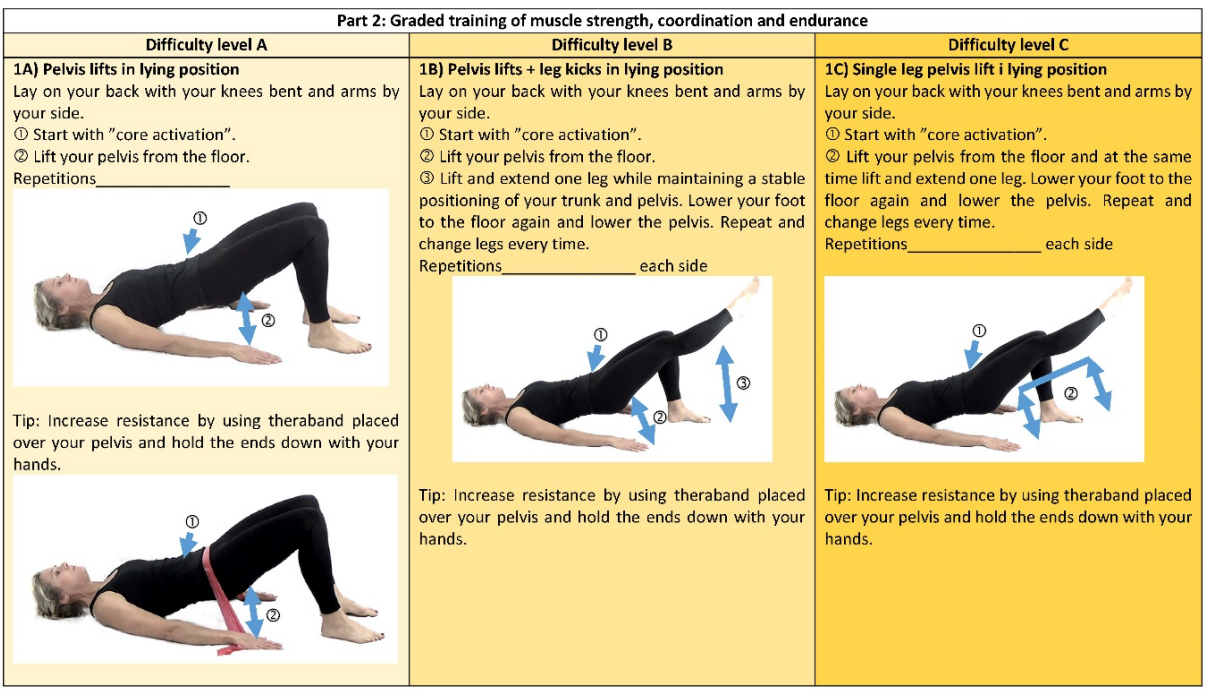

2A) Knee lifts in lying position
Lay on your back with your knees bent and put your hands on your waist.

(2) Lift one foot stowivation". maintaining a stable positioning of your trunk and pelvis. Slowly bring your foot back to the floor. Repeat and change legs every time.

Repetitions each side

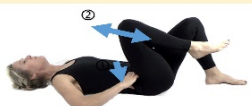

3A) Hip muscle training in lying position Lay on your back with your knees bent and arms by your side. Tie a theraband around your knees. (1) Start with "core activation".

(2) Move your knees slowly away from each other and slowly back again while maintaining a stable positioning of your trunk and pelvis.

Repetitions
2B) Straight leg raises in lying position Lay on your back with your knees bent and put your hands on your wa ist.

(1) Start with "core activation".

(2) Extend and lift one leg while maintaining a stab positioning of your trunk and pelvis. Slowly bring your leg back to the floor. Repeat and change legs every time.

Repetitions each side

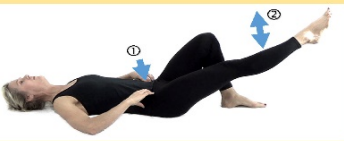

3B) Hip muscle training in side lying position Lay on your side with your knees bent. Tie theraband around your knees.

(1) Start with "core activation".

Nove your top knee slowly away from the other and slowly back down again while maintaining a

Repetitions each side
2C) Rotating sit-ups in lying position Lay on your back with your knees bent (1) Start with "core activation".

(2) Place your hands behind your head and bring your opposite knee and elbow together by bending you back forwards. Repeat alternately on each

Repetitions___ each side

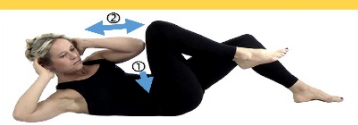

3C) Hip muscle training in side lying position Lay on your side with your legs straight. Tie a theraband around your ankles.

(1) Start with "core activation".
(2) Move your top leg slowly away from the other and slowly back down again while maintaining a

Repetitions each side 


\begin{tabular}{|c|c|c|}
\hline & & $\begin{array}{l}\text { Alternative } \\
\text { Stand on one leg in a crouched position. Straighten } \\
\text { up and move your free leg diagonally backwards } \\
\text { just like skating. Repeat alternately on each side. }\end{array}$ \\
\hline $\begin{array}{l}\text { 4A) Side plank + arm movement } \\
\text { Lay on your side with support of your lower arm and } \\
\text { knee and lift your pelvis. } \\
\text { (1) Start with "core activation". } \\
\text { (2) Maintain a stable positioning of your trunk and } \\
\text { pelvis while bringing your free arm up over your head. } \\
\text { The exercise can be done with the pelvis still (static) } \\
\text { or by moving the pelvis up and down (dynamically). } \\
\text { Perform also on the other side. } \\
\text { Repetitions }\end{array}$ & $\begin{array}{l}\text { AB) Side plank + arm movement } \\
\text { Lay on your side with support of your lower arm and } \\
\text { feet and lift your pelvis. } \\
\text { (1) Start with "core activation". } \\
\text { (2) Maintain a stable positioning of your trunk and } \\
\text { pelvis while bringing your free arm up over your head. } \\
\text { The exercise can be done with the pelvis still (static) } \\
\text { or by moving the pelvis up and down (dynamically). } \\
\text { Perform also on the other side. } \\
\text { Repetitions }\end{array}$ & $\begin{array}{l}\text { 4C) Side plank + arm movement } \\
\text { Lay on your side with support of your lower arm } \\
\text { and feet and lift your pelvis. } \\
\text { (1) Start with "core activation". } \\
\text { Q Maintain a stable positioning of your trunk and } \\
\text { pelvis while bringing your free arm up and rotating } \\
\text { your back. } \\
\text { Repetitions }\end{array}$ \\
\hline
\end{tabular}

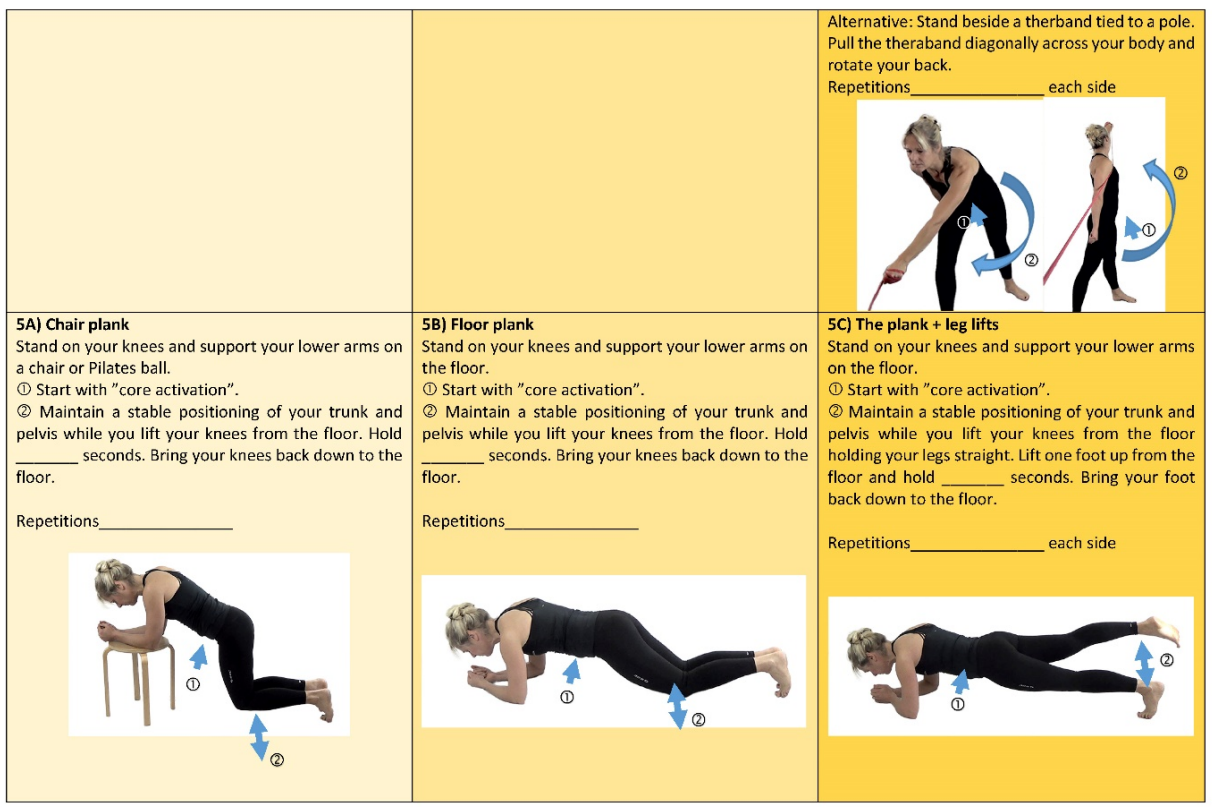


Appendix 1.

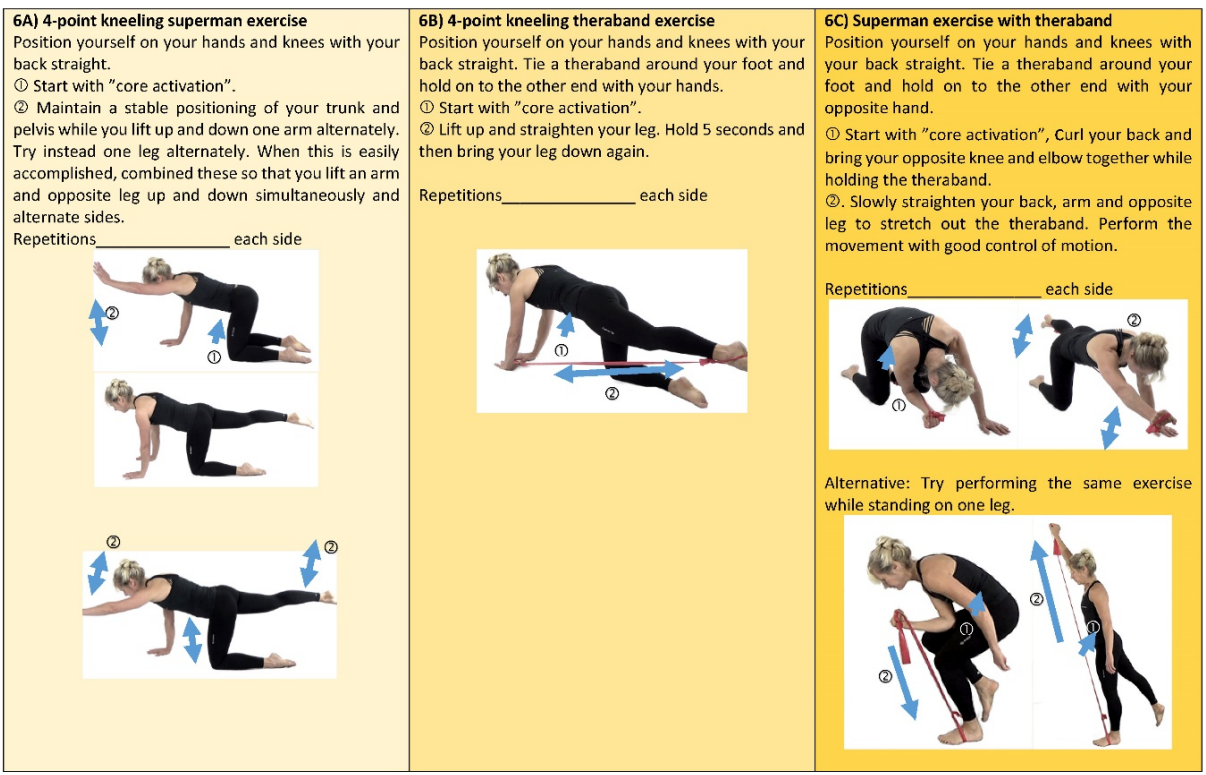

\begin{tabular}{|l|l|l|}
\hline 7A) Push-ups against a wall \\
$\begin{array}{l}\text { (1) Start with "core activation" } \\
\text { (2) Perform push-ups against a wall while maintaining } \\
\text { straight back posture. }\end{array}$ \\
$\begin{array}{l}\text { 78) Push-ups against a table } \\
\text { O Start with "core activation" } \\
\text { (2) Perform push-ups against a table while } \\
\text { maintaining straight back posture. } \\
\text { Repetitions }\end{array}$
\end{tabular}


Appendix 1.

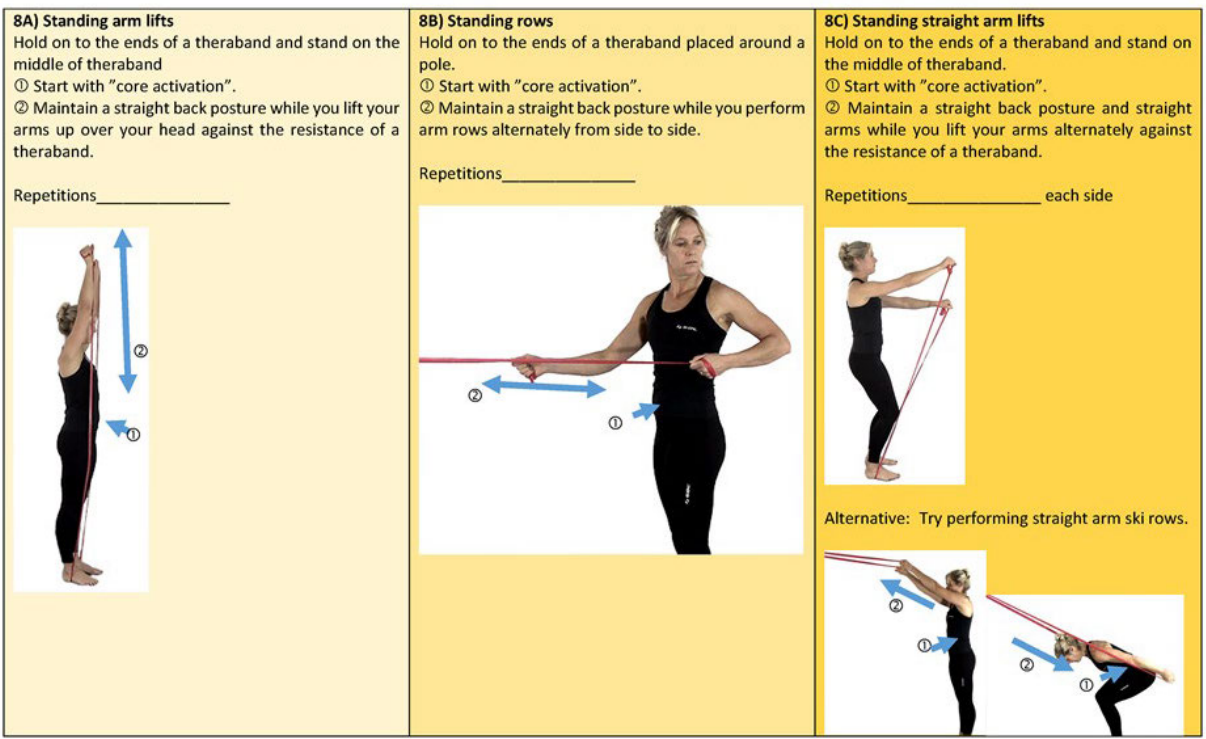

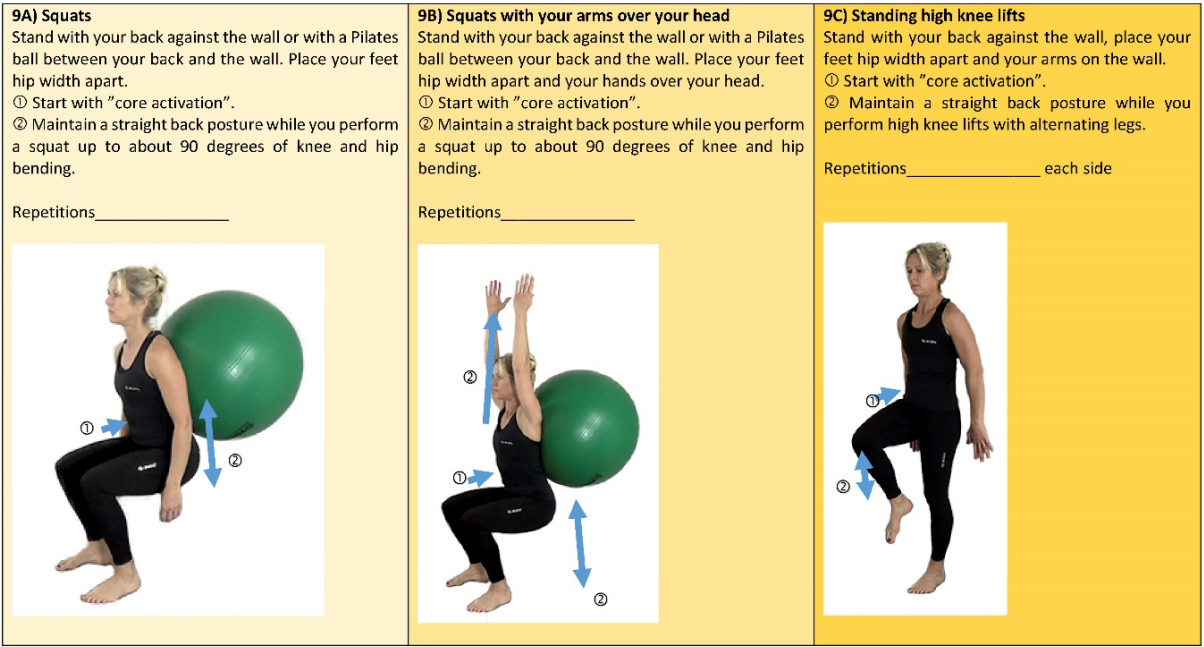


Appendix 1.

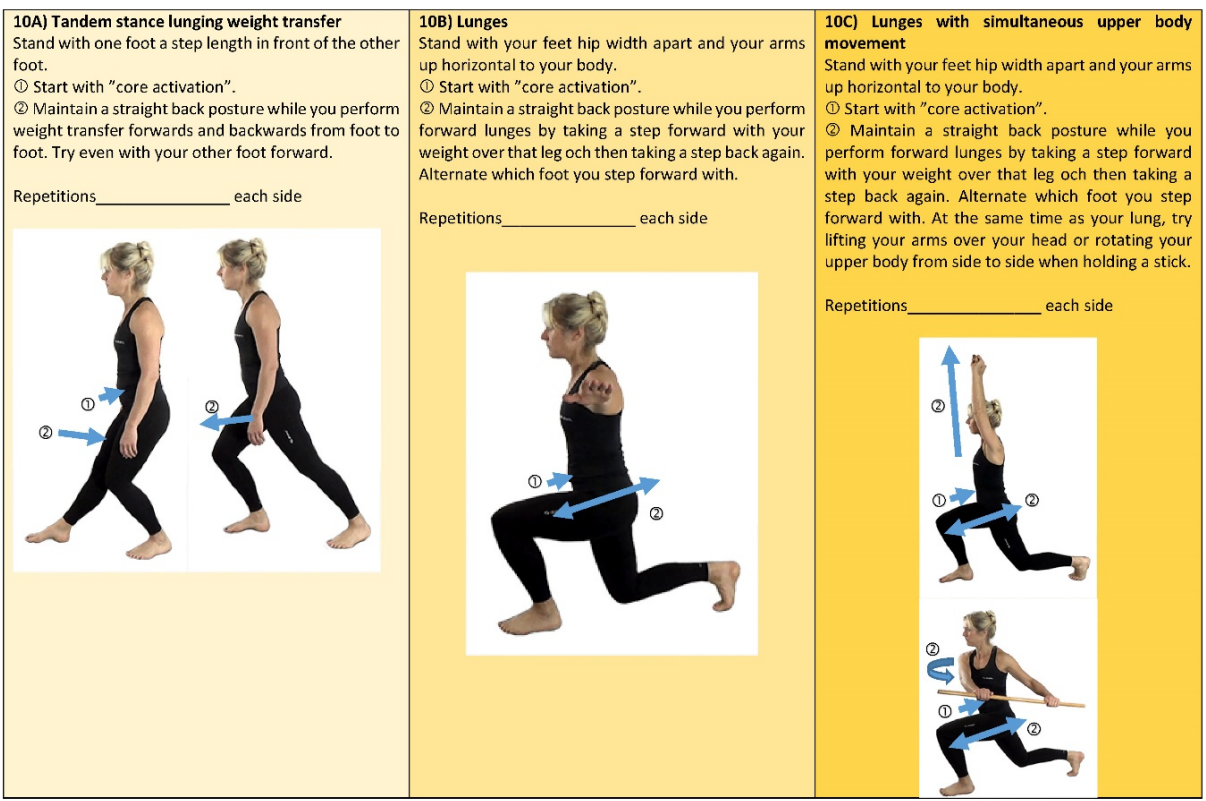

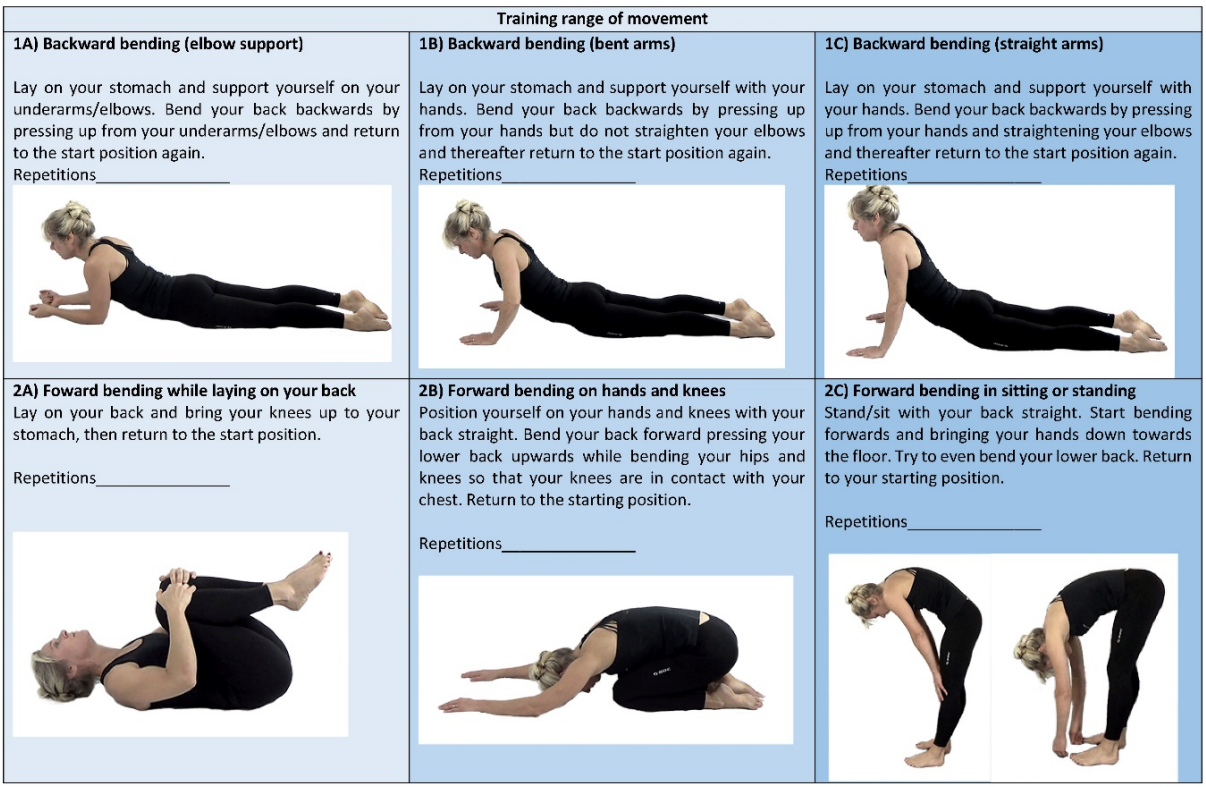




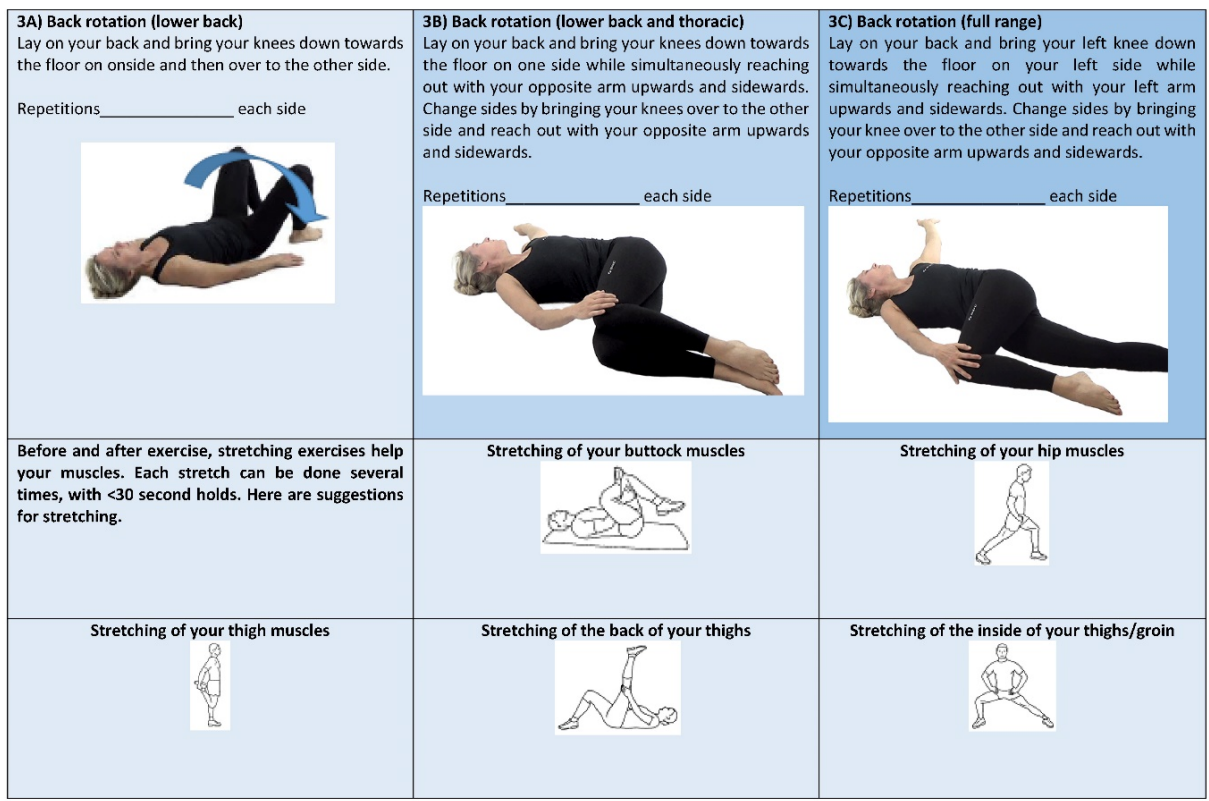

\section{General training - getting in shape}

Training form

\section{$\underline{\text { Training intensity }}$}

Training intensity can be regulated through a so-called "pacing model". This means that you slowly and gradually increase your training intensity without overloading. You "pace" yourself in a controlled way to reach your goals. You can monitor your level of exertion by using a scale of 6-20 where the scale is based on your approximate pulse when you multiply by 10 .

\section{You should preferably training with a level of exertion between}

\section{1 (fairly light) and 14 (somewhat hard).}

You should start exercising at about $20 \%$ less duration than you are capacble of. If you feel that the exercise feels very easy (at level 9 or below), you can increase your exercise duration slightly so that you feel at least a farily light exertion level (level 11). 
Appendix 1.

When you experience your exercise exertion is on average under a "somewhat hard" lavel (below 14), you can increase your exercise by $20 \%$ after 2 weeks. If you are on level 15 or more, you can continue with the same training for an additional 2 weeks.

When your training duration lasts 30 minutes, you can increase the load by increasing the intensity to $15 / 16$ (Hard - you can not speak on at this intensity) in 10-minute intervals. Then you can increase the number of minutes on this intensity (15/16) every second week.

If you have a bad day, you should work out half of what you planned. In this way you can increase your exercise gradually, without risking doing too much.

\section{Training Contract:}

I will perform .................................. as my training form
I will train 3 times/week
I will begin with .............. minutes
I will increase my training intensity with $20 \%$ every second week until
reach my goal capacity.

\begin{tabular}{l|l|l|}
\hline \multicolumn{3}{|c|}{ Rating of Perceived Exertion } \\
\multicolumn{3}{|c|}{ BOrg RPE Scale } \\
\hline 6 & Very, very light & $\begin{array}{l}\text { How you feel when lying in bed or } \\
\text { sitting in a chair relaxed. } \\
\text { Little or no effort. }\end{array}$ \\
8 & & \\
9 & Very light & \\
10 & & \\
11 & Fairly light & Target range: How you should feel \\
12 & & with exercise or activity. \\
13 & Somewhat hard & \\
14 & & \\
15 & Hard & How you felt with the hardest work \\
16 & & you have ever done. \\
17 & Very hard & Don't work this hard! \\
18 & Very, very hard &
\end{tabular}


Appendix 1.

\section{Training diary}

Name:

Your physiotherapist will fill in which exercises you should train. You can cross off when you have performed the exercises.

\begin{tabular}{|c|c|c|c|c|c|c|c|c|c|c|c|c|c|c|c|c|c|c|}
\hline \multirow[t]{2}{*}{ Week } & \multirow[t]{2}{*}{ Day } & \multicolumn{3}{|c|}{$\begin{array}{c}\text { BetterBack }: \\
\text { Part } 1\end{array}$} & \multicolumn{10}{|c|}{$\begin{array}{c}\text { BetterBack (-) } \\
\text { Part } 2\end{array}$} & \multicolumn{3}{|c|}{$\begin{array}{c}\text { BetterBack }:) \\
\text { Range of } \\
\text { movement }\end{array}$} & \multirow{2}{*}{$\begin{array}{c}\begin{array}{c}\text { General } \\
\text { training }\end{array} \\
\text { Borgskalan }\end{array}$} \\
\hline & & 1 & 2 & 3 & 1 & 2 & 3 & 4 & 5 & 6 & 7 & 8 & 9 & 10 & 1 & 2 & 3 & \\
\hline & Mon & & & & & & & & & & & & & & & & & \\
\hline & Tue & & & & & & & & & & & & & & & & & \\
\hline & Wed & & & & & & & & & & & & & & & & & \\
\hline & Thu & & & & & & & & & & & & & & & & & \\
\hline & Fri & & & & & & & & & & & & & & & & & \\
\hline & Sat & & & & & & & & & & & & & & & & & \\
\hline & Sun & & & & & & & & & & & & & & & & & \\
\hline & Mon & & & & & & & & & & & & & & & & & \\
\hline & Tue & & & & & & & & & & & & & & & & & \\
\hline & Wed & & & & & & & & & & & & & & & & & \\
\hline & Thu & & & & & & & & & & & & & & & & & \\
\hline & Fri & & & & & & & & & & & & & & & & & \\
\hline & Sat & & & & & & & & & & & & & & & & & \\
\hline & Sun & & & & & & & & & & & & & & & & & \\
\hline & Mon & & & & & & & & & & & & & & & & & \\
\hline & Tue & & & & & & & & & & & & & & & & & \\
\hline & Wed & & & & & & & & & & & & & & & & & \\
\hline & Thu & & & & & & & & & & & & & & & & & \\
\hline & Fri & & & & & & & & & & & & & & & & & \\
\hline & Sat & & & & & & & & & & & & & & & & & \\
\hline & Sun & & & & & & & & & & & & & & & & & \\
\hline & Mon & & & & & & & & & & & & & & & & & \\
\hline & Tue & & & & & & & & & & & & & & & & & \\
\hline & Wed & & & & & & & & & & & & & & & & & \\
\hline & Thu & & & & & & & & & & & & & & & & & \\
\hline & Fri & & & & & & & & & & & & & & & & & \\
\hline & Sat & & & & & & & & & & & & & & & & & \\
\hline & Sun & & & & & & & & & & & & & & & & & \\
\hline & Mon & & & & & & & & & & & & & & & & & \\
\hline & Tue & & & & & & & & & & & & & & & & & \\
\hline & Wed & & & & & & & & & & & & & & & & & \\
\hline & Thu & & & & & & & & & & & & & & & & & \\
\hline & Fri & & & & & & & & & & & & & & & & & \\
\hline & Sat & & & & & & & & & & & & & & & & & \\
\hline & Sun & & & & & & & & & & & & & & & & & \\
\hline & Mon & & & & & & & & & & & & & & & & & \\
\hline & Tue & & & & & & & & & & & & & & & & & \\
\hline & Wed & & & & & & & & & & & & & & & & & \\
\hline & Thu & & & & & & & & & & & & & & & & & \\
\hline & Fri & & & & & & & & & & & & & & & & & \\
\hline & Sat & & & & & & & & & & & & & & & & & \\
\hline & Sun & & & & & & & & & & & & & & & & & \\
\hline
\end{tabular}




\section{Papers}

The papers associated with this thesis have been removed for copyright reasons. For more details about these see:

http://urn.kb.se/resolve?urn=urn:nbn:se:liu:diva-179586 


\section{FACULTY OF MEDICINE AND HEALTH SCIENCES}

Linköping University Medical Dissertation No. 1783, 2021

Department of Health, Medicine and Caring Sciences

Linköping University

SE-581 83 Linköping, Sweden

www.liu.se

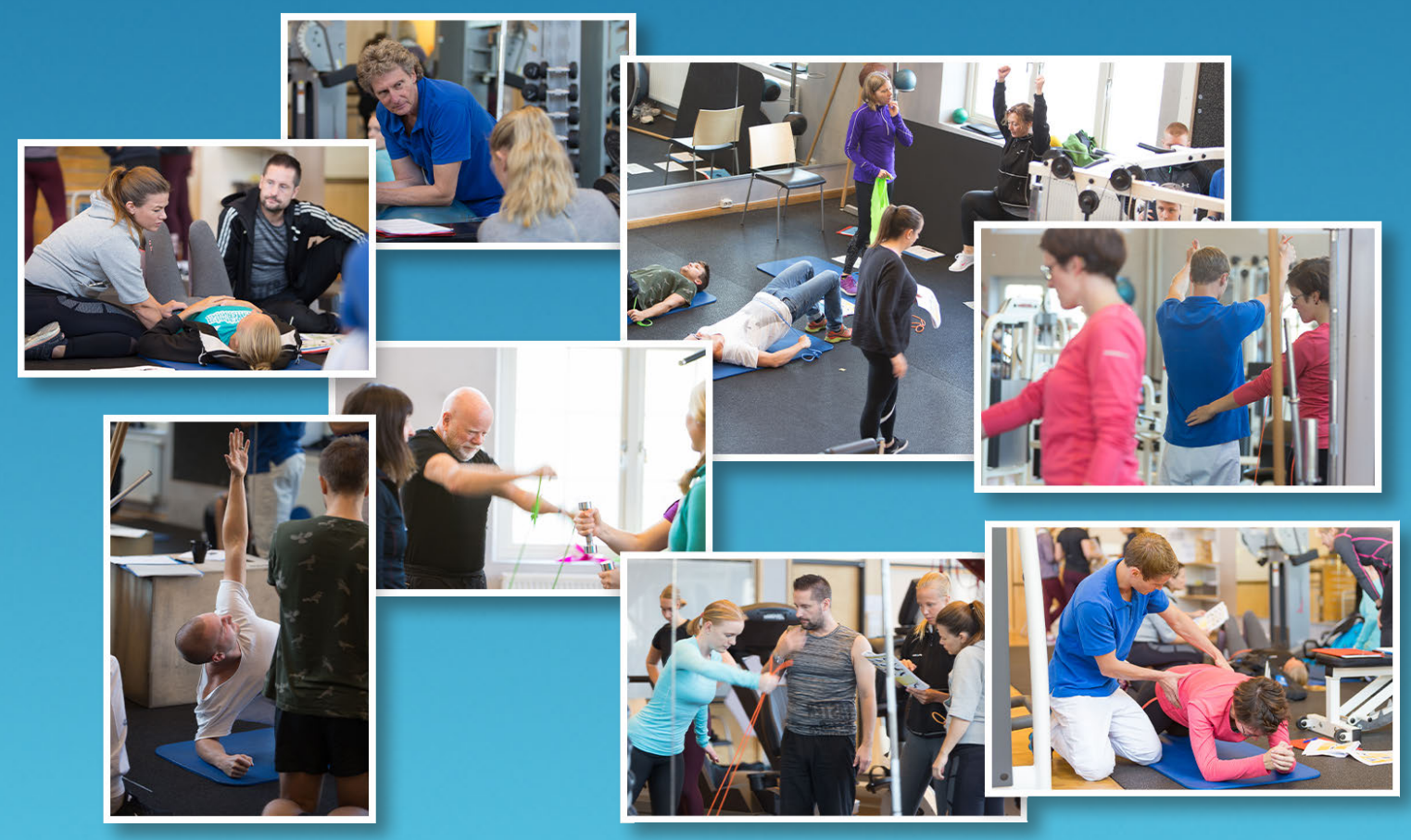

